\title{
MEDEDEELINGEN VAN DE RIJKSOPSPORING VAN DELFSTOFFEN No. 4
}

\section{BEITRÄGE ZUR KENNTNIS DER MARINEN MOLLUSKEN IM WEST:EUROPÄISCHEN PLIOCÄNBECKEN VON}

DR. ING. P. TESCH

BEZIRKSGEOLOGE FÜR NORD=LIMBURG UND NORD=BRABANT

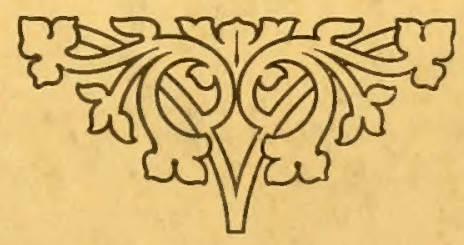

UITGEGEVEN OP LAST

VAN HET MINISTERIE VAN LANDBOUW, NIJVERHEID EN HANDEL 'S=GRAVENHAGE 1912

KOMMISSIONSVERLAG VON CRAZ UND GERLACH (JOH. STETTNER) FREIBERG IN SACHSEN 




\section{MEDEDEELINGEN VAN DE RIJKS= OPSPORING VAN DELFSTOFFEN No. 4}

MITTEILUNGEN DER STAATLICHEN BOHRVER= WALTUNG IN DEN NIEDERLANDEN

MÉMOIRES DU SERVICE DES EXPLORATIONS MINIĖRES DE L'ETAT DES PAYS=BAS

MEMOIRS OF THE GOVERNMENT INSTITUTE FOR THE GEOLOGICAL EXPLORATION OF THE NETHERLANDS

UITGEGEVEN OP LAST VAN HET MINISTERIE VAN LANDBOUW, NIJVERHEID EN HANDEL 


\section{BEITRÄGE ZUR KENNTNIS DER MARINEN MOLLUSKEN IM WEST $=E U R O P A ̈ I S C H E N$ PLIOCÄNBECKEN VON \\ DR. ING. P. TESCH}

BEZIRKSGEOLOGE FÜR NORD=LIMBURG UND NORD=BRABANT

UITGEGEVEN OP LAST

VAN HET MINISTERIE VAN LANDBOUW, NIJVERHEID EN HANDEL

's=GRAVENHAGE 1912

KOMMISSIONSVERLAG VON CRAZ UND GERLACH (JOH. STETTNER) FREIBERG IN SACHSEN 



\section{BEITRÄGE ZUR KENNTNIS DER MARINEN MOLLUSKEN IM WEST=EUROPÄISCHEN PLIOCÄNBECKEN.}

\section{EINLEITUNG.}

Unter dem Namen ,,west:europäisches Pliocänbecken” werden hier die marinen Pliocänablagerungen Englands, Belgiens und der Niederlande zusammengefasst, weil die verschiedenen Stufen in den drei Ländern aufs engste verbunden sind und offen= bar einem einheitlichen Ablagerungsbecken angehören. Von den gleichzeitigen fluvia= tilen Ablagerungen in West=Europa wird in der vorliegenden Arbeit nicht die Rede sein.

Die Fundstellen dieser pliocänen Schichten an den südöstlichen Küsten Eng= lands sind schon seit mehreren Jahrhunderten bekannt. Bereits im Jahre 1704 wurden die Fossilien in einem längst verschwundenen Aufschluss bei HARWICH von DaLE gesammelt und abgebildet; die wissenschaftliche Sammlung und Bearbeitung der reichhaltigen Conchylienfauna fängt aber erst in den ersten Decennien des vorigen Jahrhunderts an und knüpft sich vorzugsweise an die Namen James Sowerby, Samuel Woodward, Lyell und Charlesworth.

In den Jahren 1848-1850 erschien die berühmte Arbeit S. V. Woods „A Monograph of the Crag Mollusca", welche noch immer als die Grundlage der Kenntnis der englischen Pliocänfauna anerkannt wird und von F. W. Harmer als „eine der Klassiker der geologischen Literatur" bezeichnet wird. Ergänzungen zu dieser Arbeit erschienen in den Jahren 1872-1874, 1879 und 1882. Das Studium der englischen Pliocängeologie wurde von vielen anderen Geologen weiter geführt, von denen ich nur S. V. Wood Jun., Prestwich, Taylor, Alfred und Robert Bell, Kendall, Clement Reid und besonders F. W. Harmer erwähne. Die historische Entwicklung der Kenntnis dieses Gegenstandes findet man in den Arbeiten S. V. Woods und F. W. HARMERS eingehend berücksichtigt, während eine vollständige Bibliographie sich bei Clement Reid (,Pliocene Deposits of Britain”, Memoirs of the Geological Survey, 1890) findet.

Die belgischen Pliocänablagerungen im Boden der Stadt ANTwERPEN und der weiteren Umgebung wurden im Jahre 1823 zum ersten Male von Cuvier erwähnt und sind seit 1832 Gegenstand zahlreicher Untersuchungen geworden, welche durch die sich immer weiter ausbreitenden maritimen Anlagen der Stadt ermöglicht wurden. Von den vielen Forschern müssen in dieser Hinsicht besonders P. H. Nyst, P. Cogels, E. van den Broeck, M. Mourlon, J. Gosselet, hervorgehoben werden. Die Bearbeitung der Mollusken dieser Schichten von P. H. Nyst steht der Monographie S. V. Woods 
würdig zur Seite und kann mit dieser zusammen als eine feste Grundlage für die weitere Entwicklung unserer Kenntnis der west=europäischen marinen Pliocänfauna betrachtet werden. Der älteren Literatur wird in den Arbeiten E. VAN DEN BROECK's in ausführlicher Weise Rechnung getragen und sie braucht an dieser Stelle also nicht näher berücksichtigt $z u$ werden.

Die Auffindung des marinen Pliocäns im niederländischen Boden geht bis zum Jahre 1835 zurück. Für die Wasserversorgung der Stadt Gorinchem (oder Gorkum) wurde in den Jahren 1835-1837 eine $182 \mathrm{M}$. tiefe Bohrung auf dem dortigen Platz genannt "Vischmarkt" niedergebracht. Diese Bohrung wurde im Jahre 1853 von P. Harting eingehend beschrieben, wobei er eine marine Formation unter den flu= viatilen Bildungen nachwies. Die in dieser Bohrung durchteuften Schichten wurden von ihm in der nachfolgenden Weise gegliedert:

$1^{\circ}$. die "neuere alluviale Bildung" von 0.- bis $12.5 \mathrm{M}$. unter der Oberfläche.

$2^{\circ}$. die ,ältere Süsswasserbildung" von 12.5 bis 121 . - M. unter der Oberfläche. Von 74.- bis 121.- M. mit einer terrestren Molluskenfauna.

$3^{\circ}$. die "ältere Meereswasserbildung" von 121.- bis $182.4 \mathrm{M}$. unter der Ober= fläche, mit einer marinen Molluskenfauna. Diese Bildung wird von HARTing unbedingt zum Tertiär gerechnet und zwar als gleichalterig mit dem „Crag von Suffolk" betrachtet. Diese Altersbestimmung von Harting muss nach den jetzigen Auffassungen als ungefähr richtig gedeutet werden und ist 43 Jahre später von $\mathrm{F}$. W. HARMER bestätigt worden.

Die Ansicht, dass in den marinen Schichten unter Gorinchem jüngeres Tertiär vorliegt, wurde von W. C. H. Staring im Jahre 1860 bestritten. Aus der mehr oder weniger starken Abrollung der Conchylien und aus der Mischung dieser Fauna mit rheinischen Geröllen, schliesst er, dass die Fossilien auf secundärer Lagerstätte liegen. Weil die bedeckende Süsswasserbildung als „Sanddiluvium” d.h. als das jüngste Glied des Diluviums betrachtet wird und die Schichten der Süsswasserbildung und der unter= lagernden Meereswasserbildung dieselbe petrographische Zusammensetzung zeigen, glaubt er auch in dieser letzteren Bildung Ablagerungen vom Alter des jüngst=diluvialen Sanddiluviums erblicken zu müssen. Das reichliche Vorkommen von Rhein= und Maas= geröllen in fast allen Schichten bis zum Endpunkt des Bohrprofils, also in den beiden von HARTING von einander abgetrennten Bildungen, wird von STARING ausführlich erwähnt und es muss unbegreiflich erscheinen, warum er in der ,älteren Süsswas= serbildung" nicht das Aequivalent seines älteren fluviatilen Rhein= und Maasdiluviums mit Geröllen wiedererkannt hat, um so mehr, weil die Zusammensetzung der Schichten gar nicht zu seiner eigenen Beschreibung des Charakters des Sanddiluviums passt und dagegen sehr gut mit dem des Rhein= und Maasdiluviums übereinstimmt. Diese Meinung Starings wurde 25 Jahre später endgültig von LoRIÉ widerlegt, der die betreffenden Schichten zum Scaldisien stellte. 
Von P. Harring wurden schon die nachfolgenden marinen Mollusken aus der Bohrung in Gorinchem richtig bestimmt:

$\begin{array}{ll}\text { Nucula Cobboldiae } & \text { Mya arenaria } \\ \text { Leda, lanceolata } & \text { Ringicula buccinea } \\ \text { Lucina borealis } & \text { Littorina littorea } \\ \text { Cardium edule } & \text { Littorina rudis } \\ \text { Cyprina Islandica } & \text { Solen vagina } \\ \text { Corbula gibba (rotundata) } & \text { Mactra solida }\end{array}$

Die zweite Stelle, wo marines Pliocän im niederländischen Boden erbohrt wurde, ist die Stadt Goes in der Provinz Zeeland. Hier wurde in den Jahren 1864 bis 1870 eine Bohrung nach Wasser niedergebracht, welche bis zur beträchtlichen Teufe von $224 \mathrm{M}$. fortgesetzt wurde. Die allgemeinen Ergebnisse dieser Bohrung wurden von verschiedenen Personen sehr kurz erwähnt (Staring 1865, P. van Dijk 1868, A. M. K. W. van Ittersum 1869, Neyt 1875), während Dr. Bosquet die nachfolgende Liste der Conchylien von 45 bis $61 \mathrm{M}$. Tiefe veröffentlichte:

$\begin{array}{ll}\text { Natica Guillemini? } & \text { Corbula planulata (gibba) } \\ \text { Trophon gracile } & \text { Mactra deaurata } \\ \text { Purpura tetragona } & \text { Venus ovata } \\ \text { Nassa reticosa } & \text { Psammobia ferroënsis } \\ \text { Syndosmya prismatica } & \text { Cardita chamaeformis } \\ \text { Cardium decorticatum } & \text { Pectunculus variabilis } \\ \text { Cyprina Islandica } & \text { Pecten opercularis } \\ \text { Astarte Basteroti } & \text { Ostrea edulis } \\ \text { Astarte Galeotti } & \end{array}$

Im Jahre 1879 wurde die Bohrung Goes von Dr. F. SeELheim näher beschrieben und die Schichten von ihm in der nachfolgenden Weise gegliedert:

$$
\begin{aligned}
& \text { von } 0 \text { bis } 8 \mathrm{M} \text {. Alluvium } \\
& \text { 》 } 8 \text { 》5 } 45 \text { Diluvium } \\
& \text { 》45 } 71 \text { 》 Scaldisien } \\
& \text { » } 71 \text { » } 98 \text { Diestien f(Pliocän) } \\
& \text { » } 98 \text { »24 » Rupélien (Mitteloligocän) }
\end{aligned}
$$

Eine dritte Bohrung, welche die pliocänen Ablagerungen durchteufte, wurde in den Jahren 1872 bis 1876 auf dem Platz genannt „Vreeburg” in der Stadt UTRECHT niedergebracht. Nach drei und ein halbjähriger Arbeit, allerdings mit vielen Unter= brechungen, war das Loch bis $368 \mathrm{M}$. Tiefe fortgesetzt und musste bei dieser Tiefe aufgegeben werden. Über diese Bohrung bestehen mehrere kurze Mitteilungen von P. Harting (1872 und 1876), E. van den Broeck und G. Dewalque (1874 bis 1877). 
Der erste Versuch, die Ergebnisse dieser drei tiefen Wasserbohrungen in Zusam= menhang zu bearbeiten und für die Kenntnis der Verbreitung des marinen Pliocäns in unsrem Boden zu verwenden, ist von Dr. J. LoRIÉ gemacht. Im Jahre 1885 erschien seine wertvolle Monographie: , Résultats géologiques et paléontologiques des forages de puits à Utrecht, Goes et Gorkum" (Contributions à la géologie des Pays=Bas I, Archives du Musée Teyler, Série II, vol. II, troisième partie, Haarlem, 1885), welche als ein bedeutender Fortschritt in dieser Richtung bezeichnet werden muss. Die aus diesen Bohrungen stammenden Fossilien wurden von ihm alle bestimmt und die Fauna mit denjenigen der englischen und belgischen Ablagerungen verglichen. Schliesslich wird die nachfolgende Schichtengliederung aufgestellt:

Bohrung Gorinchem (Gorkum).

$$
\begin{aligned}
& \text { 0.- bis 12.- } M \text {. recente Bildungen } \\
& \text { 12.- 》 92.- 》 Diluvium } \\
& \text { 92.- 》 120.- 》 Diluvium mit Süsswasserfauna } \\
& \text { 120.- 》182.- 》Scaldisien (Pliocän) }
\end{aligned}
$$

Bohrung UTREChT.

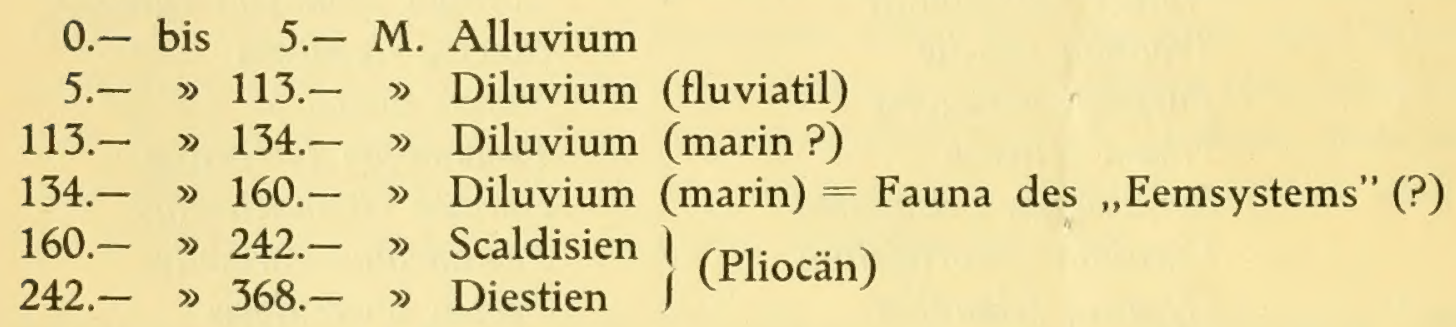

Bohrung Goes.

$$
\begin{aligned}
& \text { 0.- bis 8.- M. Alluvium } \\
& \text { 0.- 》 33.- 》 Sanddiluvium } \\
& \text { 33.- 》 62.- 》 Scaldisien । } \\
& \text { 62.- 》97.- 》Diestien f(Pliocän) } \\
& \text { 97.- 》224.- 》 Rupélien (Mitteloligocän) }
\end{aligned}
$$

Die Fossilienliste dieser drei Bohrungen führt schon 104 Molluskenarten auf, welche alle auch in den Arbeiten von P. H. Nyst und Searles V. Wood für die belgischen und englischen Ablagerungen genannt werden.

Diese Abhandlung LoRIÉs bildet die Grundlage unserer Kenntnis der nieders ländischen Pliocänfauna und hat die Aufmerksamkeit der ausländischen Geologen auf die pliocänen Ablagerungen in unserem Boden gelenkt.

Durch zwei neue Bohrungen wurde unsere Kenntnis in dieser Hinsicht be= reichert. Die erste wurde im Jahre 1887 bei Stoten, südwestlich von Amsterdam, bis zur Tiefe von $200 \mathrm{M}$. fortgesetzt, die zweite im Jahre 1888-89 bei Diemerbrug, östlich von Amsterdam, bis zur Tiefe von $335 \mathrm{M}$. Die ausführliche Beschreibung 
dieser Bohrungen gab Dr. J. LoRIÉ im Jahre 1889 in seiner Abhandlung: „Les deux derniers forages d'Amsterdam" (Contributions à la géologie des Pays:Bas IV, Bulletin de la société belge de géologie etc. 1889). Die erste Bohrung, bei Sloten, hat das marine Pliocän nicht erreicht; die zweite, bei Diemerbrug, hat von 190 bis $335 \mathrm{M}$. marine Schichten durchteufí, welche von LoRIÉ in nachfolgender Weise gegliedert werden:

$$
\begin{aligned}
& \text { 0.- bis 16.- M. Alluvium } \\
& \text { 16.- 》 24.- 》 Sanddiluvium in mariner Facies („Eemsystem") } \\
& \text { 24.- 》 65.- 》 Sanddiluvium in fluviatiler Facies } \\
& \text { 65.- 》 170.- 》 Gerölldiluvium (Rhein= und Maasdiluvium) } \\
& \text { 170.- 》 190.- 》 Scaldisien in fluviatiler Facies? } \\
& \text { 190. - 》 335.- 》 Scaldisien (marin) }
\end{aligned}
$$

Die marinen pliocänen Schichten unter Amsterdam ergaben 18 Molluskenarten, welche damals aus unserem Pliocän noch nicht bekannt waren.

Das fortgesetzte Studium des englischen Pliocäns ermöglichte es die verschie= denen Stufen der dortigen als "Red Crag” zusammengefassten Bildungen weiter zu gliedern und als mittleres Pliocän (gleichalterig mit dem belgischen Poederlien= Scaldisien) die Stufe von Walton=on=Naze, als oberes Pliocän die Stufen von New: bourn, Butley und Norwich von einander abzutrennen. So wurde es möglich diese Unterscheidung auch in den von LORIÉ als Scaldisien bezeichneten Schichten in den Niederlanden durchzuführen. Im Jahre 1896 zeigte F. W. HARMER in seiner Abhandlung "On the pliocene deposits of Holland and their relation to the english

\begin{tabular}{|c|c|c|c|c|c|c|c|c|}
\hline \multirow{3}{*}{ BOHRUNG } & \multirow{3}{*}{\multicolumn{2}{|c|}{ BEI }} & \multirow{2}{*}{\multicolumn{2}{|c|}{$\begin{array}{l}\begin{array}{c}\text { OBERES PLIOCÄN } \\
\text { (AMSTELIEN) }\end{array} \\
\text { M. U. N. N. }\end{array}$}} & \multirow{2}{*}{\multicolumn{2}{|c|}{$\begin{array}{c}\begin{array}{c}\text { MITTLERES PLIOCANN } \\
\text { (POEDERLIEN=SCALDISIEN) }\end{array} \\
\text { M. U. N. N. }\end{array}$}} & \multirow{2}{*}{\multicolumn{2}{|c|}{$\begin{array}{l}\text { UNTERES PLIOCÄN } \\
\text { (DIESTIEN) } \\
\text { M. U. N. N. }\end{array}$}} \\
\hline & & & & & & & & \\
\hline & & & von & BIS & von & BIS & von & BIS \\
\hline Goes . . & - & . & 29 & 34 & 34 & 54,5 & 54,5 & 93 \\
\hline Gorinchem . & . & . & 117,5 & 178,5 & nich & erreicht & nicht & erreicht \\
\hline Utrecht . . & . & . & 152 & 240 & 240 & 268 & 268 & 365 \\
\hline Amsterdam. & . & . & 200 & 335 & nich & erreicht & nicht & erreicht \\
\hline
\end{tabular}
and belgian crags etc." (Quart. Journal of the geological Society, London, 1896), dass die hangenden Schichten des Scaldisien LORIÉ's nicht eine mittel=pliocäne, sondern eine oberspliocäne Fauna vom Alter der ,Red Crag" von Newbourn führen. Die Gliederung der pliocänen Schichten in den vier obengenannten Bohrungen muss also nach F. W. HARMER sein: 
Die weiteren Stellen, wo LokiÉ das marine Pliocän im niederländischen Boden später noch mit mehr oder weniger Genauigkeit hat nachweisen können, sind: GRAVE, Bergen op Zoom, Breda, Arnheim, Geldersche Vallei und Walsoorden (Provinz Zeeland).

Aus diesem kurzgehaltenen historischen Ueberblick geht wohl deutlich hervor, wie lückenhaft die Kenntnis des marinen Pliocäns in unserem Boden bis vor kurzem war - eine natürliche Folge der Tatsache, dass diese Schichten fast überall nur in relativ tiefen Bohrungen erreicht werden können und ein Teil dieser Bohrungen gewiss noch unbekannt geblieben ist. Erst die Tätigkeit der staatlichen Bohrverwaltung hat diese Kenntnis wenigstens für das Gebiet Grave-Oss vervollständigt. In der Periode 19071910 wurde die Erforschung des Untergrundes des nördlichen Peelbezirkes durch die Ausführung von Flachbohrungen vorbereitet und das marine Pliocän in der Gegend Grave-Oss an vielen Stellen aufgeschlossen. Eine Vermehrung des mir jetzt zur Verfügung stehenden Materials wird voraussichtlich in nächster Zeit nicht stattfinden, weil etwaige Tiefbohrungen nur südlich der Verbreitungsgrenze dieser Schichten zur Ausführung kommen werden. Es scheint mir darum jetzt die rechte Zeit die Ergebnisse dieser Flachbohrungen und diejenigen einiger recenten tiefen Wasserbohrungen im westlichen Teil der Niederlande zu veröffentlichen und für eine Zusammenfassung der heutigen Kenntnisse unserer marinen pliocänen Ablagerungen und der darin erhaltenen Molluskenfauna zu verwerten. 


\section{ALLGEMEINE BESCHREIBUNG DER MARINEN PLIO= CÄNEN SCHICHTEN IN DEN NIEDERLANDEN.}

Der Beschreibung der marinen pliocänen Schichten in den Niederlanden kann die nachfolgende Ǵliederung des Pliocäns im west=europäischen Pliocänbecken zu Grunde gelegt werden.

\section{GLIEDERUNG DES MARINEN PLIOCÄNS IM WEST=EUROPÄISCHEN}

PLIOCÄNBECKEN (z. T. nach F. W. HARMER).

\begin{tabular}{|c|c|c|c|c|}
\hline & $\mid \begin{array}{c}\text { BEZEICH }= \\
\text { NUNG }\end{array}$ & NIEDERLANDE & BELGIEN & ENGLAND \\
\hline \multirow{2}{*}{$\begin{array}{l}\text { unteres } \\
\text { Pliocän } \\
\quad \text { I }\end{array}$} & I a & $\begin{array}{l}\text { Bohrungen in der Gegend } \\
\text { Afferden, Boxmeer, Wan= } \\
\text { rooy, Oploo u. s. w. (?) }\end{array}$ & $\begin{array}{l}\text { DiEstien z. T. } \\
\text { (sables graveleux à Hété= } \\
\text { rocètes, sables de Diest } \\
\text { et de Louvain, sables à } \\
\text { Terebratula grandis) }\end{array}$ & $\begin{array}{c}\text { Lenhamian } \\
\text { (Lenham Crag) }\end{array}$ \\
\hline & $1 \mathrm{~b}$ & $\begin{array}{l}\text { Bohrungen Goes, Wals- } \\
\text { oorden (Prov. Zeeland), } \\
\text { Beerta (Prov. Groningen) }\end{array}$ & $\begin{array}{c}\text { Diestien z. T. } \\
\text { (sables à Isocardia cor) }\end{array}$ & $\begin{array}{c}\text { Gedgravian } \\
\text { (Coralline or White Crag) }\end{array}$ \\
\hline \multirow{2}{*}{$\begin{array}{l}\text { mittleres } \\
\text { Pliocän } \\
\text { II }\end{array}$} & II a & $\begin{array}{c}\text { Bohrungen in der Gegend } \\
\text { Grave-Oss. Bohrungen } \\
\text { Utrecht, Goes }\end{array}$ & $\begin{array}{l}\text { SCALDisiEN } \\
\text { (sables à Trophon anti= } \\
\text { quum alias Neptunea } \\
\text { contraria) }\end{array}$ & $\begin{array}{l}\text { Red Crag of } \\
\text { Walton on Naze }\end{array}$ \\
\hline & II b & $\begin{array}{l}\text { Bohrungen in der Gegend } \\
\text { Grave-Oss. Bohrungen } \\
\text { Utrecht, Goes, Bergen op } \\
\text { Zoom. Sandgruben von } \\
\text { de Kauter (Prov. Zeeland). }\end{array}$ & $\begin{array}{c}\text { Poederlien } \\
\text { (sables à Corbula striata) }\end{array}$ & $\begin{array}{c}\text { Red Crag of Beau= } \\
\text { mont and Little } \\
\text { Oakley }\end{array}$ \\
\hline \multirow{2}{*}{$\begin{array}{l}\text { oberes } \\
\text { Pliocän } \\
\text { III }\end{array}$} & III a & $\begin{array}{l}\text { Bohrungen Utrecht, Goes, } \\
\text { Gorinchem, Amsterdam, } \\
\text { Breda, Oudewater, Arn } \\
\text { heim (?), Geldersche } \\
\text { Vallei (?), Geertruiden } \\
\text { berg, Barendrecht. }\end{array}$ & \multirow{2}{*}{$\begin{array}{c}\text { z. T. in mariner, z. } T \text {. in } \\
\text { fluviatiler Facies ausge= } \\
\text { bildet. } \\
\text { SoMmer du PoEDERLIEN } \\
\text { (Rutot) } \\
\text { AMSTELIEN (Stainier) } \\
\text { AMSTELO-MoséEN } \\
\text { (van Ertborn) } \\
\text { MoséEN (Mourlon) } \\
\text { ICÉNo-CROMERIEN } \\
\text { (van Ertborn) }\end{array}$} & $\begin{array}{c}\text { Newbournian } \\
\text { (Red Crag of } \\
\text { Newbourn etc.) } \\
\text { Butleyan } \\
\text { (Red Crag of Butley etc.) } \\
\end{array}$ \\
\hline & III b & $\begin{array}{c}\text { In mariner Facies in den } \\
\text { Niederlanden noch nicht } \\
\text { bekannt; wahrscheinlich } \\
\text { im nordwestlichsten Teil } \\
\text { des Landes marin ausge= } \\
\text { bildet. }\end{array}$ & & $\begin{array}{c}\text { ICENian } \\
\text { (Crag of Norwich, Chil= } \\
\text { lesford Beds, Crag of } \\
\text { Weybourne) }\end{array}$ \\
\hline
\end{tabular}




\section{A. OBERES PLIOCÄN.}

Petrographischer Charakter. Die Schichten des oberen Pliocäns in den Nieder: landen bestehen, genau wie in Belgien und England, aus einer Yrechsellagerung von feinen und sehr feinen Sanden, mittelkörnigen bis groben Sanden, welche meistens auch kleinere Gerölle führen, und mehr oder weniger sandigen Tonen. Die allgemeine Farbe der ganzen Schichtenreihe ist eine hellgraue.

Die feinen und sehr feinen Sande enthalten fast immer viel kleine Glimmer= blättchen und führen nur selten und sporadisch Conchylienreste. Dasselbe gilt auch für die Tone, welche nur ausnahmsweise in plastische, fette Tone übergehen.

Die mittelkörnigen bis groben Sande, welche mit den feinsandigen und tonigen Schichten wechsellagern, zeichnen sich besonders durch drei Eigenschaften aus und zwar durch die Führung von Geröllen, kleineren und grösseren Holzstücken und Conchylien.

Die Gerölle sind meistens nicht grösser als 6 bis 8 m.M.; solche über $10 \mathrm{~m} . M$. kommen nur vereinzelt vor. Die grosse Mehrzahl besteht aus weissem Quarz, unter= geordnet treten auch Lydit=und Hornsteinähnliche Gerölle mit Kieselringen und ganz kleine Kieseloolithe auf. Auch finden sich oft abgerollte, gelblichgrüne tonige Cons cretionen, welche viel Eisen= und Kalkcarbonat und eine Spur Ph osphorsäure enthalten und offenbar aus älteren tertiären Schichten stammen. Derartige Concretionen sind in den miocänen und oligocänen marinen Schichten weitverbreitet. Trotz der Abrollung erreichen sie in den hier in Betracht kommenden Schichten bisweilen noch ansehnliche Grösse (sogar 100 m.M. und mehr).

Die Holzstücke sind meistens nur kleine abgerollte Fragmente doch sind auch grössere Stücke nicht selten.

Die Conchylien sind meistens mehr oder weniger zerbrochen und zertrümmert, während die Sande von einem feinen Detritus, aus Muschelschalen hervorgegangen, ganz erfüllt sind. Die weitgehende Zertrümmerung der Muschelschalen durch die Brandungswellen auf dem Strande lässt sich auch jetzt beobachten. Die mehr soliden Conchylien, wie z. B. die Littorina:Gehäuse, sind öfters gut erhalten.

Palaeontologischer Charakter. Die hangenden Schichten des oberen Pliocäns, welche im allgemeinen feinsandig:tonig ausgebildet sind, führen eine terrestre Mollus: kenfauna. (Arten von Helix, Clausilia, Pupa, Succinea, Cyclostoma, Planorbis, Lymnaea, Paludina, Pisidium u. s. w.), auf welche an dieser Stelle nicht näher eingegangen wird. Die liegenden Schichten, welche die meisten gröberen Schichten umfassen, enthalten eine marine und zwar eine litorale Molluskenfauna mit vereinzelten Land:und Süss= wasserformen gemischt. Die nachfolgenden Arten können als mehr oder weniger charakteristisch für die ober=pliocänen Ablagerungen betrachtet werden, weil sie entweder gar nicht oder nur sehr selten und zweifelhaft aus dem mittlèren und unteren Pliocän bekannt sind. 
Wichtige Fossilien des oberen Pliocäns. (Amstelien):
1. Nucula Cobboldiae
2. Leda lanceolata
3. 》 myalis
4. Cardium groenlandicum
5. $\gg$ echinatum
6. Lucina (Loripes) divaricata (arcuata)
7. Mactra stultorum
8. Donax vittatus (anatina)
9. Tellina Balthica (solidula)
10. Scrobicularia (Trigonella) piperata (plana)
11. Mya arenaria
12. Littorina littorea
13. Turritella terebra
14. Chrysodomus (Trophon, Neptunea) antiquus
15. Pleurotoma (Bela) turricula Mont.

Von dieser Fauna leben bloss die unter 1, 2, 3 und 4 genannten Arten nicht mehr in der heutigen Nordsee.

Verbreitung. Die marinen oberpliocänen Schichten sind in den Niederlanden an den nachfolgenden Stellen nachgewiesen:

\begin{tabular}{|c|c|c|c|c|c|}
\hline & & \multicolumn{2}{|c|}{ M. U. N. N. } & \multirow{2}{*}{$\mid \begin{array}{c}\text { MACHTIG }= \\
\text { KEIT } \\
M .\end{array}$} & \\
\hline & & von & BIS & & \\
\hline Amsterdam . . &.$\quad$. & 200 & 335 & $\left.135^{1}\right)$ & nach HARMER und LORIÉ \\
\hline Utrecht. . . . & . . & 152 & 240 & 88 & $\gg$ \\
\hline Oudewater . . & . . & 147 & 188 & $411)$ & \\
\hline Geldersche Vallei? & ? . & $120-140$ & & & nach LORIÉ \\
\hline Gorkum . . . & . . & 117,5 & 178,5 & $611)$ & nach HARMER und LORIÉ \\
\hline Geertruidenberg & . . & $90-100$ & & & \\
\hline $\begin{array}{l}\text { Breda (und weiter } \\
\text { gebung). }\end{array}$ & Um & $25-45$ & & & \\
\hline Goes . . . . & . . & 29 & 34 & 5 & $》$ \\
\hline Barendrecht . . & . $\quad$. & 97 & 222 & 125 & \\
\hline
\end{tabular}

1) Mittleres Pliocän nicht erreicht. 
Eine marine oberpliocäne Fauna ist in Belgien bloss in einer Bohrung bei STRIJ= BEEK, 10 K.M. südlich von BREDA, in der unmittelbaren Nähe der niederländischen Grenze, aufgefunden. Hier fanden sich, zwischen 21 und 52 M. u. N. N. : Littorina littorea, Littorina rudis, Hydrobia ulvae, Mya arenaria, Lucina divaricata und Scobicu= laria piperata, nebst Arten, welche dem oberen und dem mittleren Pliocän gemeinsam sind. Alle weiteren mehr südlich gelegenen Aufschlüsse in der belgischen Campine ergaben bloss mittleres Pliocän. Die südliche Grenze der Verbreitung dieser Stufe in den Niederlanden kann also als eine $\mathrm{O} . \mathrm{W}$. Linie über Goes angenommen werden; die östliche Grenze muss etwa über Tilburg und 's Hertogenbosch nach Wageningen verlaufen, ist aber nach den heutigen Kenntnissen nicht genauer anzugeben.

Wo das Liegende dieser Schichtenreihe überhaupt bekannt ist, besteht es aus Schichten dess mittleren Pliocäns.

Gliederung. Eine weitergehende Gliederung der betreffenden Schichten ist in den Niederlanden vorläufig nur in dem Sinne möglich, dass die hangende Schichten= reihe mit terrestrer Fauna von der liegenden mit litoraler Fauna abgetrennt werden kann. Die letztere ist jedenfalls gleichalterig mit dem englischen Butleyan und New: bournian; ob die erstere genau der Icenian=stufe entspricht, lässt sich noch nicht mit Bestimmtheit feststellen.

\section{B. MITTLERES PLIOCÄN.}

Petrographischer Charakter. Die mittelpliocänen Schichten in den Nieder= landen sind im allgemeinen viel gleichmässiger zusammengesetzt als die oberpliocänen und bestehen der Hauptsache nach aus einem feinen bis mittelkörnigen Glauconit= sande (Korngrösse 0,1 bis 0,5 m.M.) mit spärlichen und zerstreuten, ganz kleinen Geröllen. Nur die hangenden Schichten sind bisweilen, wo man sich der Küste des mittelpliocänen Meeres nähert, abwechslungsreicher ausgebildet und führen auch gröbere Sandeinlagerungen und feine Kiesschnüre. Die allgemeine Farbe der ganzen mittel= pliocänen Schichtenreihe in den Niederlanden ist eine hellgrünlichgraue bis hellgrüne; die liegenden Schichten sind bisweilen durch stärkeren Glauconitgehalt etwas dunkler grün gefärbt.

Die gleichalterigen Schichten in England bestehen aus recht groben, stark oxydirten und eisenschüssigen Sanden mit viel grösseren Körnern und kleinen Ge= röllen bis $5 \mathrm{~m}$.M., während der petrographische Charakter des Mittelpliocäns in Belgien dem des niederländischen sehr ähnlich ist.

Dieser Sand enthält kleine Glauconitkörner, viel kleine weisse Glimmerblätt= chen und viel Muscheln und Muscheldetritus. Stellenweise finden sich harte Bänke, welche ausschliesslich aus verkitteten Schalentrümmern von Mollusken zusammen= gesetzt sind. Auch die Holzstücke und die abgerollten tonig=kalkigen Concretionen 
fehlen nicht. Die spärlichen Gerölle bestehen vorzugsweise aus weissem Quarz, daneben treten wieder Lydit= und Hornsteinähnliche Gerölle, kleine Kieseloolithe und ver= kieselte jurassische Versteinerungen auf. Auf den Horsten hat der obere Teil dieser Schichtenreihe offenbar längere Zeit über dem Niveau des Grundwassers gelegen : die Schichten sind an diesen Stellen rostfarbig, eisenschüssig, oxydirt und vollständig aus= gelaugt und die Conchylien sind nur als Abdrücke und Steinkerne übrig geblieben, während die widerstandsfähigen Knochen, Zähne und Lingulasschalen noch erhalten sind.

Palaeontologischer Charakter. Von palaeontologischem Gesichtspunkte sind in dieser Schichtenfolge eine obere und eine untere Abteilung zu unterscheiden, welche dem Poederlien und dem Scaldisien der Belgier entsprechen. Es fällt schwierig in den Bohrungen eine genaue Grenze fest zu stellen, weil in den Uebergangsschichten die Fossilien durch die Vorgänge des Bohrens sich vermischen. Jedenfalls sind die beiden Stufen genau in derselben Facies wie in Belgien vertreten. Sie führen eine marine und zwar eine litorale und Seichtwasserfauna; die obere Abteilung enthält jedoch auch vereinzelte terrestre und Brackwasserformen (Arten von Helix, Neritina, Melanopsis, Conovulus).

Als mehr oder weniger charakteristische Arten für die beiden Abteilungen können die nachfolgenden Mollusken betrachtet werden, welche sich entweder durch besondere Häufigkeit auszeichnen oder auch lediglich aus der betreffenden Abteilung bekannt sind.

Wichtige Fossilien der oberen Abteilung (Poederlien):

1. Cardium edule

2. 》 Parkinsoni

3. Diplodonta astartea

4. Astarte incerta

5. Mactra deaurata

6. Tellina praetenuis

7. 》 donacina

8. Mya truncata

9. Glycimeris siliqua
10. Corbulomya complanata

11. Littorina terebellata

12. \ suboperta

13. Trochus solarium

14. Natica catenöides

15. Cerithium tricinctum

16. Nassa propinqua

17. Conovulus (Melampus) pyramidalis

18. Helix nemoralis (Haesendoncki)

Wichtige Fossilien Der unteren Abteilung (Scaldisien):

1. Ostrea edulis

2. Pecten tigerinus

3. 》 Gerardi

4. 》 pusio

5. Cardita chamaeformis

6. Cardium nodosum

7. Astarte Basteroti

8. 》 Omaliusi

9. 》 obliquata
10. Astarte Burtini

11. Venus casina

12. 》 imbricata

13. Semele prismatica

14. Panopaea Faujasi

15. Vermetus intortus

16. Columbella subulata

17. Cancellaria Lajonkairi

18. Pleurotoma intorta 
In den liegenden Schichten dieser Abteilung in der Gegend Grave=Oss kom= men nicht selten obermiocäne Fossilien auf secundärer Lagerstätte vor, welche aus dem unterlagernden Obermiocän stammen.

Verbreitung. Diese Schichten sind in den Niederlanden an den nachfolgenden Stellen nachgewiesen:

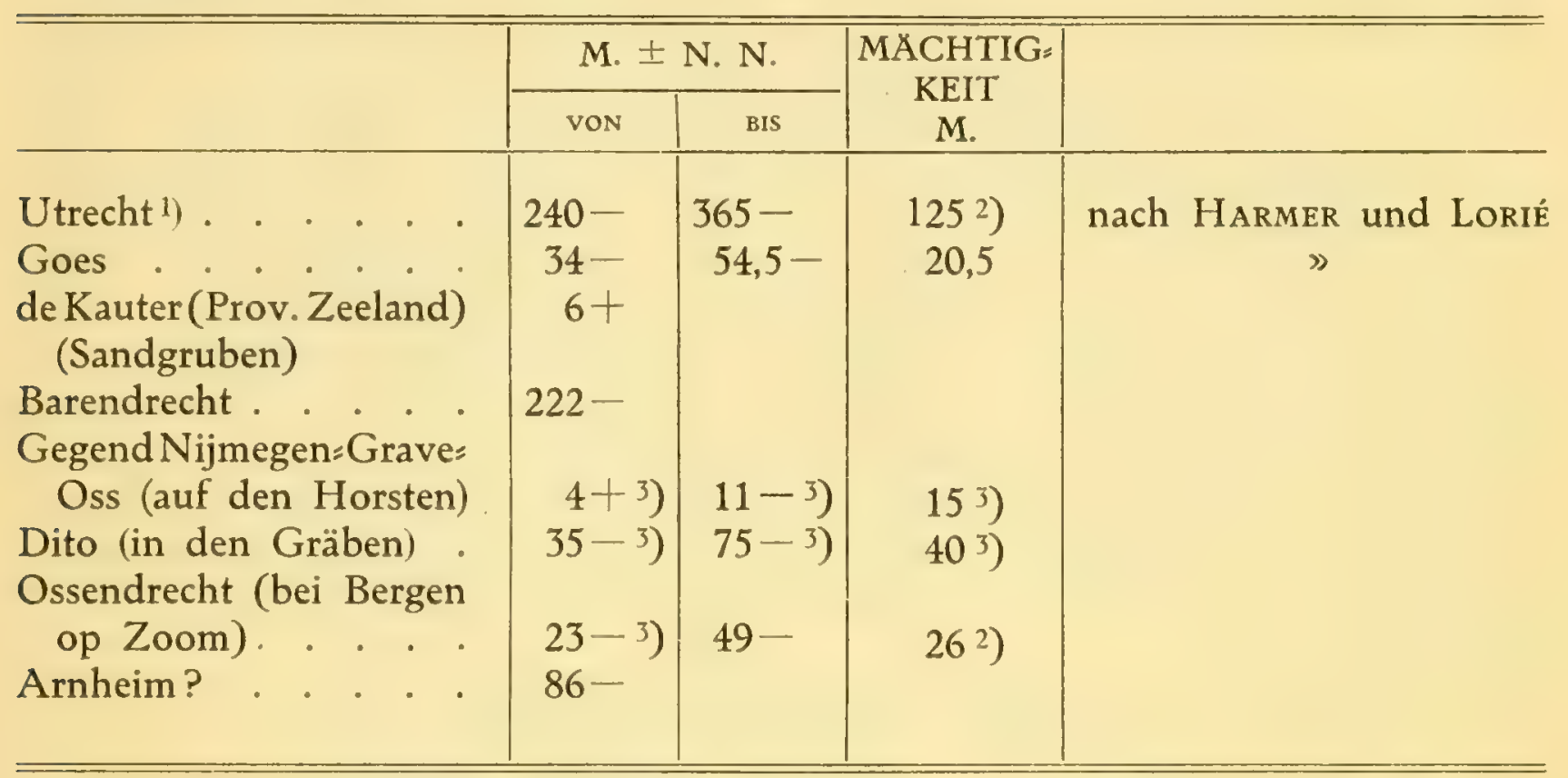

Die südliche Grenze der marinen mittelpliocänen Schichten wird im grossen und ganzen durch die Linie Vlissingen, Hulst, Hemixem (südlich von Antwerpen), Herenthals, Eindhoven angedeutet (mit einer Einbuchtung im tiefen Graben von Sittard=Roermond); die östliche Grenze ist bloss in der Gegend Grave=Oss genau bekannt und auf der beigegebenen Karte eingetragen, während sie weiter nördlich ungefähr durch die Linie CRANENBURG=MePPEL dargestellt werden muss, weil östlich dieser Linie das Fehlen des marinen Pliocäns nachgewiesen ist.

Gliederung. Wie bereits oben angeführt, sind eine jüngere (Poederlien) und eine ältere Stufe (Scaldisien) vorhanden, deren genaue Grenze in Bohrungen jedoch schwierig zu erkennen ist, um so mehr, weil der Unterschied nicht gross ist und die meisten Arten den beiden Stufen angehören. Zusammen entsprechen sie dem englischen Waltonian von $\mathrm{F}$. W. HARMER.

1) Diese Auffassung der Schichten unter Utrecht wird auf Seite 13-14 erläutert.

$\left.{ }^{2}\right)$ Liegendes nicht erreicht.

s) Mittlerer Wert. 


\section{UNTERES PLIOCAN .}

Die beiden in der Tabelle mit $\mathrm{I} a$ und $\mathrm{I} b$ bezeichneten Abteilungen des Unterpliocäns müssen jede für sich besprochen werden, weil sie sehr verschieden und die Kenntnisse in dieser Hinsicht noch sehr fragmentarisch sind.

Obere Abteilung. Es erscheint mir nicht ganz sicher ob diese Abteilung über= haupt im niederländischen Boden (die Provinz Zeeland ausgenommen) schon beob: achtet ist. Aus eigener Erfahrung kenne ich diese Stufe aus unserem Boden nicht. Sie soll damals von LoriÉ in den Bohrungen Goes, Utrecht und Grave nachgewiesen sein; diese Angaben bedürfen jedoch einer Nachprüfung.

Die meist charakteristischen Arten, welche die Abtrennung der belgischen "sables à Isocardia cor" von dem mittleren Pliocän veranlasst haben, sind die nach= folgenden:

Pecten maximus var. Westendorpi

》radians (ventilabrum)

》 similis

Lima subauriculata

Modiola sericea

Cucullaea (Arca) pectunculöides

Limopsis anomala (pygmaea)

Cryptodon (Axinus) flexuosus (sinuosus)

Cardita senilis

» orbicularis

Astarte corbulöides

Cyprina rustica (die stark angeschwollene Varietät)

Isocardia cor

Circe minima

Tellina compressa

Neaera obesa

Die Schichten von 54,5 bis 93 M. u. N. N. in der Bohrung Goes, welche von LoRIÉ dem Diestien zugerechnet wurden, ergaben eine Fauna mit Pecten radians, Pecten similis, Lima subauriculata, Limopsis anomala, Cardita senilis und Astarte corbu= löides. Viele der übrigen Arten gehören zu den Formen, welche gerade im Diestien häufiger sind als in den höheren Schichten. Ich schliesse mich also der Meinung LORIÉ's an, dass hier wirklich Unterpliocän vorliegt und führe das Auftreten ein= zelner jüngeren Formen in dieser Schichtenreihe auf Nachfall aus höheren Schichten zurück. Ablagerungen dieses Alters sind auch von LorIÉ in einer Bohrung bei WALSOORDEN in der östlichen Hälfte von Zeeuwsch=Vlaanderen aufgefunden (in einer Mächtigkeit von mindestens $12 \mathrm{M}$.), so dass die Existenz dieser Abteilung in der Provinz Zeeland als bewiesen gelten darf. 
Anders steht es nach meiner Meinung mit der Bohrung Utrecht. Die Fauna aus einer Tiefe von 268 bis 365 M. u. N. N., welche LORIÉ als Diestien betrachtet, führt als wichtige unterpliocäne Fossilien nur Pecten radians (ventilabrum), Astarte corbulöides und Tellina compressa und eine miocäne Form, offenbar auf secundärer Lagerstätte (Pecten septemradiatus). Von diesen Arten kommt Pecten radians nicht selten im unteren Scaldisien auf secundärer Lagerstätte vor, wird auch in dieser Bohrung aus mittelpliocänen und sogar aus oberpliocänen Schichten erwähnt und ist oft von einzelnen Varietäten von Pecten opercularis kaum zu trennen. Die Bestimmung von Astarte corbulöides muss, nach der Abbildung, angezweifelt werden (das Exemplar aus $311 \mathrm{M}$. Tiefe steht der Astarte Galeotti jedenfalls viel näher) und wird auch aus mittelpliocänen Schichten in dieser Bohrung angeführt, was die Zugehörigkeit dieser Exemplare zur $A$. corbulöides wohl nicht wahrscheinlicher macht. Die Bestimmung der Tellina compressa scheint mir für das abgebildete Exemplar aus $365 \mathrm{M}$. Tiefe ein= wandfrei, während das Exemplar aus 259,5 M. Tiefe, nach der Abbildung, sehr wahr= scheinlich der Tellina donacina angehört. Die übrigen Arten sprechen entschieden für mittleres Pliocän (so. z. B. Nassa elegans, Nassa reticosa, Fusus gracilis), während Nucula Cobboldiae (aus 310 - 316 M. Tiefe) in irgend einer Weise den höheren Schichten entstammen muss und dieser Fauna fremd ist. Es liegt also nur ein einziges unzweideu= tiges Exemplar einer unterpliocänen Art vor (Tellina compressa) und dieses gerade von der tiefsten Stelle des Bohrprofils. Alles zusammengenommen scheint es mir besser die ganze Schichtenreihe von 240 bis 365 M. u. N. N. noch zum Mittelpliocän $z u$ stellen. Vielleicht fängt die unterpliocäne Meeresbildung gerade hier an ; das Auf= finden der Tellina compressa in dieser Tiefe weist darauf hin.

Auch in einer Bohrung westlich von GRAvE glaubte LorIÉ in den Schichten von 5,50 bis $14,50 \mathrm{M}$. u. N. N. richtiges Diestien erblicken zu müssen; mit Aus= nahme von Pecten radians (ventilabrum) kommt in der Fauna jedoch keine einzige der wichtigen unterpliocänen Formen vor. Dagegen finden sich typische mittelpliocäne Formen wie Cardium edule, Cardium decorticatum, Tellina donacina, Nassa reticosa, Nassa labiosa, Pleurotoma costata und Cancellaria costellifera (viridula) auch in den tiefsten Schichten. Auch die Bohrungen der staatlichen Bohrverwaltung in dieser Gegend haben alle die directe Auflagerung von Mittelpliocän (Scaldisien) auf Obermiocän (Bolderien à Pectunculus pilosus) und somit das Fehlen der richtigen unterpliocänen Diestienfauna mit Isocardia cor nachgewiesen. Ich glaube also dazu berechtigt zu sein das Vorkommen dieser Abteilung des Unterpliocäns in das Gebiet Grave-Oss nicht $z u$ bestätigen.

Ich fand neulich eine Andeutung, dass diese Abteilung im östlichen Teil der Provinz Groningen existiren könnte. In der Bearbeitung der Groninger Wasserboh= rungen von Prof. Dr. F. J. P. van Calker (Beiträge zur Geologie der Provinz Groningen, Grundbohrungen, Mitteilungen aus dem mineralogisch=geologischen Institut der Reichs= Universitat zu Groningen, erster Band, II Heft, Gebr. Borntraeger, Leipzig, 1908) findet man auf Seite 139--141 eine Beschreibung einer 226 M. tiefen Bohrung bei 
BEerta. In der Tiefe 168 bis $183 \mathrm{M}$. fanden sich sporadische Muschelschalen= stückchen. Der Verfasser macht über diese Reste die nachfolgende Bemerkung : „Unter den Muschelschalenstückchen schien mir eines von einer Astarte sp., ein anderes von einer Cardita herzurühren. So liess sich leider, auch mit dem Auge auf die geringe Vertrauenswürdigkeit der Proben, nicht ausmachen, ob hier vielleicht auch in der Tiefe Tertiär erreicht ist, woran man auch wegen des oben genannten Toneisensteins denken könnte."

Im April 1911 hatte ich Gelegenheit diese Fragmente im Groninger Institut zu untersuchen und festzustellen, dass sie zweifellos zu Astarte corbulöides und Cardita orbicularis gehören. Die Entdeckung dieser beiden unterpliocänen Formen macht das Vorkommen dieser Abteilung des Unterpliocäns in dieser Gegend sehr wahrschein= lich; die Bodenproben sind leider zu schlecht um eine sichere Entscheidung möglich zu machen.

Nach dem vorher gesagten ist also die obere Abteilung in den Niederlanden bloss an den nachfolgenden Stellen aufgefunden:

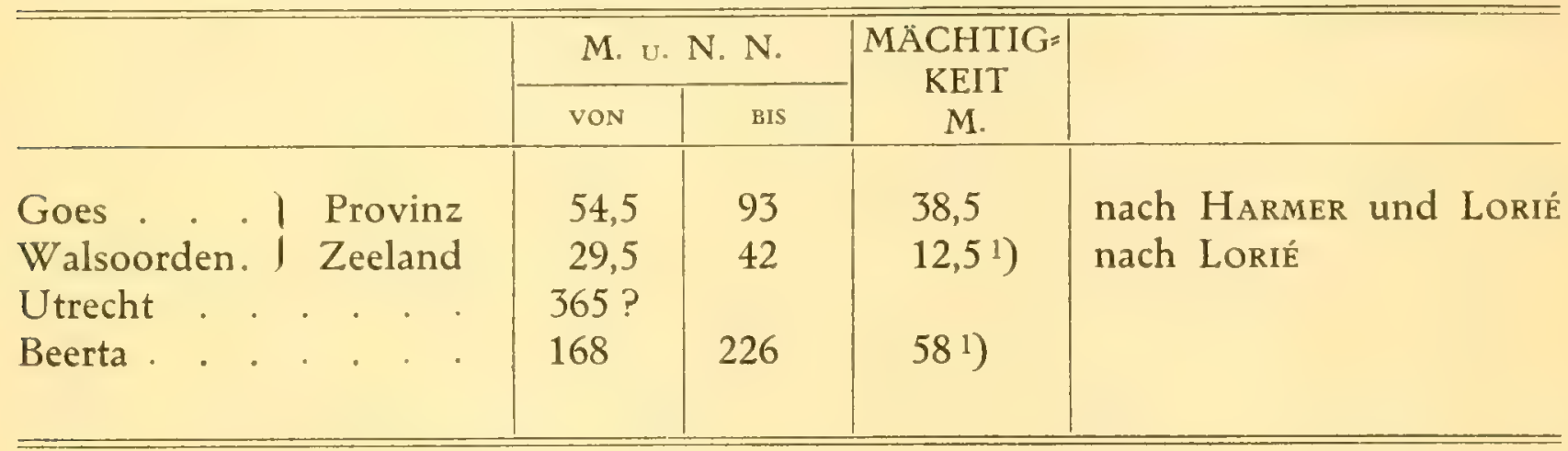

Diese Abteilung fehlt gänzlich in der Provinz Limburg und im östlichen Teil der Provinz Nord=Brabant; die östliche Verbreitungsgrenze muss jedenfalls westlich von Helmond liegen. Der petrographische Charakter ist nach den Mitteilungen LoRIÉs dem des belgischen Diestien à Isocardia cor gleich, d. h. die Schichten bestehen aus einem dunkelgrünen, feinen bis sehr feinen Glauconitsande mit Conchylien. Die Fauna ist, ge= nau wie bei Antwerpen, diejenige des offenen Meeres mit ruhigem Boden und die Schich= ten stellen also nicht die Seichtwasser= und Litoralbildungen der jüngeren Stufen dar.

Untere Abteilung. In einigen Bohrungen im nördlichen Peelbezirk (Gegend von Boxmeer, Afferden, Overloon, St. Anthonis, Wanrooy u. s. w.) findet sich über dem typischen Obermiocän (dem Bolderien à Pectunculus pilosus der Belgier) eine 1 bis 2 M. mächtige Schicht Glauconitsand mit Cetaceenknochen, Geröllen, Fisch= zähnen und Fischwirbeln und abgerollten miocänen Fossilien, welche möglicherweise der unteren Abteilung des belgischen Diestien entspricht. Sie führt massenhaft Lingula Dumortieri und Ditrupa subulata. Etwas bestimmtes lässt sich hierüber jedoch nicht sagen.

1) Liegendes nicht erreicht. 


\section{DIE MOLLUSKENFAUNA DER MARINEN PLIOCÄNEN SCHICHTEN IN DEN NIEDERLANDEN, MIT BERÜCKSICH= TIGUNG DER VERBREITUNG IN DEN GLEICHALTERIGEN ENGLISCHEN UND BELGISCHEN ABLAGERUNGEN, IN DEN DILUVIALEN SCHICHTEN UND IN DER RECENTEN FAUNA.}

BE MERK U N G E N.

1. Ich bin keineswegs der Meinung, dass alle in der nachfolgenden Liste auf= geführten Formen als „Arten”, im strengsten Sinne des Wortes, zu betrachten sind; gewisse Gruppen wären m. E. besser als Varietäten einer und derselben Art zu deuten. Aus praktischen Zwecken habe ich jedoch hier noch die übliche Unterscheidung der Arten beibehalten; eine kritische Bearbeitung dieser Formen bleibe einer späteren, rein palaeontologischen Arbeit vorbehalten.

2. Die Liste enthält nur Formen, welche in den Arbeiten von S. V. WooD und P. H. Nyst bereits abgebildet wurden. Aus diesem Grunde sind der vorliegen= den Arbeit keine Abbildungen beigegeben.

3. Die Herren F. W. Harmer (Norwich), J. J. Tesch (den Helder) und Eug. VAN DE WOUWER (Antwerpen) sage ich an dieser Stelle herzlichen Dank für ihre freundliche Unterstützung bei der Zusammenstellung der nachfolgenden Liste. Ihren hochgeschätzten Mitteilungen verdanke ich es, dass die Angaben über die Verbreitung der Formen im englischen und belgischen Pliocän und in der recenten Fauna jetzt möglichst exact sind. Die Angaben über das Vorkommen in der marinen Intergla= cialfauna („Eemien") sind der Arbeit V. Nordmanns entnommen (V. Madsen, V. Nordmann og N. Hartz. Eem=Zonerne, Studier over Cyprinaleret og andre Eem= Aflejringer i. Danmark, Nord=Tyskland og Holland. Danmarks geologiske Under= sögelse, II. Raekke, No. 17, Kjöbenhavn, 1908). 

$\mathrm{h}=$ „sehr häufig” bis „nicht selten”.

$\mathrm{S}=$,selten" bis "sehr selten".

\begin{tabular}{|c|c|c|c|c|c|c|c|c|c|c|c|c|c|c|c|}
\hline & & & & \multicolumn{12}{|c|}{ PL I OC $\mathrm{A} N$} \\
\hline & & & & \multicolumn{6}{|c|}{ NIEDERLANDE } & \multicolumn{6}{|c|}{ ENGLAND } \\
\hline & & & & \multicolumn{2}{|c|}{ I } & \multicolumn{2}{|c|}{ II } & \multicolumn{2}{|c|}{ III } & \multicolumn{2}{|c|}{ I } & \multicolumn{2}{|c|}{ II } & \multicolumn{2}{|c|}{ III } \\
\hline & & & & I & I & II & $\begin{array}{l}11 \\
\mathrm{~B}\end{array}$ & A & BII & A & I & А & 11 & III & BII \\
\hline 1 & $\begin{array}{l}\text { Pecten maximus Linn. } 1767 . \\
\quad \text { (Pecten complanatus J. Sow. 1828). }\end{array}$ & & & & & a & a & & & h & $\mathrm{h}$ & $\mathrm{h}$ & h & $\mathrm{h}$ & \\
\hline 2 & Pecten opercularis Linn. 1767 . . . & & . & & a & h & h & a & & & h & $\mathrm{h}$ & h & h & $h=s$ \\
\hline 3 & $\begin{array}{l}\text { Pecten Gerardi Nyst } 1835 . \\
\quad \text { (Pecten subdiaphanus Wood 1840). }\end{array}$ & - & . & & & $\mathrm{h}$ & $\mathbf{r}$ & $\mathrm{r}$ & & & $\mathrm{h}$ & & s & & \\
\hline 4 & $\begin{array}{l}\text { Pecten tigerinus Müller } 1776 \text {. } \\
\text { (Pecten obsoletus Penn. I777 } \\
\text { Pecten laevis Penn. } 1777 \\
\text { Pecten tigrinus Müller } 1778 \\
\text { Pecten parvus da Costa } 1778 \\
\text { Pecten domesticus Chemn. 1783). }\end{array}$ & $\cdot$ & . & & & s & & & & & h & $\mathrm{h}$ & h & h & $\mathrm{s}$ \\
\hline
\end{tabular}


$\mathrm{r}=$ auf secundärer Lagerstätte.

$\mathrm{a}=$ anwesend, jedoch unbekannt ob häufig oder selten.

\begin{tabular}{|c|c|c|c|c|c|c|c|c|c|}
\hline & & & & & & \multicolumn{3}{|c|}{ PLE I S T OCA N } & RECENT \\
\hline & \multicolumn{5}{|c|}{ BELGIEN } & \multicolumn{3}{|c|}{$\begin{array}{l}\text { FAUNA DES EEMIEN } \\
\text { (LETZTES INTERGLACIAL) }\end{array}$} & \multirow{3}{*}{$\begin{array}{c}\text { VORKOMMEN } \\
\text { IN DER } \\
\text { HEUTIGEN FAUNA }\end{array}$} \\
\hline \multicolumn{2}{|c|}{ I } & \multicolumn{2}{|c|}{ II } & \multicolumn{2}{|c|}{ III } & \multirow{2}{*}{$\begin{array}{l}\text { NIEDER } \\
\text { LANDE }\end{array}$} & \multirow{2}{*}{$\begin{array}{c}\text { NORD= } \\
\text { DEUTSCH }= \\
\text { LAND }\end{array}$} & \multirow{2}{*}{$\begin{array}{l}\text { DÄNE }= \\
\text { MARK }\end{array}$} & \\
\hline I & $\begin{array}{l}\text { I } \\
\text { B }\end{array}$ & $\begin{array}{l}\text { II } \\
\text { A }\end{array}$ & $\begin{array}{l}\text { II } \\
\text { B }\end{array}$ & III & $\begin{array}{l}\text { III } \\
\text { B }\end{array}$ & & & & \\
\hline a & $\mathrm{h}$ & $\mathrm{h}$ & $\mathrm{h}$ & $a$ & & & - & a & $\begin{array}{l}\text { Südküste Norwegens } \\
\text { (südlich von Christians } \\
\text { sund), Shetland=Inseln, an } \\
\text { der englischen Küste an } \\
\text { verschiedenen Stellen nicht } \\
\text { selten und stellenweise } \\
\text { häufig, Helgoland (nur } \\
\text { leere Schalen), den Helder } \\
\text { (im Sept. } 1908 \text { ein leben= } \\
\text { diges Ex.), Mittelländi= } \\
\text { sches Meer (spänische } \\
\text { Küste). Im allgemeinen } \\
\text { mehr südlich verbreitet. } \\
\text { In Tiefen von } 8 \text { bis } 120 \text { M. } \\
\text { An der norwegischen } \\
\text { Küste (nördlich bis Trom= } \\
\text { sö), Nordsee, an der eng= } \\
\text { lischen Küste überall an } \\
\text { sandigen Stellen häufig } \\
\text { (bisweilen in Bänken), } \\
\text { Helgoland (lebend), Ma= } \\
\text { deira, Mittelländisches } \\
\text { Meer. Diese Art ist vor= } \\
\text { wiegend litoral. In Tiefen } \\
\text { von } 5 \text { bis } 150 \text { M., sel= } \\
\text { ten in grösseren Tiefen. }\end{array}$ \\
\hline a & h & s & $\mathrm{r}$ & & & & & & $\begin{array}{l}\text { Island, norwegische und } \\
\text { schwedische Küste bis } \\
\text { Malmö, Shetland=Inseln, } \\
\text { Westküste Englands, Ka: } \\
\text { nal=Inseln, südlich bis kap } \\
\text { Breton. In der jetzigen } \\
\text { Fauna also eine deutlich } \\
\text { boreale Art. Auch bei } \\
\text { Helgoland (nur leere Scha= } \\
\text { len). Gewöhnlich in Tiefen } \\
\text { von } 12 \text { bis } 180 \mathrm{M} \text {. }\end{array}$ \\
\hline
\end{tabular}

BEMERKUNGEN

Die unterpliocänen For men der Pecten maximus sind die Varietäten Wes: tendorpi Nyst und grandis Sow., die mittelpliocäne Form ist die Varietät complanatus Sow. Es lie= gen junge Schalen und Fragmente dieser letzteren Form aus dem mittleren Pliocän der Gegend Grave= Oss vor.

Diese Art ist ziemlich variabel und bildet meh= rere Varietäten. Wird von Lorié aus I b (Goes) und III a (Utrecht und Amsterdam) erwähnt.

Ist bloss in I b und II a in situ und verschwindet in den jüngeren Stufen. Ein Fragment wird von LORIÉ aus III a (Amster: dam) erwähnt, offenbar auf secundärer Lagerstätte.

Diese Art findet sich nur ziemlich selten in II a der Gegend Grave=Oss, ist im unteren Pliocän Englands und Belgiens häufig. Die ganz glatte Form (var. laevis Penn.) kenne ich bis jetzt aus unsrem Pliocän nicht. 
$\mathrm{S}=$ „selten" bis „sehr selten".

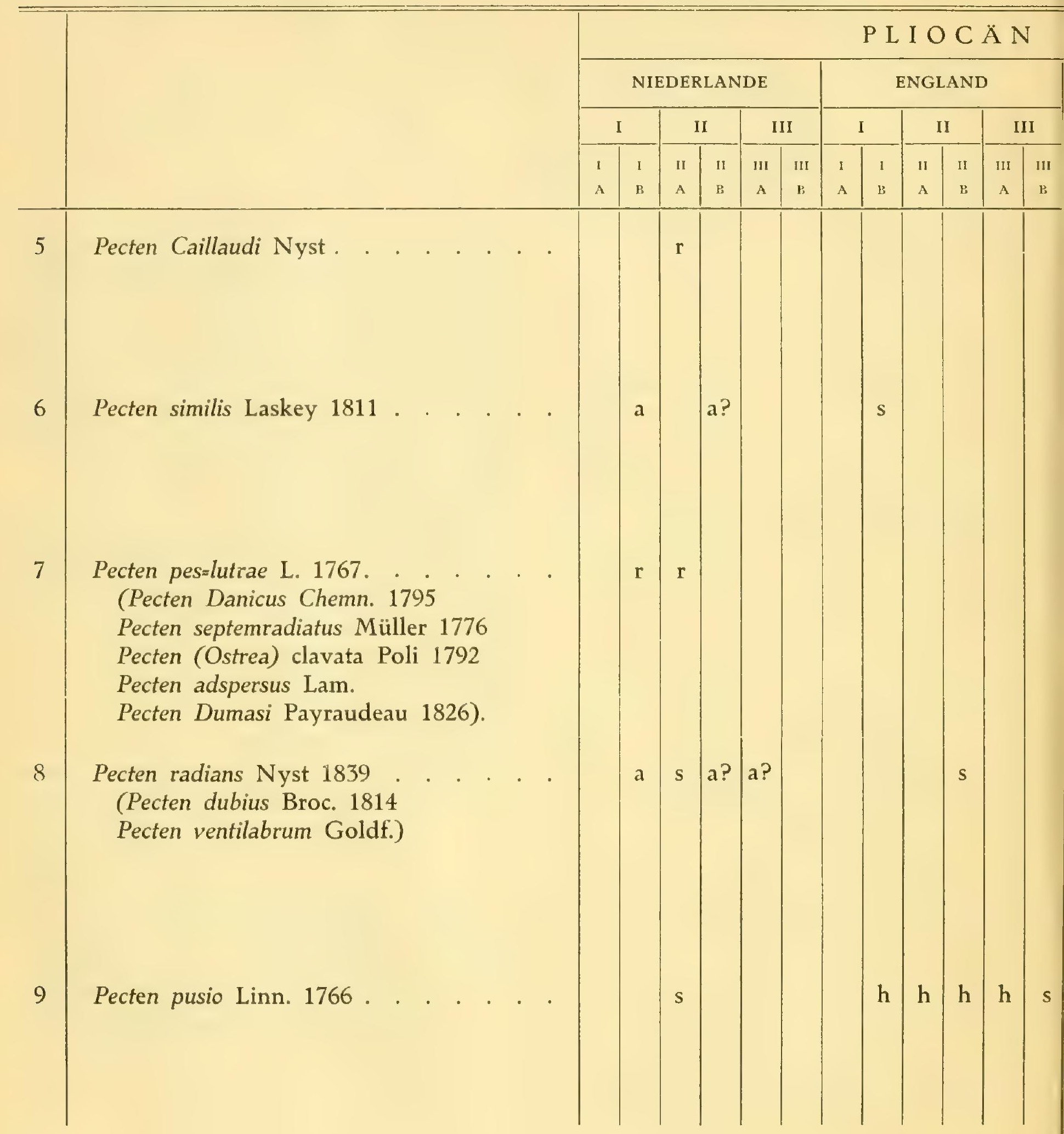


$\mathrm{r}=$ auf secundärer Lagerstätte.

$\mathrm{a}=$ anwesend, jedoch unbekannt ob häufig oder selten.

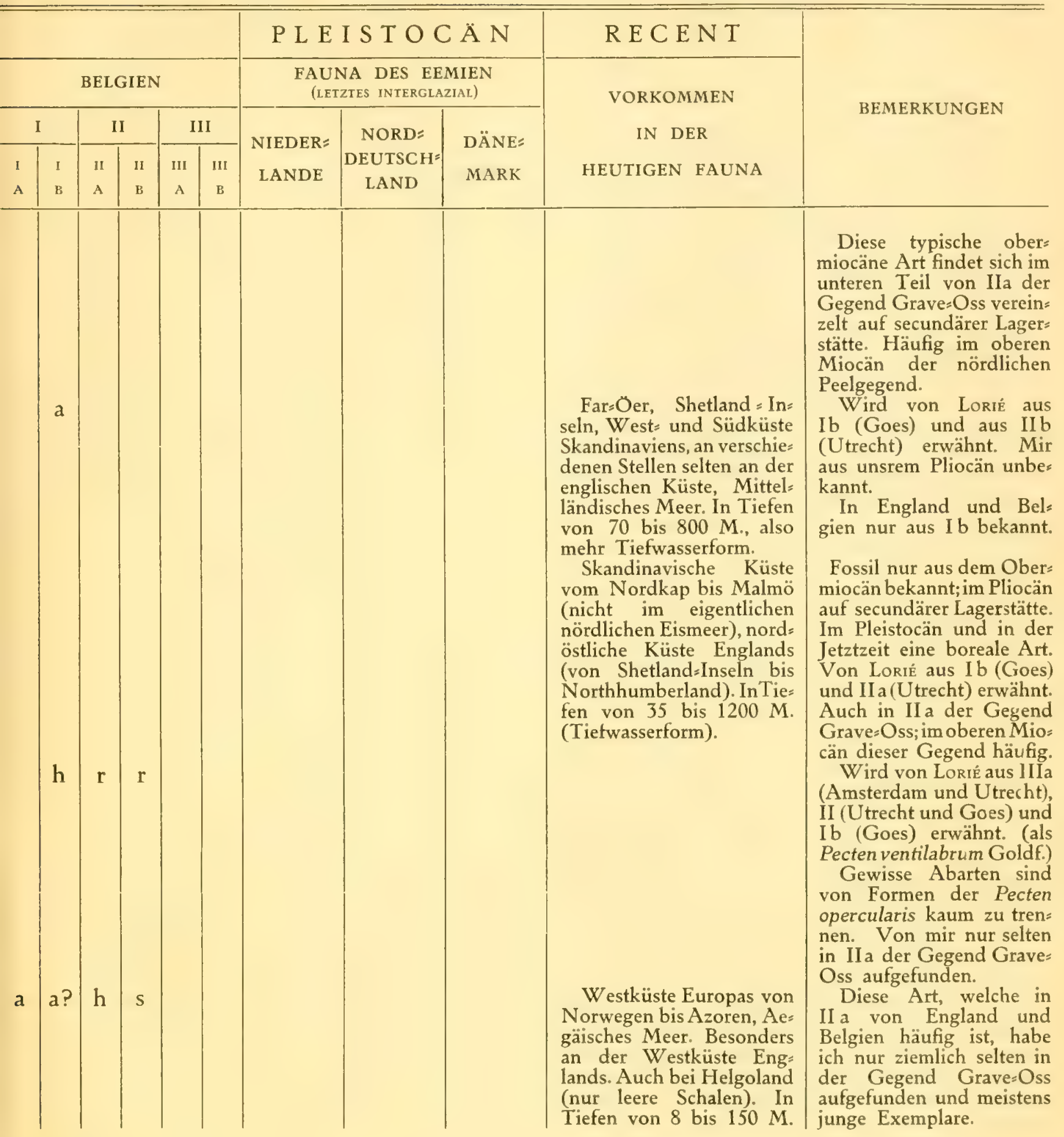


$\mathrm{h}=$,sehr häufig" bis "nicht selten".

$\mathrm{S}=$ „selten" bis „sehr selten".

\begin{tabular}{|c|c|c|c|c|c|c|c|c|c|c|c|c|c|}
\hline & & \multicolumn{12}{|c|}{ P L I O C Ä N } \\
\hline & & \multicolumn{6}{|c|}{ NIEDERLANDE } & \multicolumn{6}{|c|}{ ENGLAND } \\
\hline & & - & & & & II & & & L & & I & & 11 \\
\hline & & $\begin{array}{l}\mathrm{I} \\
\mathrm{A}\end{array}$ & $\begin{array}{l}\text { I } \\
\text { B }\end{array}$ & $\begin{array}{l}\text { II } \\
\text { A }\end{array}$ & $\begin{array}{l}\text { II } \\
\text { B }\end{array}$ & $\begin{array}{l}\text { III } \\
\text { A }\end{array}$ & $\begin{array}{c}\text { III } \\
\text { B }\end{array}$ & I & $\begin{array}{l}\text { I } \\
\text { B }\end{array}$ & $\begin{array}{l}\text { II } \\
\text { A }\end{array}$ & $\begin{array}{l}\text { II } \\
\text { B }\end{array}$ & $\begin{array}{c}111 \\
A\end{array}$ & $\begin{array}{c}\text { III } \\
\text { B }\end{array}$ \\
\hline 10 & Lima Loscombii G. B. Sow. 1842. . . . & & & s & & & & & h & $\mathrm{h}$ & s & s & \\
\hline 11 & 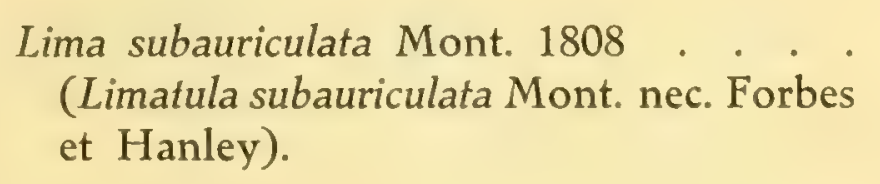 & & a & r? & & & & & $\mathrm{h}$ & & & & \\
\hline 12 & $\begin{array}{l}\text { Pinna rudis Linn. } 1767 \\
\text { (Pinna pectinata bei Wood und Nyst). } \\
\text { Die wahre Pinna pectinata Linn. ist eine } \\
\text { indische Art. }\end{array}$ & & & $\mathrm{h}$ & $\mathrm{h}$ & & & & $\mathrm{h}$ & s & & & \\
\hline 13 & Anomia ephippium Linn. 1767. . . . . & & $\mathrm{h}$ & $\mathrm{h}$ & $\mathrm{h}$ & $\mathrm{h}$ & & & $\mathrm{h}$ & $\mathrm{h}$ & $\mathrm{h}$ & $h=s$ & s \\
\hline 14 & Anomia striata Broc. 1814 . . . . . & & & s & s & & & & h & $\mathrm{h}$ & & $h=s$ & s \\
\hline 15 & Ostrea edulis Linn. I767. . . . . . & & & $\mathrm{h}$ & $\mathrm{s}$ & & & & h & h & & $h=s$ & s \\
\hline
\end{tabular}




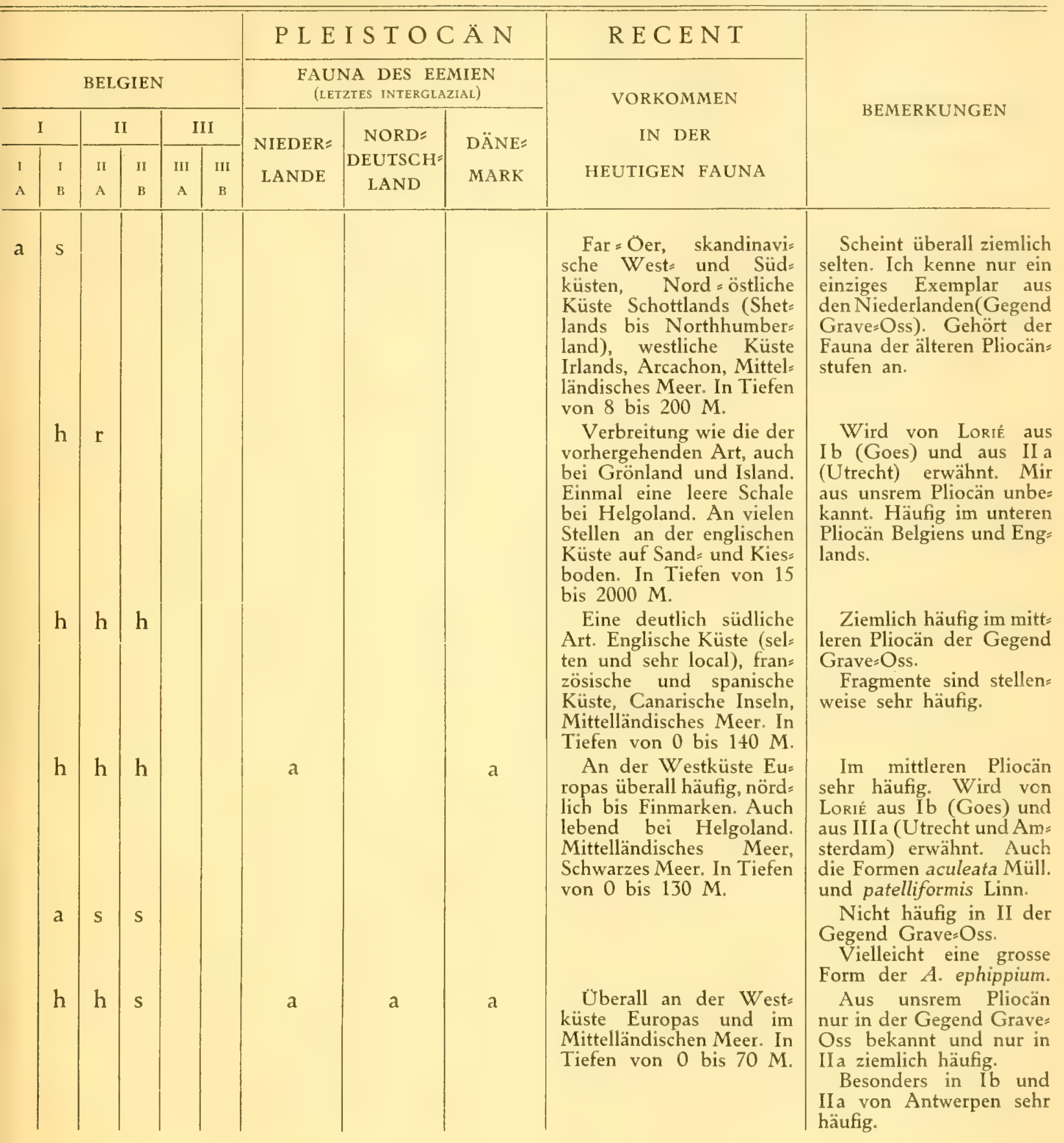


$\mathrm{h}=$ „sehr häufig" bis "nicht selten".

$\mathrm{S}=$,,selten" bis „sehr selten”.

\begin{tabular}{|c|c|c|c|c|c|c|c|c|c|c|c|c|c|c|c|}
\hline & & & & \multicolumn{12}{|c|}{ P L I O C $\ddot{N}$} \\
\hline & & & & \multicolumn{6}{|c|}{ NIEDERLANDE } & \multicolumn{6}{|c|}{ ENGLAND } \\
\hline & & & & & I & & I & & II & & I & & & II & II \\
\hline & & & & $\begin{array}{l}\mathrm{I} \\
\mathrm{A}\end{array}$ & $\begin{array}{l}\mathrm{I} \\
\mathrm{B}\end{array}$ & $\begin{array}{l}11 \\
\text { A }\end{array}$ & $\begin{array}{l}\text { II } \\
\text { B }\end{array}$ & $\begin{array}{r}\text { III } \\
\text { A }\end{array}$ & $\begin{array}{r}\text { III } \\
\text { B }\end{array}$ & $\begin{array}{l}\mathrm{I} \\
\mathrm{A}\end{array}$ & $\begin{array}{l}\text { ' } \\
\text { в }\end{array}$ & $\begin{array}{l}\text { II } \\
\text { A }\end{array}$ & $\begin{array}{l}\text { II } \\
\text { B }\end{array}$ & $\begin{array}{l}\text { III } \\
\text { A }\end{array}$ & \begin{tabular}{|l} 
III \\
B \\
\end{tabular} \\
\hline 16 & Mytilus edulis Linn. 1767 . . . & . & . . & & a? & $\mathrm{h}$ & $\mathrm{h}$ & $\mathrm{h}$ & & & $\mathrm{h}$ & $\mathrm{h}$ & $\mathrm{h}$ & $\mathrm{h}$ & $\mathrm{h}$ \\
\hline 17 & Nucula laevigata Sow. 1818 . . & . & . . & & a & $\mathrm{h}$ & $\mathrm{h}$ & a & & & s & $\mathrm{h}$ & $\mathrm{h}$ & $h=s$ & \\
\hline 18 & Nucula Cobboldiae Sow 1818 . . & . & . . & & & $a ?$ & a? & $\mathrm{h}$ & & & & & & $\mathrm{h}$ & $\mathrm{h}$ \\
\hline 19 & $\begin{array}{l}\text { Nucula nucleus Linn. } 1767 . \\
\text { (Nucula margaritacea Lam. 1801). }\end{array}$ & & . & & a & $\mathrm{h}$ & h & & & & $\mathrm{h}$ & $\mathrm{h}$ & h & $h$ & $s$ \\
\hline 20 & $\begin{array}{l}\text { Nucula proxima Say } 1822 \\
\text { (Nucula trigonula Wood 1840). }\end{array}$ & 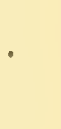 & 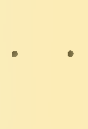 & & & $s$ & & & & & $\mathrm{~h}$ & & & & \\
\hline 21 & $\begin{array}{l}\text { Nucula tenuis Mont. } 1808 \\
\text { (Nucula decipiens Philippi). }\end{array}$ & . & 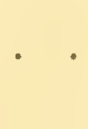 & & & & & $a$ & & & & & & $\mathrm{~s}$ & $\mathrm{~h}$ \\
\hline
\end{tabular}


$r=$ auf secundärer Lagerstätte.

$a=$ anwesend, jedoch unbekannt ob häufig oder selten.

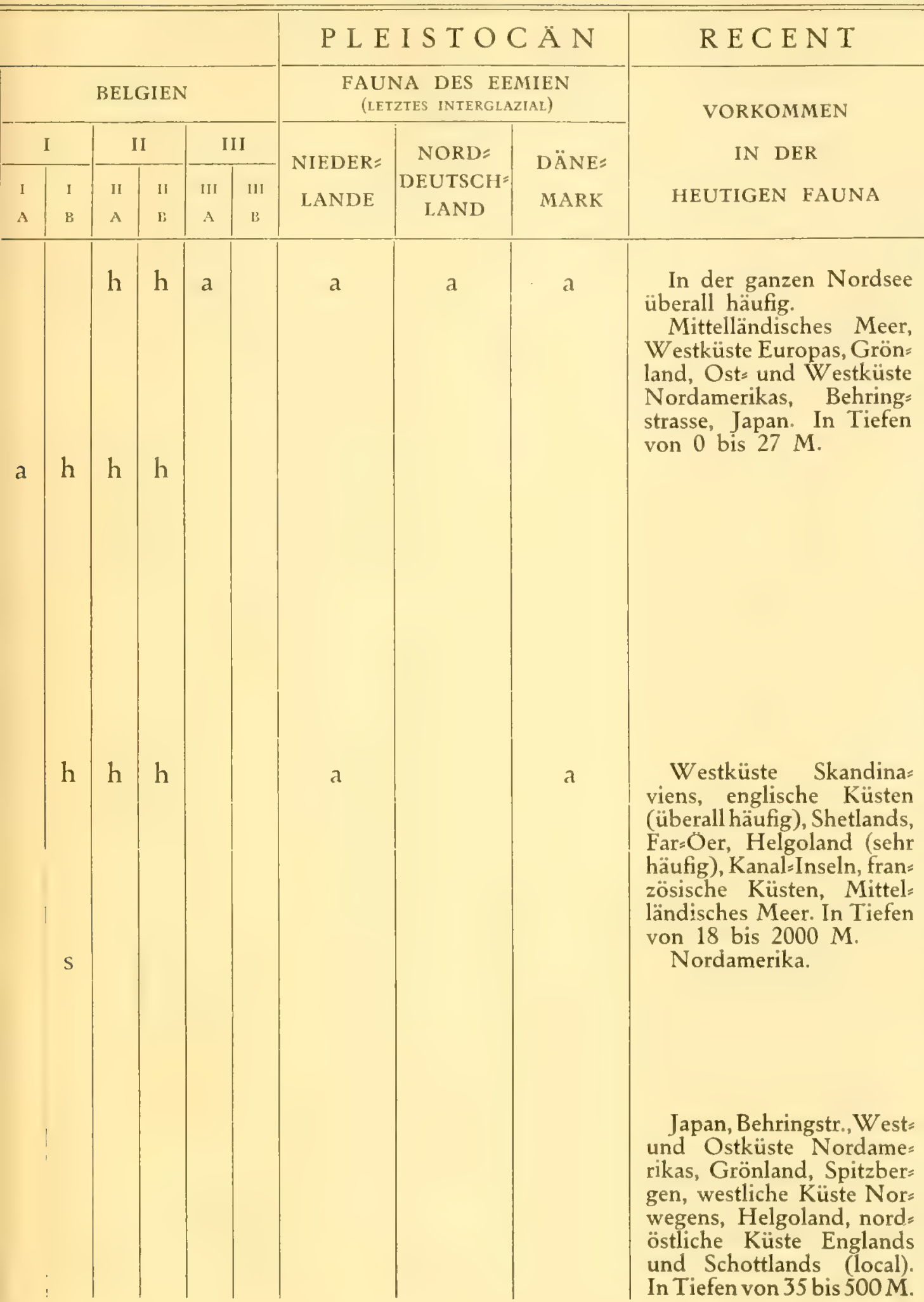

BEMERKUNGEN

Wird von LORIÉ aus III a (Amsterdam, Utrecht, Gorkum) und aus Ib (Goes) erwähnt. An der letzteren Stelle vielleicht aus höheren Schichten stammend.

Wird von LORIÉ aus III a (Amsterdam, Utrecht) und Ib (Goes) erwähnt. Im mittleren Pliocän über= all häufig, wie auch im Miocän.

In unsrem Boden in IIIa überall ziemlich häufig. Wird von LoRIÉ auch aus II a und IIb (Utrecht) er= wähnt, hier wahrscheinlich aus höheren Schichten stammend.

In unsrem Boden im mittleren Pliocän überall häufig. Wird von LORIÉ aus Ib (Goes) erwähnt. Aus dem oberen Pliocän in unsrem Boden bis jetzt unbekannt.

Einzelne Exemplare dieser Art fand ich in IIa der Gegend Grave:Oss.

In England und Belgien nur aus dem unteren Plios cän bekannt. Vielleicht eine Form der Nucula nucleus.

Wird von LORIÉ aus III a (Amsterdam, Utrecht) erwähnt. Mir aus unsrem Pliocän unbekannt. Diese Art gehört der oberen Pliocänfauna an. 
$\mathrm{h}=$ „sehr häufig” bis „nicht selten”.

$\mathrm{S}=$ „selten" bis „sehr selten".

\begin{tabular}{|c|c|c|c|c|c|c|c|c|c|c|c|c|c|c|c|}
\hline & & & & \multicolumn{12}{|c|}{ P L I O C $\ddot{A} N$} \\
\hline & & & & \multicolumn{6}{|c|}{ NIEDERLANDE } & \multicolumn{6}{|c|}{ ENGLAND } \\
\hline & & & & & & & I & & & & 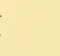 & & I & & II \\
\hline & & & & I & $\begin{array}{l}\mathrm{I} \\
\mathrm{B}\end{array}$ & $\begin{array}{l}\text { II } \\
\text { A }\end{array}$ & $\begin{array}{l}\text { "I } \\
\text { B }\end{array}$ & ${ }_{\text {III }}$ & $\begin{array}{c}{ }^{1 I I} \\
\end{array}$ & $\stackrel{1}{1}$ & $\begin{array}{l}1 \\
\text { в }\end{array}$ & $\begin{array}{l}\text { II } \\
\text { A }\end{array}$ & $\begin{array}{l}\text { II } \\
\text { B }\end{array}$ & ${ }_{\mathrm{III}}$ & $\begin{array}{c}\text { Ii } \\
\text { Bi }\end{array}$ \\
\hline 22 & Leda lanceolata Sow. 1817 . & . & . & & & & & $\mathrm{h}$ & & & & & & $\mathrm{h}$ & $\mathrm{h}$ \\
\hline 23 & $\begin{array}{l}\text { Leda myalis Couth. } 1839 \\
\quad \text { (Leda oblongöides Wood 1840). }\end{array}$ & . & & & & & & a & & & & & & $\mathrm{h}$ & \\
\hline 24 & Leda semistriata Wood 1840 . & . & . & & & $\mathrm{h}$ & $\mathrm{h}$ & a? & & & $\mathrm{h}$ & & & & \\
\hline 25 & $\begin{array}{l}\text { Leda minuta Mont. } 1803 . \\
\text { (Leda caudata Donov. 1802? } \\
\text { Leda pernula Müller 1779?) }\end{array}$ & • & • & & & & & $\mathrm{a}$ & & & & & & s & \\
\hline 26 & Arca Diluvii Lam. . . & & $\cdot$ & & & $\mathrm{r}$ & & & & $\mathrm{h}$ & & & & & \\
\hline
\end{tabular}


$\mathrm{h}=$,sehr häufig" bis ,nicht selten".

$\mathrm{S}=$ "selten" bis "sehr selten".

\begin{tabular}{|c|c|c|c|c|c|c|c|c|c|c|c|c|c|c|c|}
\hline & & & & \multicolumn{12}{|c|}{ P L I O C $\ddot{A} \mathrm{~N}$} \\
\hline & & & & \multicolumn{6}{|c|}{ NIEDERLANDE } & \multicolumn{6}{|c|}{ ENGLAND } \\
\hline & & & & \multicolumn{2}{|c|}{ I } & \multicolumn{2}{|c|}{ II } & \multicolumn{2}{|c|}{ III } & \multicolumn{2}{|c|}{ I } & \multicolumn{2}{|c|}{ II } & \multicolumn{2}{|c|}{ III } \\
\hline & & & & 1 & $\begin{array}{l}\text { I } \\
\text { B }\end{array}$ & $\begin{array}{l}\text { II } \\
\text { A }\end{array}$ & $\begin{array}{l}\text { II } \\
\text { B }\end{array}$ & $\begin{array}{c}\mathrm{III} \\
\mathrm{A}\end{array}$ & $\begin{array}{c}\text { III } \\
\text { B }\end{array}$ & $\begin{array}{l}\text { I } \\
\text { A }\end{array}$ & $\begin{array}{l}\mathrm{I} \\
\mathrm{B}\end{array}$ & $\begin{array}{l}\text { II } \\
\text { A }\end{array}$ & $\begin{array}{l}\text { II } \\
\text { B }\end{array}$ & III & $\begin{array}{c}\text { III } \\
\text { B }\end{array}$ \\
\hline 27 & $\begin{array}{l}\text { Cucullaea pectunculöides Scac. } 1834 \text {. } \\
\text { (Arca pectunculöides Scac.) }\end{array}$ & $\cdot$ & & & & $\mathrm{r}$ & & & & & h & & & & \\
\hline 28 & $\begin{array}{l}\text { Pectunculus glycimeris Linn. } 1767 . \\
\quad \text { (Pectunculus variabilis J. Sow. 1825) }\end{array}$ & . & . & & & $\mathrm{h}$ & $\mathrm{h}$ & $\mathrm{a}$ & & $\mathrm{h}$ & $\mathrm{h}$ & h & $\dot{\mathrm{h}}$ & h & s \\
\hline 29 & Pectunculus pilosus Linn. 1767. . . & . & . & & a & r & & & & & $\mathrm{h}$ & $\mathrm{h}$ & $\mathrm{h}$ & $h=s$ & \\
\hline 30 & $\begin{array}{l}\text { Limopsis aurita Broc. } 1814 . \\
\quad \text { (Limopsis sublaevigata Nyst I839). }\end{array}$ & . & . & & & $\mathbf{r}$ & & & & & $\mathrm{h}$ & & r & $\mathbf{r}$ & $\mathrm{r}$ \\
\hline 31 & $\begin{array}{l}\text { Limopsis anomala Eichw. } 1830 \\
\quad \text { (Limopsis pygmaea Phil. 1836). }\end{array}$ & 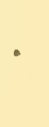 & - & & $\mathbf{a}$ & $\mathbf{r}$ & & & & & $\mathrm{h}$ & $\mathrm{r}$ & $\mathrm{r}$ & $\mathbf{r}$ & \\
\hline 32 & Cardita senilis Lam. . . . . . . & $\cdot$ & $\cdot$ & & a & & & & & $\mathrm{h}$ & $\mathrm{h}$ & $\begin{array}{l}\mathrm{h} \\
\mathrm{r} ?\end{array}$ & $\begin{array}{c}\mathrm{h} \\
\mathrm{r} ?\end{array}$ & $\begin{array}{c}\mathrm{h} \\
\mathrm{r} ?\end{array}$ & $\mathrm{r}$ \\
\hline
\end{tabular}




\begin{tabular}{|c|c|c|c|c|c|c|c|c|c|c|}
\hline & & & & & & \multicolumn{3}{|c|}{ P L E I S T O C A N } & RECENT & \multirow{4}{*}{ BEMERKUNGEN } \\
\hline \multicolumn{6}{|c|}{ BELGIEN } & \multicolumn{3}{|c|}{$\begin{array}{l}\text { FAUNA DES EEMIEN } \\
\text { (IETZTES INTERGLAZIAL) }\end{array}$} & \multirow{2}{*}{$\begin{array}{l}\text { VORKOMMEN } \\
\text { IN DER }\end{array}$} & \\
\hline \multicolumn{2}{|c|}{ I } & \multicolumn{2}{|c|}{ II } & \multicolumn{2}{|c|}{ III } & \multirow{2}{*}{$\begin{array}{l}\text { NIEDER= } \\
\text { LANDE }\end{array}$} & \multirow{2}{*}{$\begin{array}{c}\text { NORD }= \\
\text { DEUTSCH }= \\
\text { LAND }\end{array}$} & \multirow{2}{*}{$\begin{array}{l}\text { DÄNE= } \\
\text { MARK }\end{array}$} & & \\
\hline $\begin{array}{l}\mathrm{I} \\
\mathrm{A}\end{array}$ & $\begin{array}{l}1 \\
\mathrm{e}\end{array}$ & "I & $\begin{array}{l}\text { is } \\
\text { B }\end{array}$ & $\begin{array}{c}\text { III } \\
\mathrm{A}\end{array}$ & $\begin{array}{c}\text { III } \\
\mathrm{B}\end{array}$ & & & & HEUTIGEN FAUNA & \\
\hline & $\mathrm{h}$ & & & & & & & & $\begin{array}{l}\text { In der jetzigen Fauna } \\
\text { eine vorwiegend boreale } \\
\text { Form. Grönland, Finmar } \\
\text { ken, Shetlands, Hebriden, } \\
\text { Westküste Irlands. Auch } \\
\text { Gibraltar und Aegäisches } \\
\text { Meer. In Tiefen von } 35 \text { bis } \\
350 \mathrm{M} \text {. }\end{array}$ & $\begin{array}{l}\text { In II a der Gegend } \\
\text { Grave=Oss selten auf se= } \\
\text { cundärer Lagerstätte (aus } \\
\text { dem Miocän stammend). } \\
\text { Im unteren Pliocän Bel= } \\
\text { giens und Englands häufig. }\end{array}$ \\
\hline & & $\mathrm{h}$ & h & $a$ & & & & & $\begin{array}{l}\text { Westliche Küste Skandi= } \\
\text { naviens und Grossbritan= } \\
\text { niens, östliche Küste Gross= } \\
\text { britannien (ziemlich selten) } \\
\text { Madeira und Canarische } \\
\text { Inseln, Mittelländ. Meer. } \\
\text { In Tiefen von } 10 \text { bis } 150 \mathrm{M} \text {. }\end{array}$ & $\begin{array}{l}\text { Uberall im mittleren } \\
\text { Pliocän häufig. In der } \\
\text { Gegend von Breda von } \\
\text { mir auch in III a gefunden. }\end{array}$ \\
\hline a & $\mathrm{h}$ & $\mathrm{r}$ & $\mathrm{r}$ & & & & & & $\begin{array}{l}\text { Wahrscheinlich eine an: } \\
\text { geschwollene Form der } P \text {. } \\
\text { glycimeris. }\end{array}$ & $\begin{array}{l}\text { Im mittleren und oberen } \\
\text { Pliocän offenbar auf se } \\
\text { cundärer Lagerstätte. Von } \\
\text { LoRIé aus I b (Goes) } \\
\text { erwähnt. Wahrscheinlich } \\
\text { nichts anderes als eine Va= } \\
\text { rictät der vorhergehenden } \\
\text { Art. (Tiefwasserform?) }\end{array}$ \\
\hline a & s & & & & & & & & $\begin{array}{l}\text { Shetland=Inseln, subfos: } \\
\text { sil an der westlichen Küste } \\
\text { Irlands in einer Tiefe von } \\
550 \text { M. Mittelländisches } \\
\text { Meer. }\end{array}$ & $\begin{array}{l}\text { In II und III auf se= } \\
\text { cundärer Lagerstätte. Häus } \\
\text { fig an einer Stelle im uns } \\
\text { teren Pliocän Englands und } \\
\text { überall im Miocän. }\end{array}$ \\
\hline & $\mathrm{h}$ & $r$ & & & & & & & $\begin{array}{l}\text { Mittelländisches Meer. } \\
\text { Mittelländisches Meer. }\end{array}$ & $\begin{array}{l}\text { Wird von LoRIÉ aus Ib } \\
\text { (Goes) erwähnt. In II und } \\
\text { III auf secundärer Lager } \\
\text { stätte. Häufig an einer } \\
\text { Stelle in Ib Englands und } \\
\text { im Miocän. } \\
\text { Wird von LoRIÉ aus Ib } \\
\text { (Goes) erwähnt; mir aus } \\
\text { unsrem Pliocän unbekannt. } \\
\text { In II und III Englands } \\
\text { sehr wahrscheinlich auf } \\
\text { secundärer Lagerstätte. }\end{array}$ \\
\hline
\end{tabular}


$\mathrm{h}=$ „sehr häufig” bis ,, nicht selten”.

$\mathbf{s}=$ „selten” bis „sehr selten”.

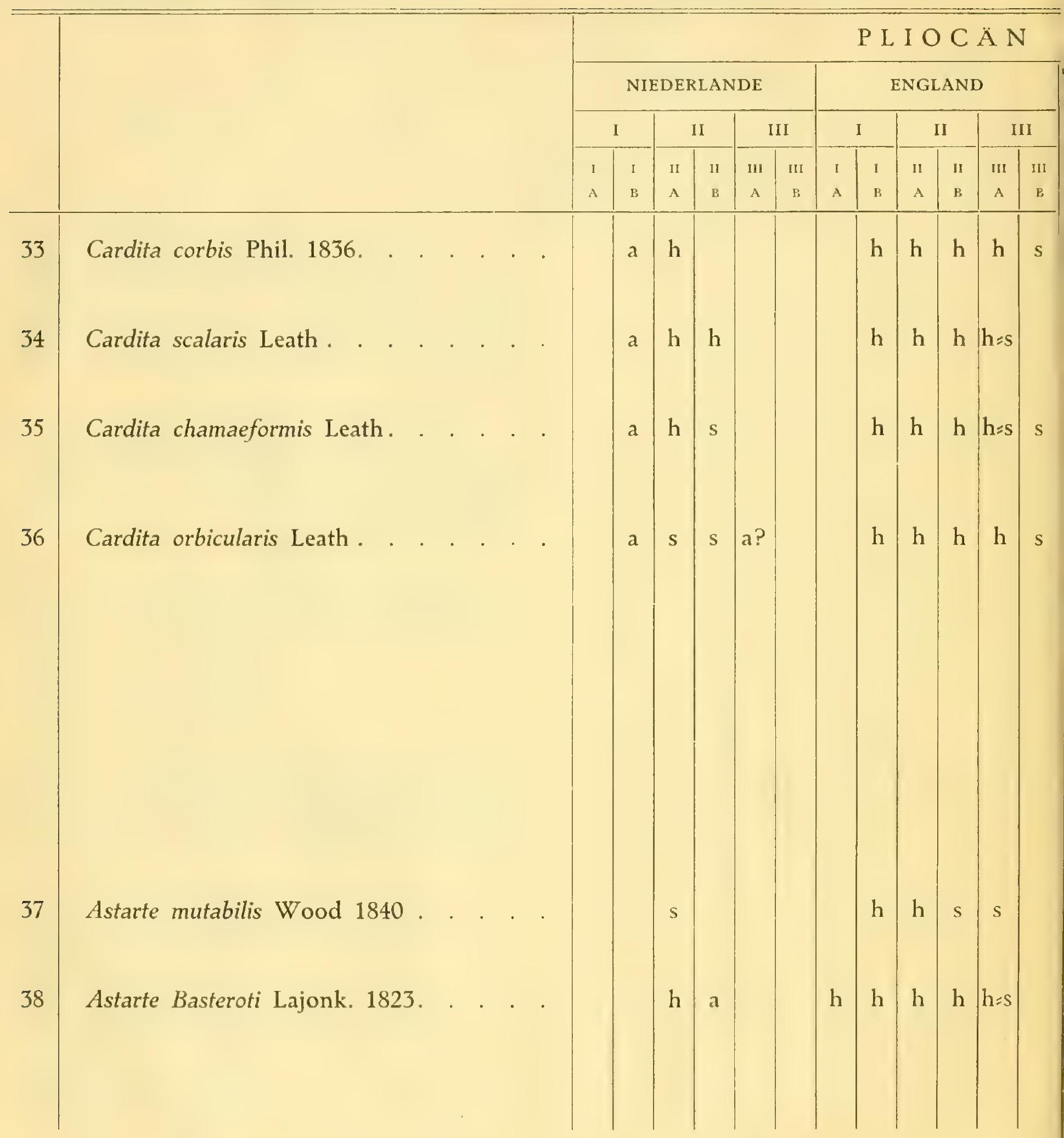


$\mathrm{r}=$ auf secundärer Lagerstätte.

$a=$ anwesend, jedoch unbekannt ob häufig oder selten.

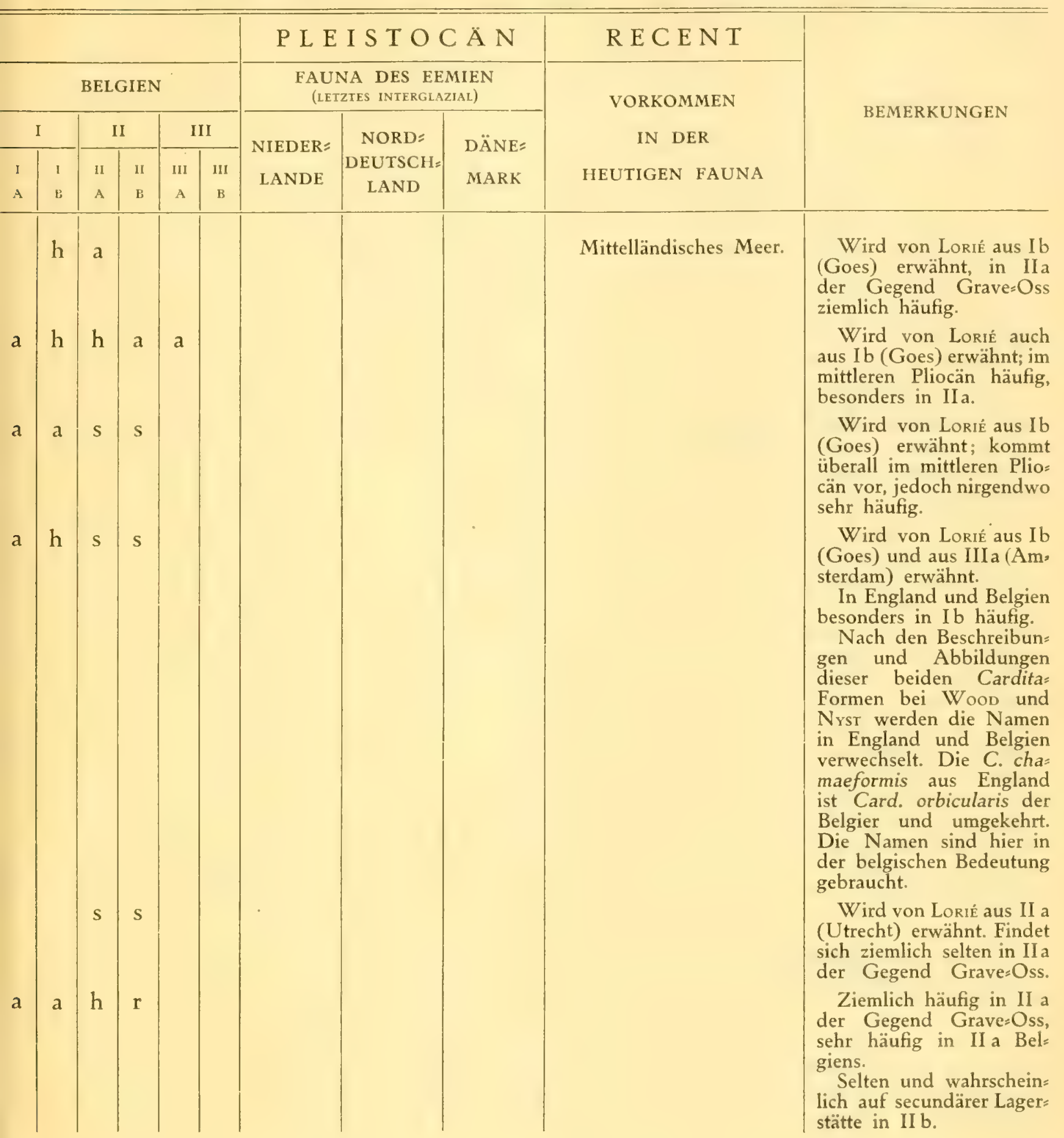


h = „sehr häufig” bis ,nicht selten".

$\mathrm{S}=$ "selten" bis "sehr selten".

\begin{tabular}{|c|c|c|c|c|c|c|c|c|c|c|c|c|c|c|c|c|c|}
\hline & & & & & & \multicolumn{12}{|c|}{ P L I OC $\ddot{A} N$} \\
\hline & & & & & & \multicolumn{6}{|c|}{ NIEDERLANDE } & \multicolumn{6}{|c|}{ ENGLAND } \\
\hline & & & & & & \multicolumn{2}{|c|}{ I } & \multicolumn{2}{|c|}{ II } & \multicolumn{2}{|c|}{ III } & \multicolumn{2}{|c|}{ I } & \multicolumn{2}{|c|}{ II } & \multicolumn{2}{|c|}{ III } \\
\hline & & & & & & I & $\begin{array}{l}\text { I } \\
\text { b3 }\end{array}$ & $\begin{array}{l}\text { II } \\
\text { A }\end{array}$ & $\begin{array}{l}\mathrm{II} \\
\mathrm{B}\end{array}$ & $\begin{array}{l}\text { III } \\
\text { A }\end{array}$ & $\begin{array}{c}\text { III } \\
\text { B }\end{array}$ & I & $\begin{array}{l}\mathrm{I} \\
\mathrm{B}\end{array}$ & $\begin{array}{l}\text { II } \\
\text { A }\end{array}$ & $\begin{array}{l}\text { II } \\
\text { B }\end{array}$ & A & \\
\hline 39 & Astarte Omaliusi Lajonk. 1823. & - & • & . & - & & a & $\mathrm{h}$ & s & & & $\mathrm{h}$ & $\mathrm{h}$ & h & $\mathrm{h}$ & $\mathrm{h}$ & \\
\hline 40 & $\begin{array}{l}\text { Astarte Galeotti Nyst } 1835 \\
\quad \text { (Astarte gracilis Münst.) }\end{array}$ & • & . & . & . & & a & $\mathrm{h}$ & $\mathrm{h}$ & $\mathrm{a}$ & & $\mathrm{h}$ & h & h & $\mathrm{h}$ & $\mathrm{h}$ & \\
\hline 41 & Astarte Burtini Lajonk. 1823 . & - & - & . & . & & & s & & & & & $\mathrm{h}$ & h & $\mathrm{h}$ & $\mathrm{h}$ & \\
\hline 42 & Astarte obliquata Sow. 1817 . & . & ${ }^{\circ}$ & . & . & & & $\mathrm{h}$ & & & & & & $?$ & & & \\
\hline 43 & Astarte incerta Wood 1848. & • & - & . & . & & & h & $\mathrm{h}$ & & & & $\mathrm{h}$ & $\mathrm{h}$ & h & s & \\
\hline 44 & Astarte corbulöides Lajonk. 1823 & & • & . & . & & a & a? & a? & & & & & & $\mathrm{r}$ & & \\
\hline
\end{tabular}


h = „sehr häufig" bis ,nicht selten".

$s=$ „selten" bis „sehr selten".

\begin{tabular}{|c|c|c|c|c|c|c|c|c|c|c|c|c|c|c|c|c|}
\hline & & & & & & \multicolumn{11}{|c|}{ P L I OC $\ddot{A} \mathrm{~N}$} \\
\hline & & & & & & \multicolumn{6}{|c|}{ NIEDERLANDE } & \multicolumn{5}{|c|}{ ENGLAND } \\
\hline & & & & & & \multicolumn{2}{|c|}{ I } & \multicolumn{2}{|c|}{ II } & \multicolumn{2}{|c|}{ III } & \multicolumn{2}{|c|}{ I } & \multicolumn{2}{|c|}{ II } & III \\
\hline & & & & & & $\begin{array}{l}\mathrm{I} \\
\mathrm{A}\end{array}$ & $\begin{array}{l}\mathrm{I} \\
\mathrm{B}\end{array}$ & $\begin{array}{l}\text { II } \\
\mathrm{A}\end{array}$ & $\begin{array}{l}\text { II } \\
\text { E }\end{array}$ & ${ }_{\mathrm{A}}^{\mathrm{M}}$ & $\begin{array}{c}\text { III } \\
\text { B }\end{array}$ & $\begin{array}{l}\mathrm{I} \\
\mathrm{A}\end{array}$ & $\begin{array}{l}\text { I } \\
\text { B }\end{array}$ & II & $\begin{array}{l}\text { II } \\
\text { B }\end{array}$ & $\begin{array}{l}\text { III } \\
\text { A }\end{array}$ \\
\hline 45 & Astarte trigonata Nyst. . . . & . & . & . & . & & & $\mathrm{h}$ & & & & & & & $s$ & \\
\hline 46 & Astarte incrassata Broc. 1814 (?) & . & . & · & . & & & $\mathrm{r}$ & & & & & $\mathrm{h}$ & $s$ & s & \\
\hline 47 & $\begin{array}{l}\text { Astarte pygmaea Münst } \\
\quad \text { (Astarte Waeli Nyst). }\end{array}$ & 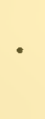 & . & - & . & & a & $a$ & & & & & $\mathrm{~h}$ & s & & \\
\hline 48 & $\begin{array}{l}\text { Astarte triangularis Mont. } 1803 \\
\text { (Astarte laevigata von Münst? }\end{array}$ & & . & • & . & & a & $\mathrm{s}$ & & & & & $\mathrm{h}$ & $\mathrm{h}$ & $\mathrm{s}$ & $s$ \\
\hline 49 & Woodia excurrens Wood 1848. & . & $\cdot$ & $\cdot$ & . & & $a$ & $a$ & & & & & $\mathbf{s}$ & s & & \\
\hline 50 & Woodia digitaria Linn. 1767 & . & $\cdot$ & . & . & & $a$ & $\mathrm{~h}$ & $\mathrm{~h}$ & $a$ & & & $\mathrm{~h}$ & $\mathrm{~h}$ & $\mathrm{~h}$ & h \\
\hline
\end{tabular}


$\mathrm{r}=$ auf secundärer Lagerstätte.

$\mathrm{a}=$ anwesend, jedoch unbekannt ob häufig oder selten.

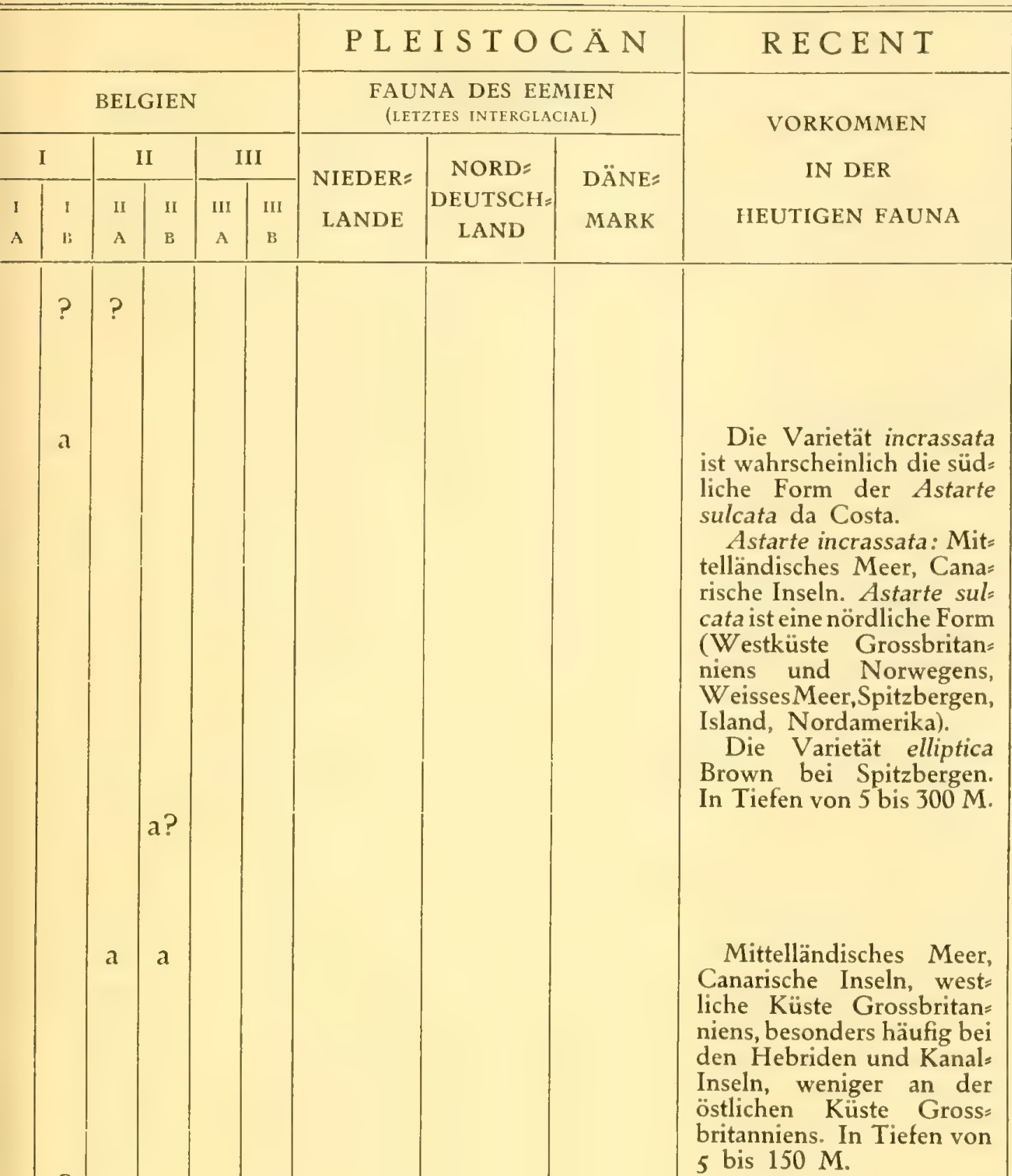
5 bis $150 \mathrm{M}$.

Südküste Englands (nur leere Schalen), Mittellän= disches Meer.
Diese typische Art fand ich nicht selten in II a der Gegend Grave=Oss; scheint in England und Belgien sehr selten.

Exemplare einer $A$ starte: Art, welche im Obermio= cän sehr häufig ist und der Astarte incrassata Broc. sehr nahe steht, fin= den sich gelegentlich in II a auf secundärer Lager= stätte.

Wird von Lorié aus Ib (Goes) erwähnt; auch aus II a der Gegend von Grave bekannt. Besonders häufig im Obermiocän.

Wird von Lorié aus Ib (Goes) und aus II a (Goes und Grave) erwähnt. Auch von mir in II a der Gegend Grave $=$ Oss aufgefunden.

Wird von LORIÉ aus Ib (Goes) und II a (Grave) erwähnt.

Mir aus unsrem Pliocän unbekannt.

Wird von Lorié aus Ib (Goes) und III a (Gorkum) erwähnt.

Im mittleren Pliocän überall sehr häufig. 
$\mathrm{h}=$ „sehr häufig” bis ,nicht selten”.

$\mathrm{s}=$ „selten" bis „sehr selten".

\begin{tabular}{|c|c|c|c|c|c|c|c|c|c|c|c|c|c|c|c|c|}
\hline & & & & & \multicolumn{12}{|c|}{ PL I OC $\ddot{A} \mathrm{~N}$} \\
\hline & & & & & \multicolumn{6}{|c|}{ NIEDERLANDE } & \multicolumn{6}{|c|}{ ENGLAND } \\
\hline & & & & & \multicolumn{2}{|c|}{ I } & \multicolumn{2}{|c|}{ II } & \multicolumn{2}{|c|}{ III } & \multicolumn{2}{|c|}{ I } & \multicolumn{2}{|c|}{ II } & \multicolumn{2}{|c|}{ III } \\
\hline & & & & & $\begin{array}{l}1 \\
\mathrm{~A}\end{array}$ & $\begin{array}{l}\text { I } \\
\text { B }\end{array}$ & $\begin{array}{l}\text { "I } \\
\text { A }\end{array}$ & $\begin{array}{l}\text { II } \\
\text { B }\end{array}$ & $\begin{array}{l}\mathrm{III} \\
\mathrm{A}\end{array}$ & $\begin{array}{c}\text { III } \\
\text { D }\end{array}$ & $\begin{array}{l}\mathrm{I} \\
\mathrm{A}\end{array}$ & $\begin{array}{l}\text { I } \\
\text { B }\end{array}$ & $\begin{array}{l}\text { II } \\
\text { A }\end{array}$ & $\begin{array}{l}\text { II } \\
\text { B }\end{array}$ & $\begin{array}{c}\text { III } \\
\mathrm{A}\end{array}$ & $\begin{array}{l}\text { III } \\
\text { B }\end{array}$ \\
\hline 51 & $\begin{array}{l}\text { Scintilla ambigua Nyst } 1839 . \\
\quad \text { (Kellia ambigua Nyst). }\end{array}$ & . & 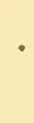 & • & & & h & & & & & $\mathrm{h}$ & h & s & & \\
\hline 52 & $\begin{array}{l}\text { Montacuta ferruginosa Mont. } 1808 \\
\text { (Montacuta ovata Wood 1840). }\end{array}$ & . & . & . & & & $a$ & & & & & h & s & & & \\
\hline 53 & Diplodonta astartea Nyst 1835. & . & . & . & & & s & $\mathrm{h}$ & & & & h & h & s & $\mathrm{h}$ & s \\
\hline 54 & $\begin{array}{l}\text { Diplodonta rotundata Mont. } 1803 . \\
\text { (Diplodonta dilatata Philippi). }\end{array}$ & . & . & . & & & $\mathrm{s}$ & $\mathrm{s}$ & & & & h & s & . & $s$ & \\
\hline 55 & $\begin{array}{l}\text { Diplodonta dilatata Wood } 1840 \\
\text { (Diplodonta Woodi Nyst). }\end{array}$ & . & . & . & & & $\mathrm{h}$ & & & & h & h & s & & s & \\
\hline 56 & Lucina borealis Linn. 1767 . . & . & . & , & & & $\mathrm{h}$ & $\mathrm{h}$ & a & & & $\mathrm{h}$ & h & $\mathrm{h}$ & $\mathrm{h}$ & $h=:$ \\
\hline 57 & Lucina decorata Wood 1848 . & . & - & . & & & s & & & & & $\mathrm{h}$ & s & s & s & \\
\hline 58 & Lucina crenulata Wood 1840 . & . & . & 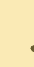 & & & s & & & & & $\mathrm{h}$ & & & & \\
\hline 59 & $\begin{array}{l}\text { Lucina divaricata Linn. } 1767 \text {. } \\
\text { (Loripes arcuata Mont. 1803). }\end{array}$ & & . & 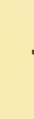 & & & & & h & & & & & & s & h \\
\hline
\end{tabular}




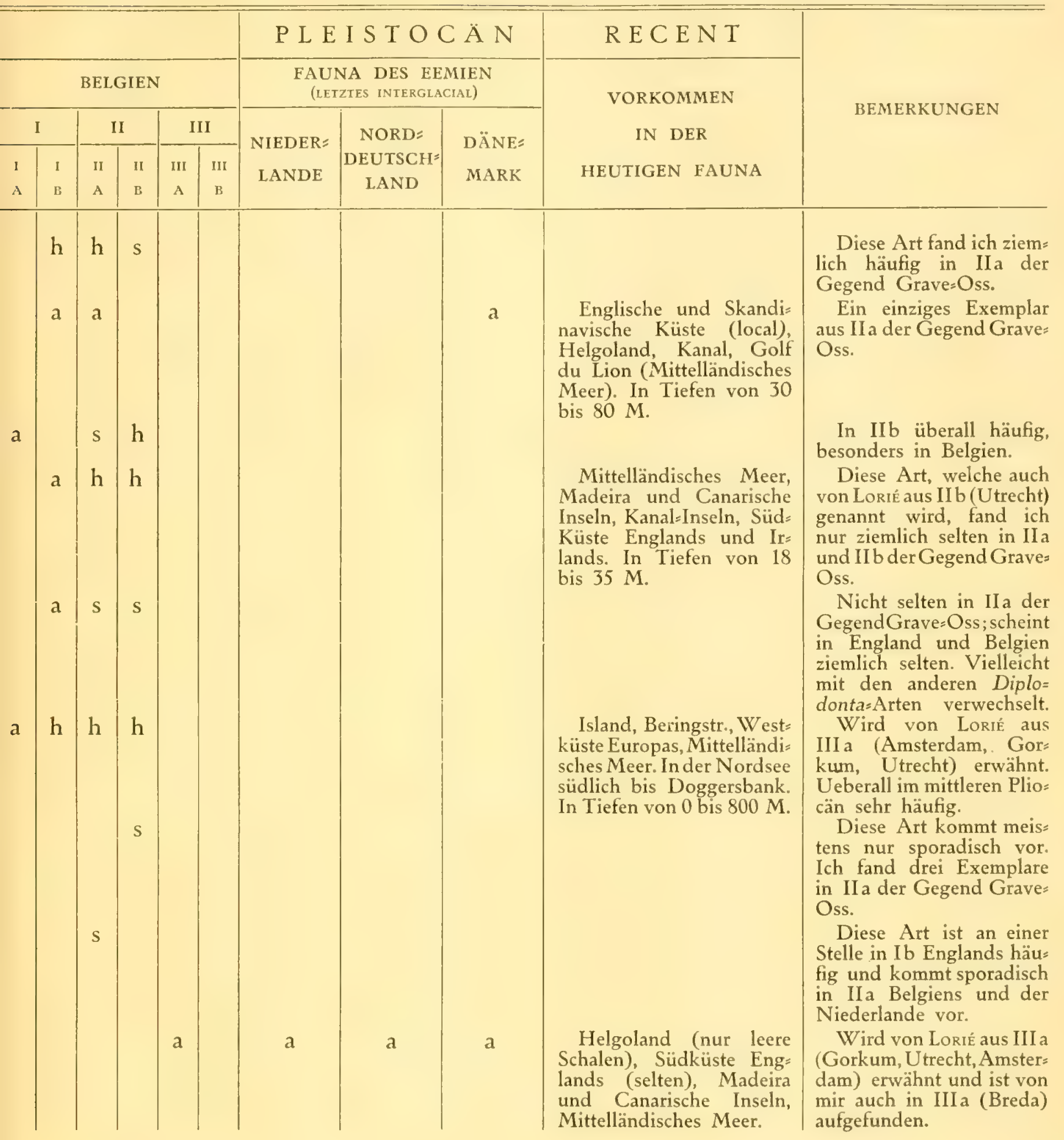


h = „sehr häufig” bis ,nicht selten”.

$\mathbf{S}=$ "selten" bis "sehr selten".

\begin{tabular}{|c|c|c|c|c|c|c|c|c|c|c|c|c|c|c|c|c|}
\hline & & & & & \multicolumn{12}{|c|}{ P L I OC $\AA$ N } \\
\hline & & & & & \multicolumn{6}{|c|}{ NIEDERLANDE } & \multicolumn{6}{|c|}{ ENGLAND } \\
\hline & & & & & \multicolumn{2}{|c|}{ I } & \multicolumn{2}{|c|}{ II } & \multicolumn{2}{|c|}{ III } & \multicolumn{2}{|c|}{ I } & \multicolumn{2}{|c|}{ II } & \multicolumn{2}{|c|}{ III } \\
\hline & & & & & $\begin{array}{l}\mathrm{I} \\
\mathrm{A}\end{array}$ & $\begin{array}{l}\text { I } \\
\text { B }\end{array}$ & $\begin{array}{l}\text { II } \\
\text { A }\end{array}$ & $\begin{array}{l}\text { II } \\
\text { B }\end{array}$ & $\begin{array}{l}\text { III } \\
\text { A }\end{array}$ & $\begin{array}{c}\text { III } \\
\text { B }\end{array}$ & $\begin{array}{l}\mathrm{I} \\
\mathrm{A}\end{array}$ & $\begin{array}{l}\text { I } \\
\text { в }\end{array}$ & $\begin{array}{l}\text { II } \\
\text { A }\end{array}$ & $\begin{array}{l}11 \\
\text { B }\end{array}$ & $\begin{array}{l}\text { III } \\
\text { A }\end{array}$ & $\begin{array}{l}\text { III } \\
\text { B }\end{array}$ \\
\hline 60 & Cardium Parkinsoni Sow. 1814. & . & . & . & & & & h & & & & & $\mathrm{h}$ & $\mathrm{h}$ & h & \\
\hline 61 & Cardium decorticatum S. Wood & 1840 & . & . & & & $\mathrm{h}$ & $\mathrm{h}$ & a & & & h & h & $\mathrm{h}$ & $\mathrm{h}$ & \\
\hline 62 & Cardium Groenlandicum Chemn. & 178 & • & . & & & & & a & & & & & & $s=h$ & $\mathrm{~h}$ \\
\hline 63 & Cardium echinatum Linn. 1767. & . & - & . & & & & & a & & & & s & & s & s \\
\hline 64 & Cardium edule Linn. 1767 . . & . & • & . & & & h & h & $\mathrm{h}$ & & & h & h & h & $\mathrm{h}$ & h \\
\hline 65 & Cardium fasciatum Mont. . . & . . & • & . & & a & $\mathrm{s}$ & s & $\mathrm{a}$ & & & h & h & $\mathrm{s}$ & $s$ & $S$ \\
\hline
\end{tabular}


$\mathrm{r}=$ auf secundärer Lagerstätte.

$a=$ anwesend, jedoch unbekannt ob häufig oder selten.

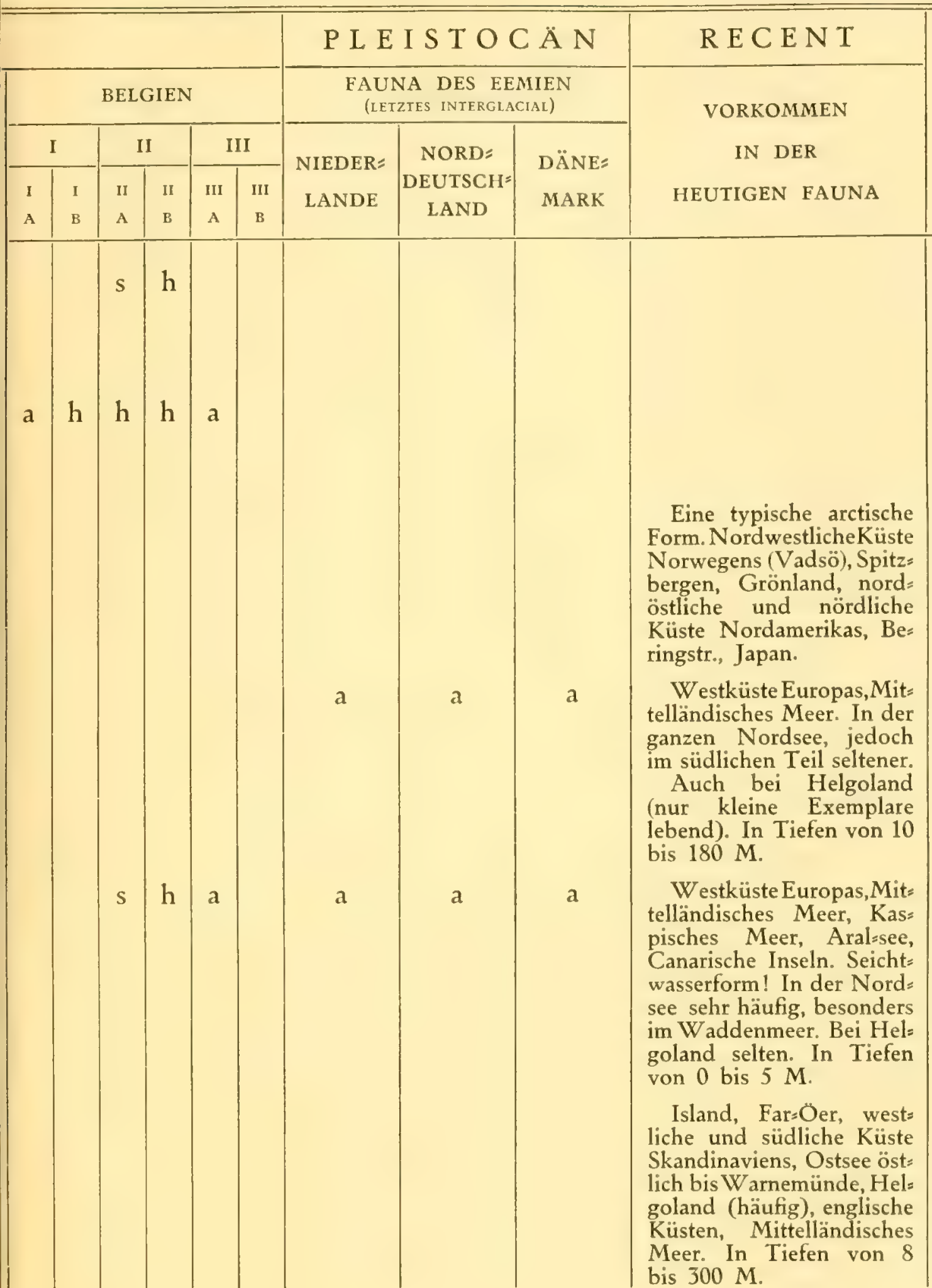

BEMERKUNGEN

Ziemlich häufig in IIb der Gegend von Grave= Oss, jedoch meistens schlecht erhalten und sehr zerbrechlich.

Im mittleren Pliocän überall häufig.

Wird von LORIÉ auch aus III a (Utrecht) erwähnt.

Wird von LORIÉ aus III a (Goes und Utrecht) erwähnt. Mir unbekannt. Nur im oberen Pliocän.

In III a (Oudewater) fand ich 2 Exemplare die= ser Art, welche im oberen Pliocän Englands selten ist.

Wird in IIb und IIIa überall sehr häufig ; in II a spärlicher.

Auch in den diluvialen und alluvialen marinen Ablagerungen in unsrem Boden sehr häufig.

Wird von LORIÉ aus Ib (Goes), IIb (Utrecht) und IIIa (Utrecht) erwähnt. Von mir in II a (Grave) aufgefunden. Ist von Cat= dium nodosum oft schwie= rig zu trennen. 
$\mathrm{h}=$ „sehr häufig” bis ,nicht selten”.

$\mathrm{s}=$,selten" bis „sehr selten”.

\begin{tabular}{|c|c|c|c|c|c|c|c|c|c|c|c|c|c|c|c|c|c|}
\hline & & & & & & & \multicolumn{11}{|c|}{ P L I OCA N } \\
\hline & & & & & & & \multicolumn{6}{|c|}{ NIEDERLANDE } & \multicolumn{5}{|c|}{ ENGLAND } \\
\hline & & & & & & & \multicolumn{2}{|c|}{ I } & \multicolumn{2}{|c|}{ II } & \multicolumn{2}{|c|}{ III } & \multicolumn{2}{|c|}{ I } & \multicolumn{2}{|c|}{ II } & III \\
\hline & & & & & & & A & $\begin{array}{l}\mathrm{I} \\
\mathrm{B}\end{array}$ & A & $\begin{array}{l}\text { II } \\
\text { B }\end{array}$ & III & B & I & $\begin{array}{l}\text { I } \\
\text { B }\end{array}$ & II & II & II \\
\hline 66 & Cardium nodosum Turt. 1822 & . & . & . & - & . & & & $\mathbf{s}$ & & a? & & & $\mathrm{h}$ & $\mathrm{h}$ & s & s \\
\hline 67 & Cardium subturgidum d'Orb. & . & . & . & • & . & & & $\mathbf{r}$ & & a? & & & & & & \\
\hline 68 & Isocardia cor Linn. 1767 . . & . & . & . & • & . & & & $\mathrm{r}$ & & & & h & s & $\mathbf{r}$ & $\mathrm{r}$ & r \\
\hline 69 & Cyprina Islandica Linn. 1767 & . & . & . & - & . & & & $\mathrm{h}$ & s & a & & $\mathrm{h}$ & h & h & $\mathrm{h}$ & h \\
\hline 70 & $\begin{array}{c}\text { Cyprina rustica Sow. } 1818 \text {. } \\
\text { (Cyprina tumida Nyst). }\end{array}$ & - & . & . & · & . & & & $\mathrm{h}$ & $\mathrm{h}$ & a & & & $\mathrm{h}$ & h & s & $s$ \\
\hline
\end{tabular}


$\mathrm{r}=$ auf secundärer Lagerstätte.

$a=$ anwesend, jedoch unbekannt ob häufig oder selten.

\begin{tabular}{|c|c|c|c|c|c|c|c|c|c|c|}
\hline & & & & & & P L E & I S T OC & $\ddot{A} \mathrm{~N}$ & RECEN T & \\
\hline & & BEL & IEN & & & $\begin{array}{r}\text { FAU } \\
\text { (LET }\end{array}$ & $\begin{array}{l}\text { NA DES EE } \\
\text { ZIES INTERGLAC }\end{array}$ & & VORKOMMEN & BFEFYUYAY \\
\hline & I & & & & & NUFDFR= & NORD $=$ & $D \not ̈ N F=$ & IN DER & \\
\hline A & $\begin{array}{l}\text { I } \\
\text { B }\end{array}$ & $\begin{array}{l}\text { H } \\
\mathrm{A}\end{array}$ & $\begin{array}{l}\text { II } \\
\text { B }\end{array}$ & $\begin{array}{l}\text { III } \\
\text { A }\end{array}$ & $\begin{array}{l}\text { III } \\
\text { B }\end{array}$ & LANDE & $\begin{array}{c}\text { DEUTSCH } \\
\text { LAND }\end{array}$ & MARK & HEUTIGEN FAUNA & \\
\hline & h & $\mathrm{s}$ & $\mathrm{r}$ & & & & & & $\begin{array}{l}\text { Westliche Küste Skan= } \\
\text { dinaviens, Shetland=Inseln, } \\
\text { belgische und französische } \\
\text { Küste, Mittelländisches } \\
\text { Meer. In Tiefen von } 8 \\
\text { bis } 180 \mathrm{M} \text {. }\end{array}$ & $\begin{array}{l}\text { Wird von Lorié aus III a } \\
\text { (Amsterdam) erwähnt; sel= } \\
\text { ten in II a der Gegend } \\
\text { Grave } \text { Oss. } \\
\text { Häufig im Miocän. Of= } \\
\text { ters von Cardium fascia= } \\
\text { tum nicht zu unterscheiden. }\end{array}$ \\
\hline $\mathbf{a}$ & & & & & & & & & & $\begin{array}{l}\text { Wird von Lorié aus III a } \\
\text { (Amsterdam, Utrecht) er } \\
\text { wähnt. Diese miocäne Art } \\
\text { findet sich bisweilen in } \\
\text { II a auf secundärer Lager= } \\
\text { stätte. Im Pliocän Englands } \\
\text { und Belgiens unbekannt. } \\
\text { Im Miocän sehr haufig. }\end{array}$ \\
\hline a & $\mathrm{h}$ & $\mathrm{r}$ & & & & & & & $\begin{array}{l}\text { Westküste Norwegens } \\
\text { (Christiansund, Molde, } \\
\text { Sognefjord, Christiania: } \\
\text { fjord), Shetlands, englische } \\
\text { Küsten(Devon, Cornwall), } \\
\text { östlich und südlich von } \\
\text { Irland, westlich von Schott= } \\
\text { land, Arcachon, Mittellän= } \\
\text { disches Meer. Ueberall } \\
\text { local. In Tiefen von } 35 \\
\text { bis } 180 \mathrm{M} \text {. }\end{array}$ & $\begin{array}{l}\text { Von dieser unterpliocä= } \\
\text { nen Art fand ich nur ab= } \\
\text { gerollte Fragmente in IIa } \\
\text { der Gegend Grave=Oss, } \\
\text { offerbar aus dem Ober: } \\
\text { miocän stammend. Beson= } \\
\text { ders in Ib Belgiens häufig, } \\
\text { wie auch im Obermiocän } \\
\text { der nördlichen Peelgegend. }\end{array}$ \\
\hline a & h & $\mathrm{h}$ & $\mathrm{s}$ & & & & a & $a$ & $\begin{array}{l}\text { Östliche Küste Nord= } \\
\text { amerikas, Grönland, Is= } \\
\text { land, nördliche Küste Russ: } \\
\text { lands, Westküste Skandi= } \\
\text { naviens, englische Küsten, } \\
\text { französische Küste (Arca= } \\
\text { chon). Auch bei Helgo= } \\
\text { land. In Tiefen von } 8 \\
\text { bis } 120 \mathrm{M} \text {. }\end{array}$ & $\begin{array}{l}\text { Wird von Lorié aus III a } \\
\text { (Amsterdam,Utrecht, Gor= } \\
\text { kum) erwähnt. Von mir } \\
\text { auch in III (Oudewater, } \\
\text { Barendrecht, Breda) aufge } \\
\text { funden. In II a der Gegend } \\
\text { Grave:Oss häufig. }\end{array}$ \\
\hline $\mathrm{a}$ & $\mathrm{h}$ & $\mathrm{h}$ & $\mathrm{h}$ & & & & & & & $\begin{array}{l}\text { Wird von LoRIE aus III a } \\
\text { (Utrecht) erwähnt. In IIa } \\
\text { und IIb(Grave) sehrhäufig. } \\
\text { Die dünnschaligeForm die } \\
\text { ser Art öfters mit Cyprina } \\
\text { Islandica verwechselt. }\end{array}$ \\
\hline
\end{tabular}


$\mathrm{h}=$,sehr häufig" bis „nicht selten”.

$\mathbf{S}=$ „selten" bis „sehr selten".

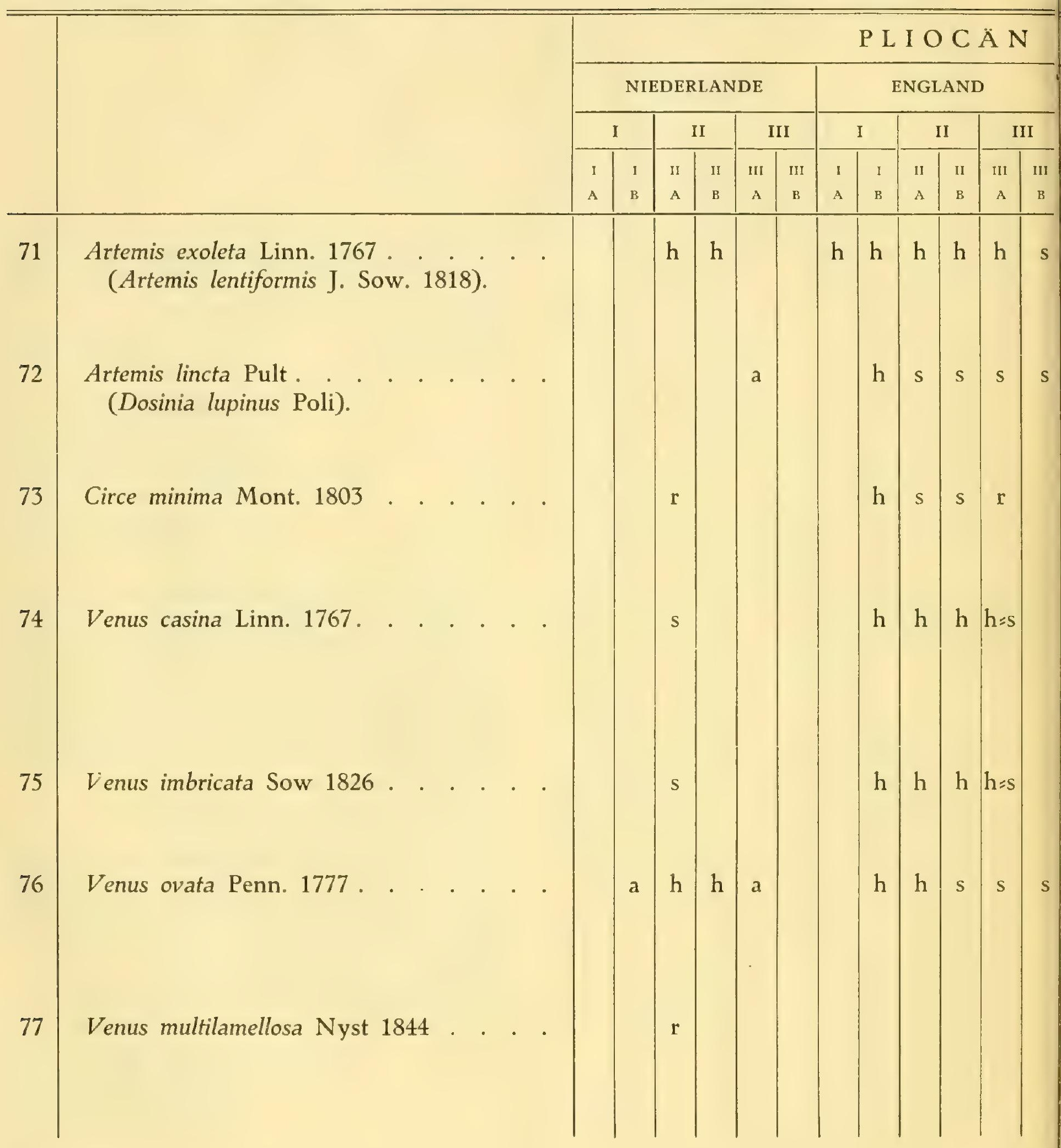


$\mathrm{r}=$ auf secundärer Lagerstätte.

$\mathrm{a}=$ anwesend, jedoch unbekannt ob häufig oder selten.

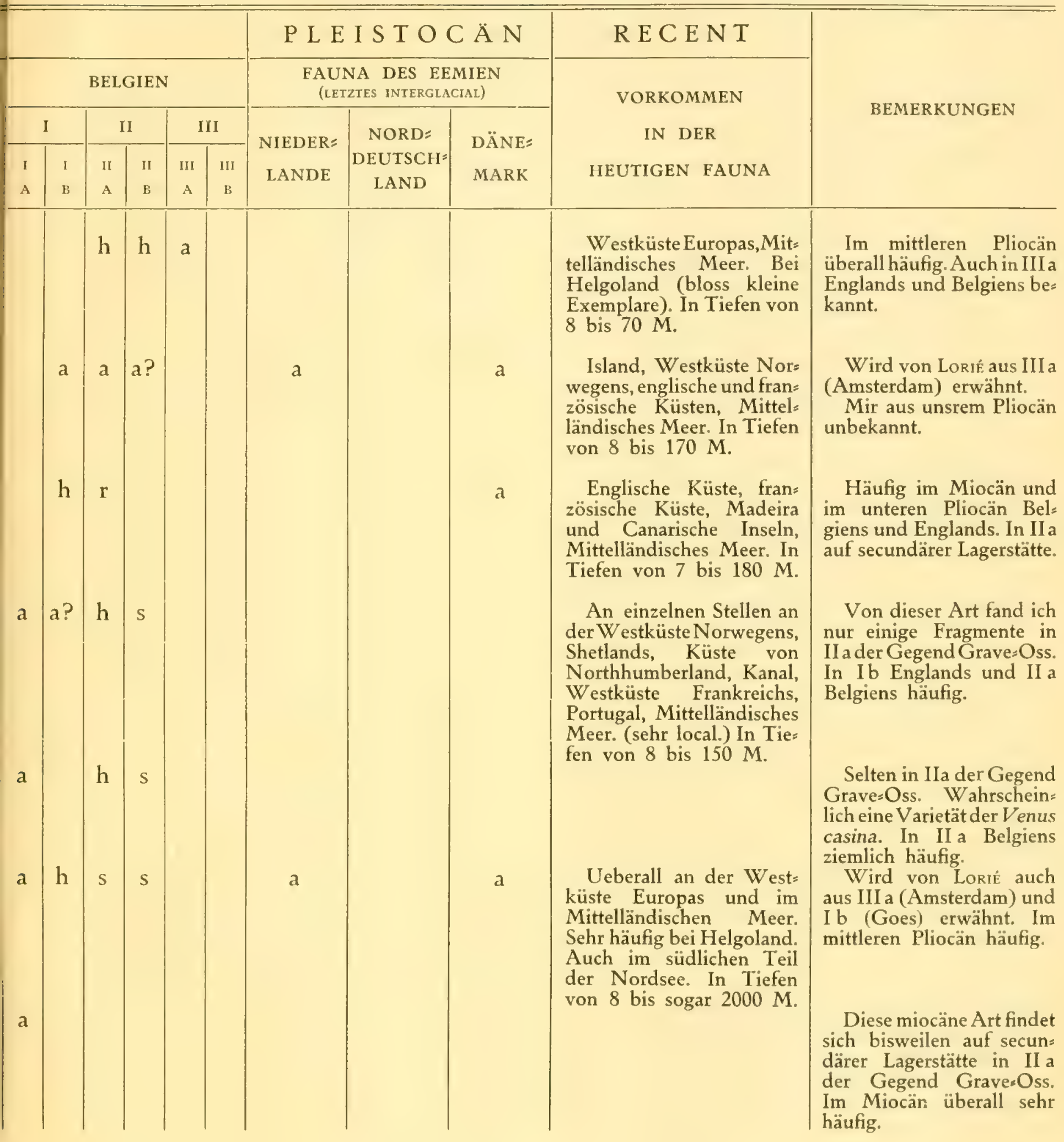


$\mathrm{h}=$ „sehr häufig" bis "nicht selten".

$\mathrm{s}=$ „selten" bis „sehr selten".

\begin{tabular}{|c|c|c|c|c|c|c|c|c|c|c|c|c|c|c|c|c|c|c|}
\hline & & & & & & & \multicolumn{12}{|c|}{ P L I OC $\ddot{A} N$} \\
\hline & & & & & & & \multicolumn{6}{|c|}{ NIEDERLANDE } & \multicolumn{6}{|c|}{ ENGLAND } \\
\hline & & & & & & & & I & 1 & & I & & t. & & 1 & & & II \\
\hline & & & & & & & $\begin{array}{l}\mathrm{I} \\
\mathrm{A}\end{array}$ & $\begin{array}{l}\text { I } \\
\text { B }\end{array}$ & $\begin{array}{l}11 \\
\mathrm{~A}\end{array}$ & $\begin{array}{l}\text { II } \\
\text { B }\end{array}$ & $\begin{array}{c}\text { III } \\
\text { A }\end{array}$ & $\begin{array}{c}\text { III } \\
\text { B }\end{array}$ & I & $\begin{array}{l}1 \\
\text { B }\end{array}$ & $\begin{array}{l}\text { II } \\
\text { A }\end{array}$ & $\begin{array}{l}\text { II } \\
\text { B }\end{array}$ & $\begin{array}{c}\text { III } \\
\text { A }\end{array}$ & $\begin{array}{c}\text { III } \\
\text { B }\end{array}$ \\
\hline 78 & Cytherea Chione Linn. 1767. & - & . & . & - & - & & & $s$ & & & & h & h & & s & s & \\
\hline 79 & Cytherea rudis Polis 1795 . & • & - & . & - & - & & & s & & & & & $\mathrm{h}$ & $\mathrm{h}$ & s & s & \\
\hline 80 & $\begin{array}{c}\text { Tapes edulis Chemn. . } \\
\text { Tapes virginea Chemn.) } \\
\text { nicht Tapes virginea Linn.) }\end{array}$ & . & - & - & . & . & & & s & & & & & s & s & s & s & $s$ \\
\hline 81 & Tapes pullastra Wood. . . & - & . & $\cdot$ & - & . & & a? & a? & & & & & & s & s & s & s \\
\hline 82 & Donax politus Poli 1791 & ${ }^{\circ}$ & . & - & • & . & & & h & & & & h & $\mathrm{h}$ & $s$ & s & $s$ & \\
\hline 83 & $\begin{array}{l}\text { Donax vittatus da Costa } 1778 \\
\text { (Donax anatina Lam.) }\end{array}$ & & . & . & . & • & & & & & $\mathrm{h}$ & & & & & & $s$ & $\mathrm{~s}$ \\
\hline
\end{tabular}


$\mathrm{h}=$ „sehr häufig" bis ,,nicht selten".

$\mathrm{s}=$ „selten” bis „sehr selten".

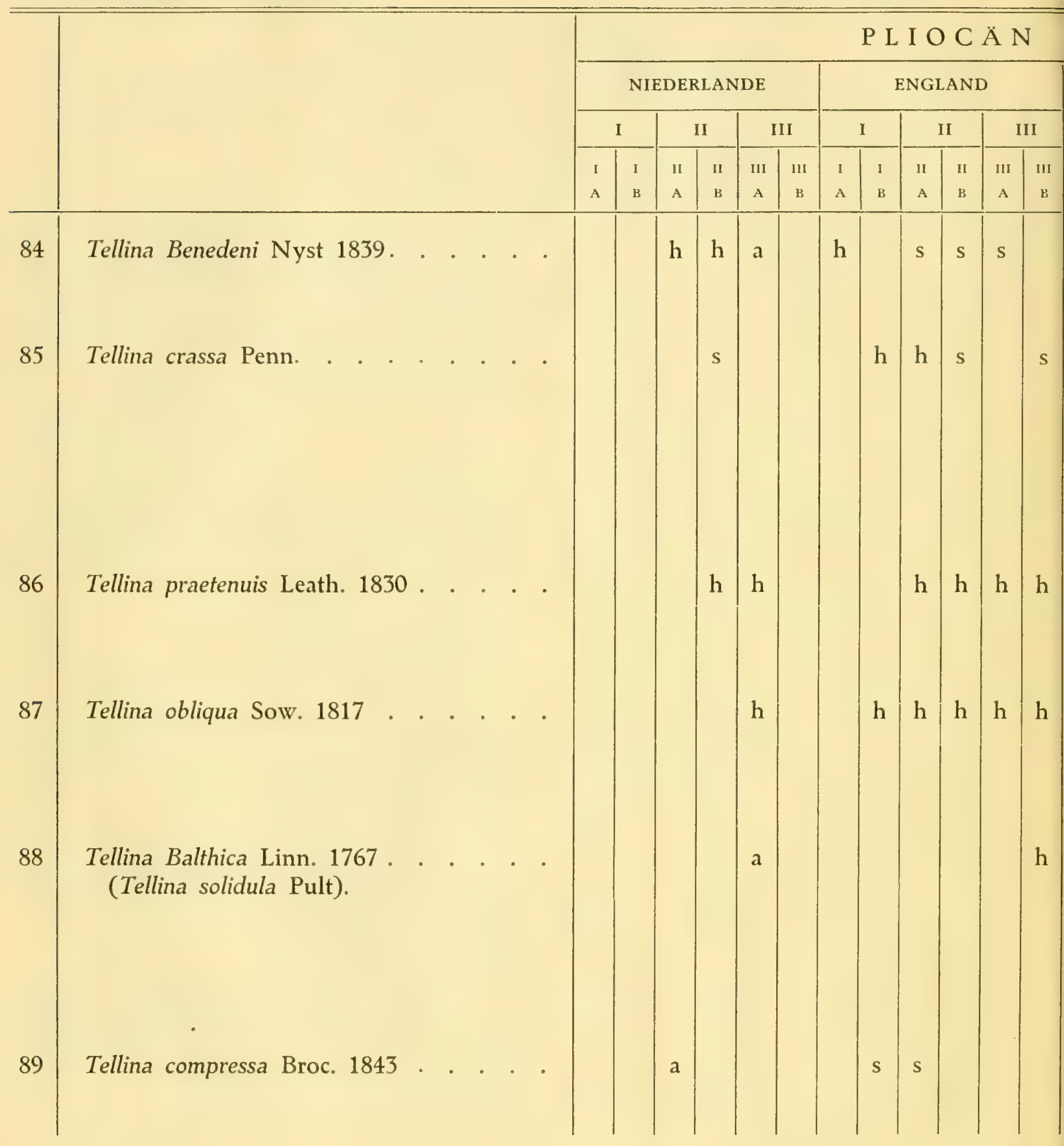


$r=$ auf secundärer Lagerstätte.

$a=$ anwesend, jedoch unbekannt ob häufig oder selten.

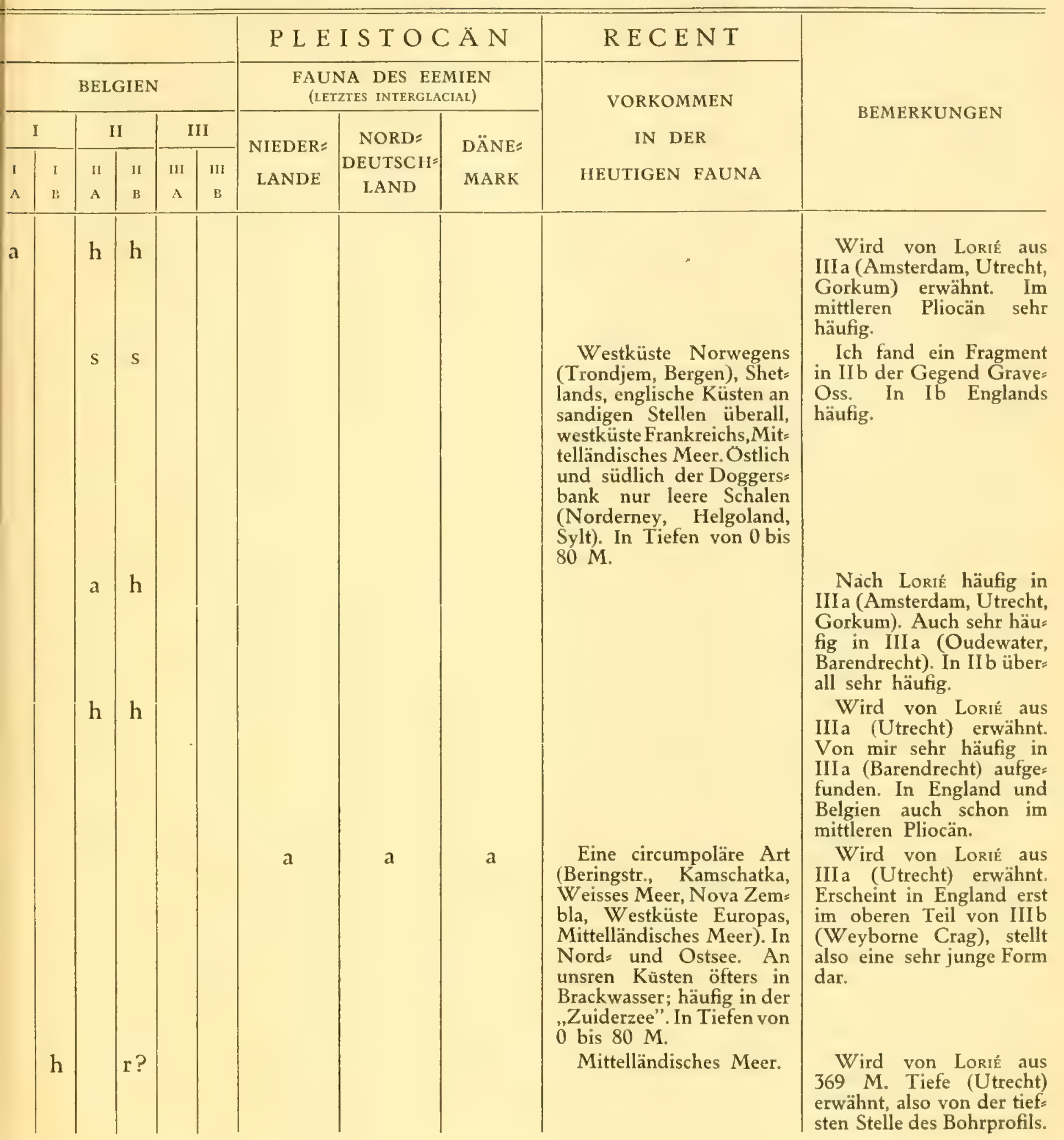


$\mathrm{h}=$,sehr häufig" bis ,nicht selten".

$\mathrm{s}=$ „selten" bis „sehr selten".

\begin{tabular}{|c|c|c|c|c|c|c|c|c|c|c|c|c|c|c|c|c|c|}
\hline & & & & & & \multicolumn{12}{|c|}{ P L I O C $\ddot{A} \mathrm{~N}$} \\
\hline & & & & & & \multicolumn{6}{|c|}{ NIEDERLANDE } & \multicolumn{6}{|c|}{ ENGLAND } \\
\hline & & & & & & \multicolumn{2}{|c|}{ I } & \multicolumn{2}{|c|}{ II } & \multicolumn{2}{|c|}{ III } & \multicolumn{2}{|c|}{ I } & \multicolumn{2}{|c|}{ II } & \multicolumn{2}{|c|}{ III } \\
\hline & & & & & & $\begin{array}{l}\text { I } \\
\text { A }\end{array}$ & $\begin{array}{l}\mathrm{I} \\
\mathrm{B} \\
\end{array}$ & $\begin{array}{l}\text { II } \\
\text { A }\end{array}$ & $\begin{array}{l}\text { II } \\
\text { B }\end{array}$ & $\begin{array}{c}\text { III } \\
\text { A }\end{array}$ & $\begin{array}{c}\text { III } \\
\text { B }\end{array}$ & $\begin{array}{l}\mathrm{I} \\
\mathrm{A}\end{array}$ & $\begin{array}{l}\text { I } \\
\text { B }\end{array}$ & $\begin{array}{l}\text { II } \\
\text { A }\end{array}$ & $\begin{array}{l}\text { II } \\
\text { B }\end{array}$ & $\begin{array}{l}\text { III } \\
\text { A }\end{array}$ & II \\
\hline 90 & Tellina donacina Linn. 1767. & .. & . & . & . & & & a & h & & & h & $\mathrm{h}$ & s & & & \\
\hline 91 & $\begin{array}{c}\text { Gastrana laminosa Sow. } 1827 \\
\text { (Gastrana fragilis Linn.?) } \\
\text { Petricola lamellosa Lam.? }\end{array}$ & . & . & - & - & & & a & a & & & & h & $\mathrm{h}$ & h & $h=s$ & \\
\hline 92 & Psammobia ferröensis Chemn. & 1782 & . & - & • & & & a & a & & & & h & s & $\mathbf{s}$ & $\mathrm{s}$ & \\
\hline 93 & Psammobia vespertina Chemn. & 1782 & - & - & . & & a & & a & & & & s & & & & \\
\hline 94 & Psammobia tellinella Lam. 1818 & 8 & - & - & • & & & $\mathbf{s}$ & & & & & $\mathrm{h}$ & & & & \\
\hline 95 & Psammobia costulata Turton. & . & - & . & • & & & $s$ & & & & & s & & & & \\
\hline
\end{tabular}


$\mathrm{r}=$ aut secundärer Lagerstätte.

$a=$ anwesend, jedoch unbekannt ob häufig oder selten.

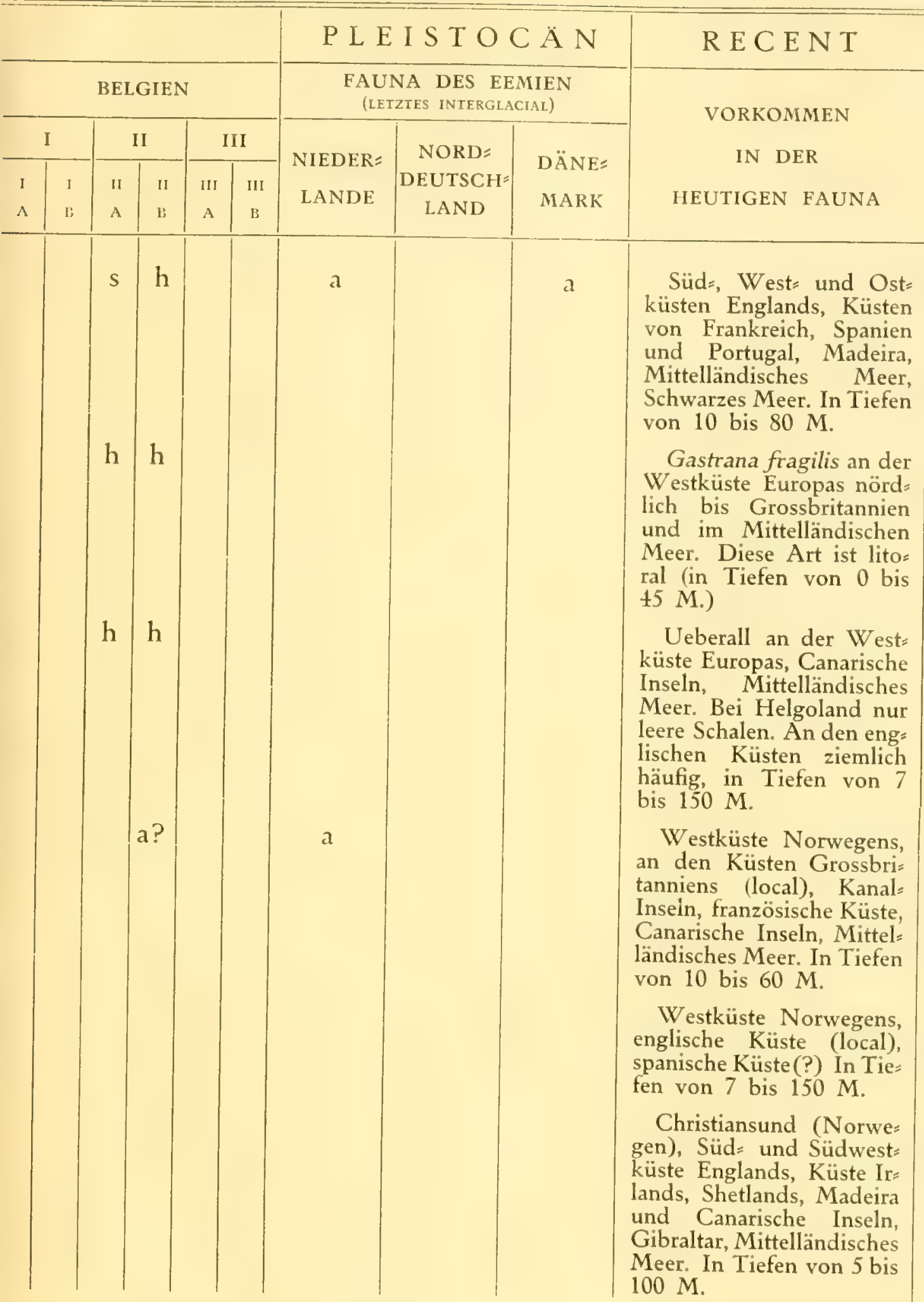

BEMERKUNGEN

In II b der Gegend Grave=Oss sehr häufig. Ebenso häufig in Ib Eng. lands und II b Belgiens.

Nicht sehr häufig in IIa und II b der Gegend Grave= Oss. Meistens nur Frag= mente.

Nicht häufig und meis: tens nur Fragmente. Wird von Lorí́ aus II a (Goes) erwähnt.

Kleine Exemplare, wel= che vielleicht $z u$ dieser Art gehören, wurden von Lorié aus Ib (Goes) und IIb (Utrecht) erwähnt.

Selten in IIa der Ge= gend Grave=Oss.

Ich fand ein einziges Exemplar in II a der Ge= gend Grave=Oss. 
$\mathrm{h}=$ „sehr häufig" bis ,nicht selten".

$\mathrm{s}=$,selten" bis "sehr selten".

\begin{tabular}{|c|c|c|c|c|c|c|c|c|c|c|c|c|c|c|c|c|c|c|}
\hline & & & & & & & \multicolumn{12}{|c|}{$\mathrm{PLIOC} \ddot{\mathrm{A}} \mathrm{N}$} \\
\hline & & & & & & & \multicolumn{6}{|c|}{ NIEDERLANDE } & \multicolumn{6}{|c|}{ ENGLAND } \\
\hline & & & & & & & \multicolumn{2}{|c|}{1} & \multicolumn{2}{|c|}{ II } & \multicolumn{2}{|c|}{ III } & \multicolumn{2}{|c|}{ I } & \multicolumn{2}{|c|}{ II } & \multicolumn{2}{|c|}{ III } \\
\hline & & & & & & & I & $\begin{array}{l}\text { I } \\
\mathrm{B}\end{array}$ & $\begin{array}{l}\text { " } \\
\mathrm{A}\end{array}$ & II & AII & mil & $\begin{array}{l}\mathrm{I} \\
\mathrm{A}\end{array}$ & $\begin{array}{l}\mathrm{I} \\
\mathrm{B}\end{array}$ & II & $\begin{array}{l}\text { II } \\
\text { B }\end{array}$ & A & \\
\hline 96 & Solecurtus strigillatus Linn. 176 & & • & - & . & . & & & s & & & & & s & & & & \\
\hline 97 & Ensis ensis Linn. 1767. & . & . & . & · & . & & a & h & h & $\mathrm{h}$ & & $\mathrm{h}$ & $\mathrm{h}$ & $\mathrm{h}$ & $\mathrm{h}$ & $\mathrm{h}$ & \\
\hline 98 & $\begin{array}{l}\text { Solen siliqua Linn. } 1767 \text {. } \\
\text { var. gladiolus Gray. } 1839\end{array}$ & . & . & . & . & . & & & $\mathrm{h}$ & $\mathrm{h}$ & h & & & & h & $\mathrm{h}$ & h & \\
\hline 99 & $\begin{array}{l}\text { Semele alba Wood } 1802 . \\
\text { (Syndosmya alba Wood. } \\
\text { Abra alba Wood. } \\
\text { Ligula alba Wood). }\end{array}$ & . & . & . & . & . & & & $\mathbf{s}$ & $\mathrm{s}$ & $\mathrm{s}$ & & & $\mathrm{h}$ & $\mathrm{h}$ & h & $\mathrm{s}$ & \\
\hline 100 & Semele prismatica Mont. 1808 & . & • & . & . & . & & & $\mathrm{h}$ & $\mathrm{h}$ & & & & $s$ & $s$ & & & \\
\hline 101 & Semele fabalis Wood 1848 . & $\cdot$ & $\cdot$ & . & . & . & & & & & $a$ & & & & $\mathrm{~s}$ & S & $\mathrm{s}$ & \\
\hline
\end{tabular}


$\mathrm{h}=$ "sehr häufig" bis "nicht selten".

$\mathrm{S}=$ "selten" bis ,sehr selten".

\begin{tabular}{|c|c|c|c|c|c|c|c|c|c|c|c|c|c|c|c|c|c|}
\hline & & & & & & \multicolumn{12}{|c|}{$\mathrm{PLIOC} \ddot{\mathrm{A}} \mathrm{N}$} \\
\hline & & & & & & \multicolumn{6}{|c|}{ NIEDERLANDE } & \multicolumn{6}{|c|}{ ENGLAND } \\
\hline & & & & & & \multicolumn{2}{|r|}{ I } & \multicolumn{2}{|c|}{ II } & \multicolumn{2}{|c|}{ III } & \multicolumn{2}{|c|}{ I } & \multicolumn{2}{|c|}{ II } & \multicolumn{2}{|c|}{ III } \\
\hline & & & & & & A & $\begin{array}{l}1 \\
\text { B }\end{array}$ & A & II & \begin{tabular}{c|} 
III \\
A
\end{tabular} & $\begin{array}{ll}\text { III } \\
\text { B }\end{array}$ & I & $\begin{array}{l}1 \\
\text { B }\end{array}$ & A & II & AII & $\begin{array}{l}\text { III } \\
\text { b }\end{array}$ \\
\hline 102 & $\begin{array}{l}\text { Scrobicularia piperata Gmelin } 178 \\
\text { (Trigonella plana da Costa } 177\end{array}$ & $\begin{array}{l}88 \\
78) .\end{array}$ & & . & . & & & & ? & a & & & & & & s & $h=s$ \\
\hline 103 & Mactra stultorum Linn. 1767 & . & - & $\cdot$ & . & & & & & a & & & s & s & & s & S \\
\hline 104 & Mactra arcuata Sow. 1817 & - & • & · & $\cdot$ & & & $\mathrm{h}$ & $\mathrm{h}$ & . & & $\mathrm{h}$ & $\mathrm{h}$ & $\mathrm{h}$ & $\mathrm{h}$ & $h=s$ & S \\
\hline 106 & $\begin{array}{l}\text { Mactra ovalis Sow } 1817 . \\
\text { (Mactra elliptica Brown. 1827). } \\
\text { Mactra dubia Sow. 1817. } \\
\text { Mactra striata Nyst 1844). } \\
\text { Mactra solida Linn. 1766. }\end{array}$ & . & • & . & . & & & & $\mathrm{h}$ & $\mathrm{h}$ & & & & & & $\mathrm{h}$ & - \\
\hline 106 & Mactra solida Linn. 1766. & - & • & $\cdot$ & - & & a? & a? & $\mathrm{s}$ & $\mathbf{s}$ & & & $\mathbf{s}$ & s & $\mathrm{s}$ & $s=h$ & h \\
\hline
\end{tabular}


$\mathrm{h}=$ „sehr häufig" bis "nicht selten".

$\mathrm{s}=$,selten" bis „sehr selten".

\begin{tabular}{|c|c|c|c|c|c|c|c|c|c|c|c|c|c|c|c|}
\hline & & & & & \multicolumn{11}{|c|}{ P L I O C $\ddot{A} N$} \\
\hline & & & & & \multicolumn{6}{|c|}{ NIEDERLANDE } & \multicolumn{5}{|c|}{ ENGLAND } \\
\hline & & & & & \multicolumn{2}{|c|}{ I } & \multicolumn{2}{|c|}{ II } & \multicolumn{2}{|c|}{ III } & \multicolumn{2}{|c|}{ I } & \multicolumn{2}{|c|}{ II } & III \\
\hline & & & & & $\begin{array}{l}\mathrm{I} \\
\mathrm{A}\end{array}$ & $\begin{array}{l}\text { I } \\
\text { B }\end{array}$ & $\begin{array}{l}\text { II } \\
\text { A }\end{array}$ & $\begin{array}{l}\text { II } \\
\text { B }\end{array}$ & $\begin{array}{l}\mathrm{III} \\
\mathrm{A}\end{array}$ & $\begin{array}{l}\text { III } \\
\text { B }\end{array}$ & $\begin{array}{l}\text { I } \\
\text { A }\end{array}$ & $\begin{array}{l}\text { I } \\
\text { B }\end{array}$ & $\begin{array}{l}\text { II } \\
\text { A }\end{array}$ & $\begin{array}{l}\text { II } \\
\text { B }\end{array}$ & \\
\hline 107 & $\begin{array}{l}\text { Mactra subtruncata da Costa } 1778 \\
\text { (Mactra triangula Ren.) }\end{array}$ & • & - & : & & a & $\mathrm{h}$ & $\mathrm{h}$ & $\mathrm{h}$ & & & & & & \\
\hline 108 & Mactra obtruncata Wood 1850. & • & - & . & & & $a$ & $\mathrm{a}$ & & & & $\mathrm{h}$ & h & h & \\
\hline 109 & Mactra deaurata Turton 1822 . & . & . & . & & & a & h & $\mathrm{h}$ & & & & s & s & \\
\hline 110 & Panopaea Faujasi Mén. 1807 . . & • & . & • & & & a & & & & & $\mathrm{h}$ & $\mathrm{h}$ & h & $:$ \\
\hline 111 & $\begin{array}{l}\text { Glycimeris siliqua Chemn. . } \\
\text { (Glycimeris angusta Nyst 1839). }\end{array}$ & . & - & . & & & & h & $\mathrm{a}$ & & & h & & a & \\
\hline
\end{tabular}




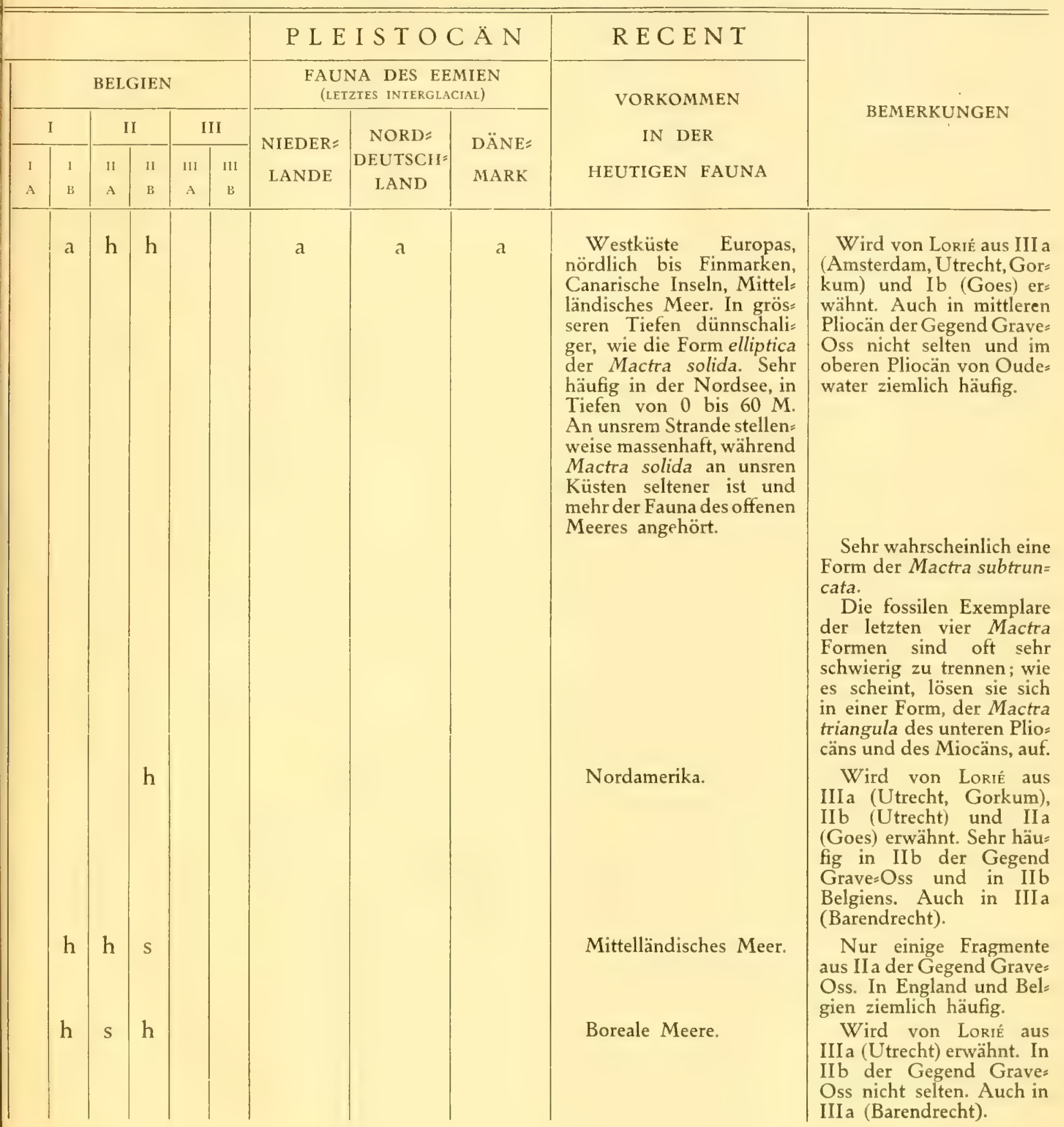


$\mathrm{h}=$ „sehr häufig" bis „nicht selten”.

$\mathrm{s}=$ „selten" bis „sehr selten".

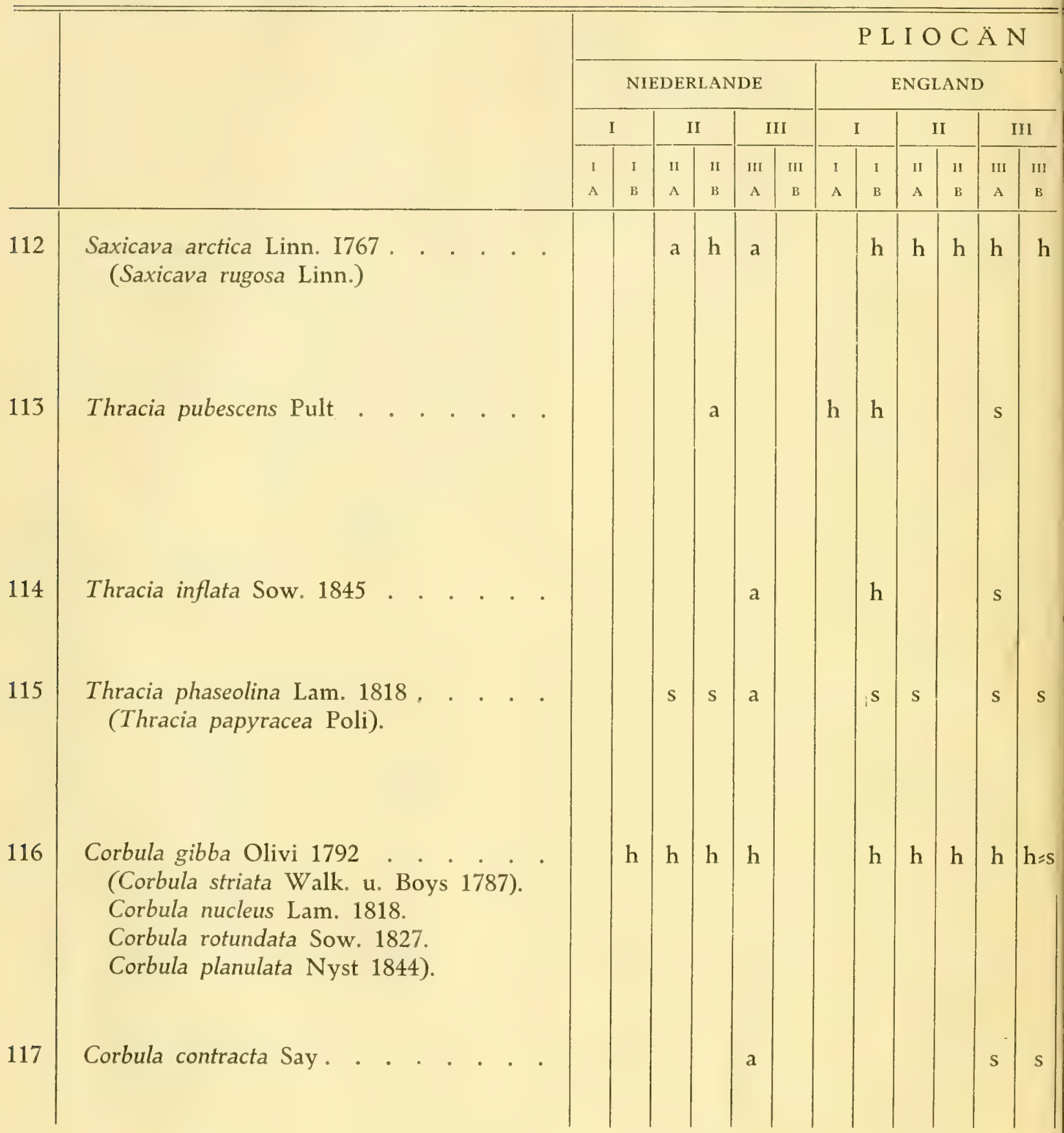


$a=$ anwesend, jedoch unbekannt ob häufig oder selten.

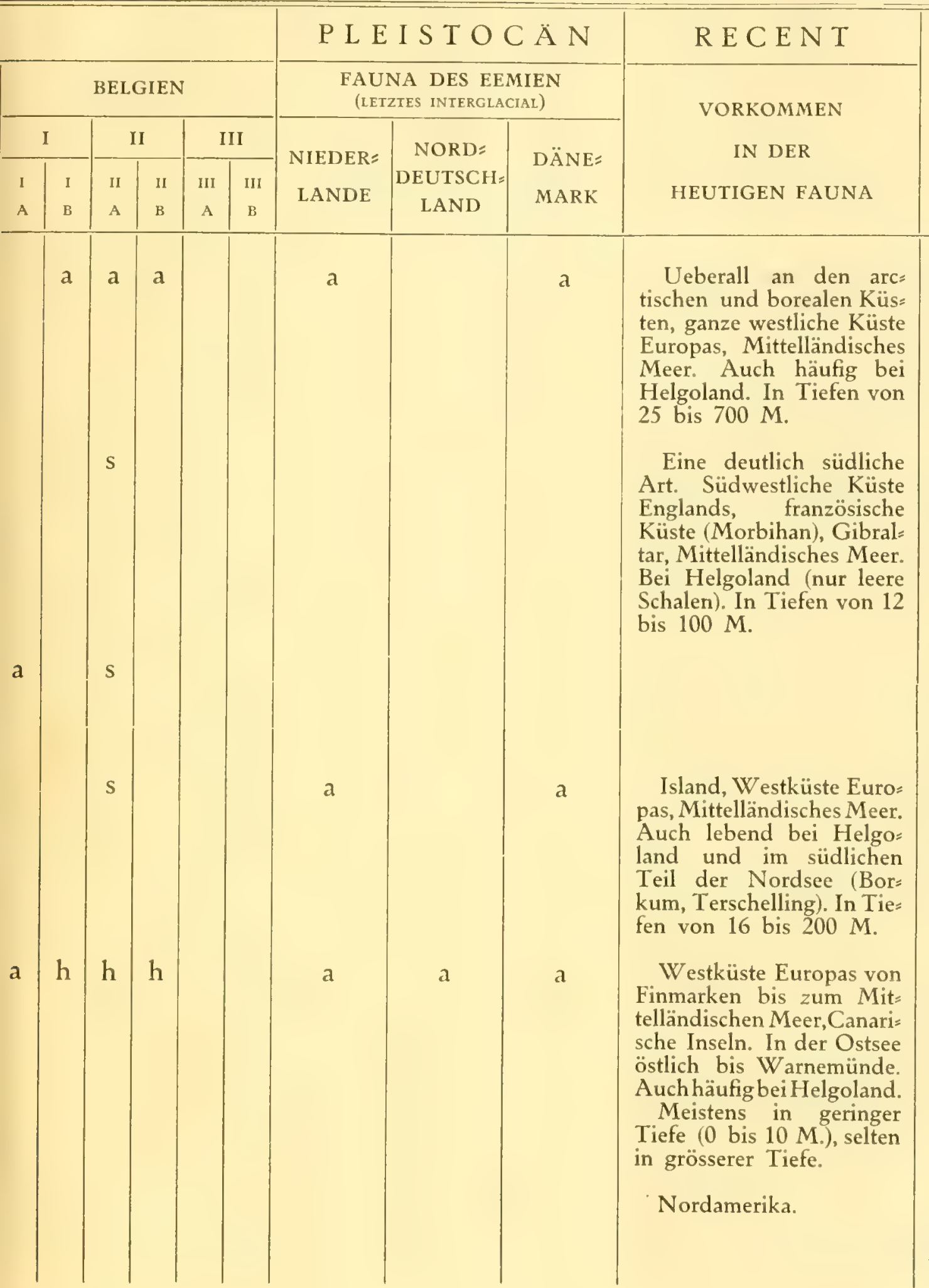

Wird von LorIÉ aus III a (Amsterdam) erwähnt (as $S$. rugosa). In II b der Gegend Grave=Oss häufig.

Von LoRIÉ aus IIb (Utrecht) erwähnt. Mir aus unsrem Boden nicht be= kannt.

Von LorIÉ aus III a (Amsterdam) erwähnt. Mir aus unsrem Boden nicht bekannt.

Von Lorié aus IIIa (Amsterdam) erwähnt. Auch in II a und IIb der Gegend Grave:Oss. Scheint überall selten.

Ueberall sehr häufig.

Wird von LorIÉ aus III a (Amsterdam) erwähnt. Mir aus unsrem Boden unbekannt. 
$\mathrm{h}=$ „sehr häufig" bis „nicht selten”.

$\mathrm{S}=$ "selten" bis "sehr selten".

\begin{tabular}{|c|c|c|c|c|c|c|c|c|c|c|c|c|c|c|c|c|c|}
\hline & & & & & & \multicolumn{12}{|c|}{ P L I OC $\ddot{A} N$} \\
\hline & & & & & & \multicolumn{6}{|c|}{ NIEDERLANDE } & \multicolumn{6}{|c|}{ ENGLAND } \\
\hline & & & & & & \multicolumn{2}{|c|}{ I } & \multicolumn{2}{|c|}{ II } & \multicolumn{2}{|c|}{ III } & \multicolumn{2}{|c|}{ I } & \multicolumn{2}{|c|}{ II } & \multicolumn{2}{|c|}{ III } \\
\hline & & & & & & $\begin{array}{l}1 \\
\mathrm{~A}\end{array}$ & $\begin{array}{l}\text { I } \\
\text { B }\end{array}$ & $\begin{array}{l}\mathrm{II} \\
\mathrm{A}\end{array}$ & $\begin{array}{l}\text { II } \\
\text { B }\end{array}$ & $\begin{array}{l}111 \\
\mathrm{~A}\end{array}$ & $\begin{array}{c}\text { III } \\
\text { в }\end{array}$ & I & $\begin{array}{l}\text { I } \\
\text { E }\end{array}$ & $\begin{array}{l}\mathrm{II} \\
\mathrm{A}\end{array}$ & $\begin{array}{l}\text { II } \\
\text { B }\end{array}$ & $\begin{array}{l}\text { III } \\
\text { A }\end{array}$ & \\
\hline 118 & Corbulomya complanata Sow. & 1822 & & & . & & & & $\mathrm{h}$ & & & & & $\mathrm{h}$ & h & $h=s$ & \\
\hline 119 & $\begin{array}{l}\text { Poromya granulata Nyst } 1839 \\
\text { (Embla Koreni Lovén). }\end{array}$ & . & & & $\cdot$ & & & $a$ & & & & & $\mathrm{~s}$ & $\mathrm{~s}$ & $\mathrm{~s}$ & & \\
\hline 120 & Mya arenaria Linn. 1767. & .. & & & . & & & & & $\mathrm{h}$ & & & & $\mathrm{h}$ & $\mathrm{h}$ & h & \\
\hline 121 & Mya truncata Linn. 1767. & . . & & & , & & & $\mathrm{h}$ & h & a & & & $\mathrm{h}$ & h & s & h & \\
\hline 122 & $\begin{array}{l}\text { Mya Binghami Turton } 1822 . \\
\text { (Sphenia Binghami Turton } \\
\text { Mya fragilis Nyst 1881). }\end{array}$ & 1822 & & & 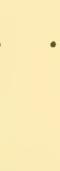 & & & $a$ & $a$ & $a$ & & & $\mathrm{~s}$ & $\mathbf{s}$ & $s$ & & \\
\hline 123 & $\begin{array}{l}\text { Pholas crispata Linn. } 1767 \\
\text { (Zirphaea crispata Linn.) }\end{array}$ & $\cdot$ & & & . & & & $a$ & & & & & & h & h & h & \\
\hline
\end{tabular}


$\mathrm{h}=$,sehr häufig" bis ,nicht selten”.

$\mathrm{s}=$ "selten" bis "sehr selten".

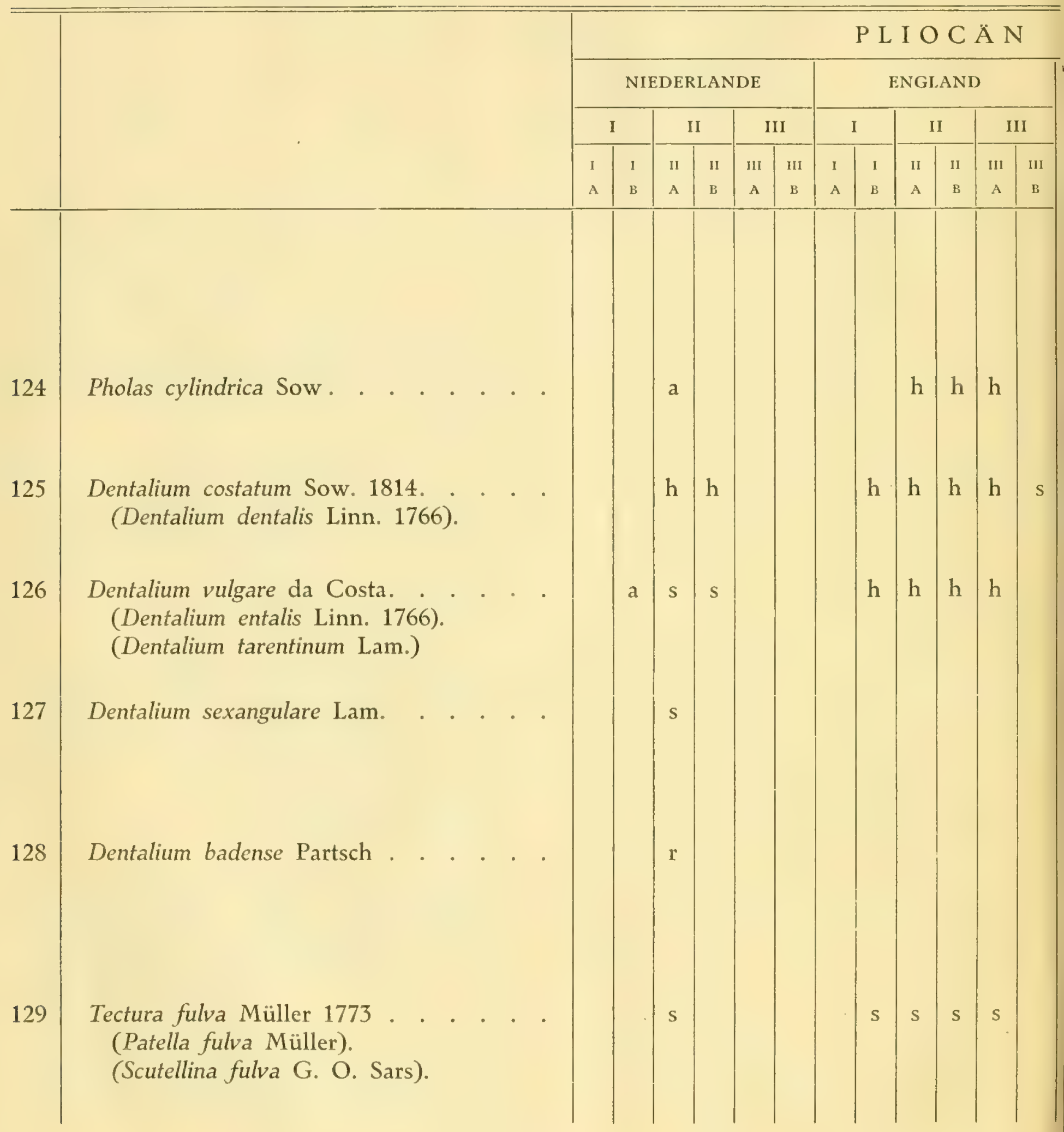




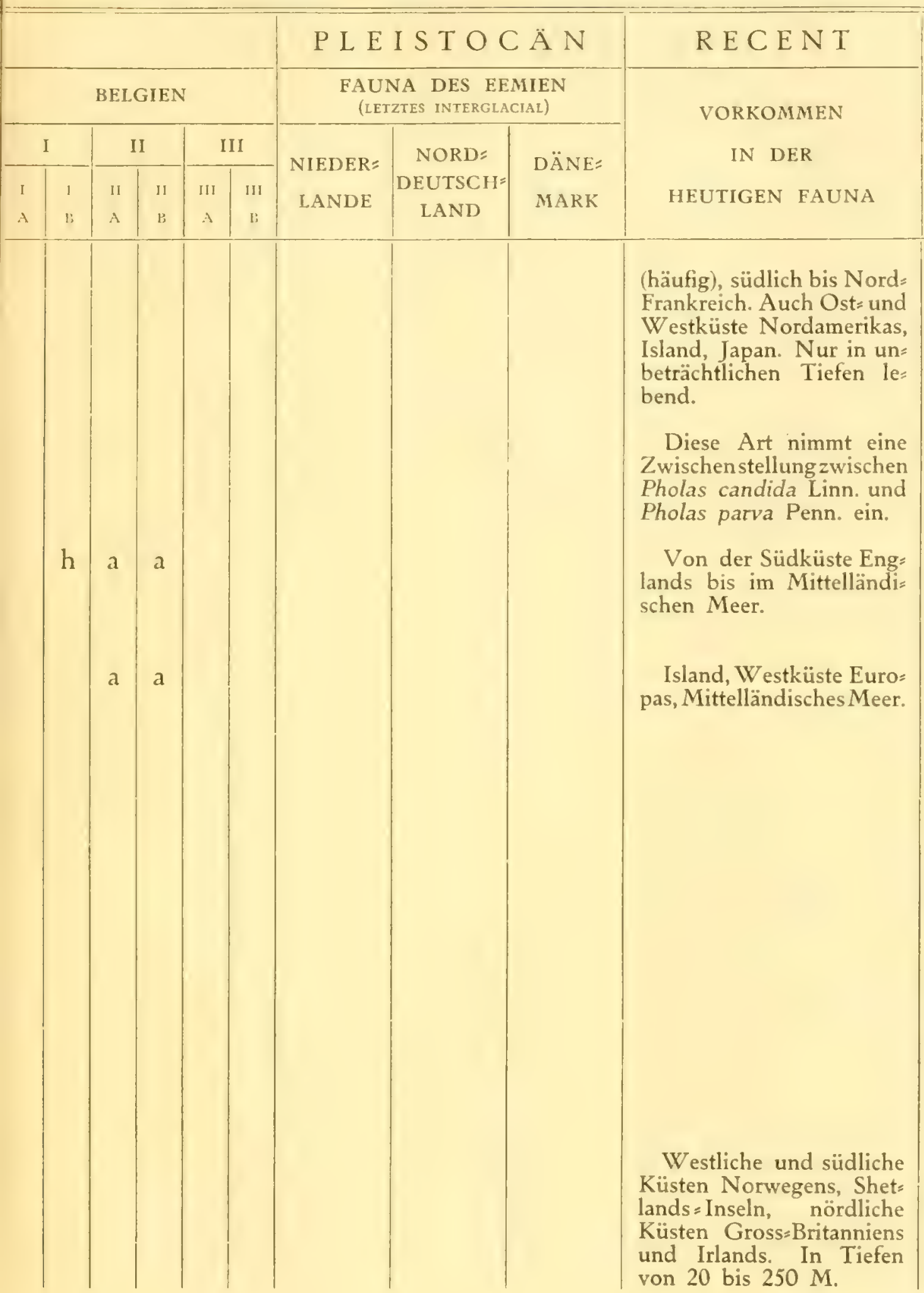

BEMERKUNGEN

Ein einziges Exemplar aus II a der Gegend Grave= Oss.

Im mittleren Pliocän der Gegend Grave=Oss nicht selten. Auch aus II a (Utrecht) bekannt.

Im mittleren Pliocän der Gegend Grave=Oss ziem= lich selten. Auch aus II a und IIb (Utrecht) und Ib (Goes) bekannt.

Ein einziges Exemplar aus II a der Gegend Grave= Oss. Aus dem Pliocän Italiens bekannt. Aus Eng: land und Belgien nicht erwähnt.

Diese miocäne Form kommt in der Gegend Grave $=$ Oss an der Basis des Pliocäns. vereinzelt auf secundärer Lagerstätte vor. Im oberen Miocän dieser Gegend häufig.

Ein einziges Exemplar aus II a der Gegend Grave= Oss. Aus Belgien vielleicht als Lepeta erwähnt. 
$\mathrm{h}=$,sehr häufig" bis „nicht selten”.

$\mathrm{s}=$,selten" bis ,sehr selten".

\begin{tabular}{|c|c|c|c|c|c|c|c|c|c|c|c|c|c|c|c|c|c|}
\hline & & & & & & \multicolumn{12}{|c|}{ P L I O C $\ddot{A} \mathrm{~N}$} \\
\hline & & & & & & \multicolumn{6}{|c|}{ NIEDERLANDE } & \multicolumn{6}{|c|}{ ENGLAND } \\
\hline & & & & & & \multicolumn{2}{|c|}{1} & \multicolumn{2}{|c|}{ II } & \multicolumn{2}{|c|}{ III } & \multicolumn{2}{|c|}{ I } & \multicolumn{2}{|c|}{ II } & \multicolumn{2}{|c|}{ III } \\
\hline & & & & & & A & I & A & ${ }_{\mathrm{B}}^{11}$ & AII & BII & A & ${ }_{\text {в }}^{1}$ & II & $\begin{array}{l}\text { II } \\
\text { B }\end{array}$ & $\mathrm{A}$ & III \\
\hline 130 & $\begin{array}{l}\text { Emarginula fissura Linn. } 1767 \\
\text { (Emarginula reticulata Sow.) }\end{array}$ & - & 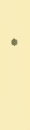 & . & . & & s & & & & & h & h & h & $\mathrm{h}$ & $h=s$ & \\
\hline 131 & $\begin{array}{l}\text { Trochus cineröides Wood } 1842 . \\
\text { (Trochus solarium Nyst 1844). }\end{array}$ & . & - & . & . & & $\mathrm{s}$ & s & $\mathrm{h}$ & & & & & h & $\mathrm{h}$ & $h=s$ & \\
\hline 132 & Trochus zizyphinus Linn. 1767. & • & - & - & - & & a? & & & & & $\mathrm{h}$ & $\mathrm{h}$ & $\mathrm{h}$ & $\mathrm{h}$ & h & s \\
\hline 133 & Xenophora Deshayesi Mich . . & - & - & - & . & & & h & & & & & & & & & \\
\hline 134 & Adeorbis subcarinatus Mont. . & - & - & - & - & & & s & & & & & $\mathrm{h}$ & s & s & s & \\
\hline 135 & Littorina littorea Linn. 1766. & • & - & - & . & & & & & $\mathrm{h}$ & & & & & & $\mathrm{h}$ & h \\
\hline 136 & Littorina suboperta Sow. 1813 . & $\bullet$ & • & - & - & & & & h & & & & & h & $\mathrm{h}$ & $h=s$ & \\
\hline
\end{tabular}


$h=$ „sehr häufig” bis ,nicht selten”.

$\mathrm{s}=$ „selten" bis „sehr selten”.

\begin{tabular}{|c|c|c|c|c|c|c|c|c|c|c|c|c|c|c|c|c|}
\hline & & & & & \multicolumn{12}{|c|}{ P L I O C $A$ N } \\
\hline & & & & & \multicolumn{6}{|c|}{ NIEDERLANDE } & \multicolumn{6}{|c|}{ ENGLAND } \\
\hline & & & & & \multicolumn{2}{|c|}{ I } & \multicolumn{2}{|c|}{ II } & \multicolumn{2}{|c|}{ III } & \multicolumn{2}{|c|}{ I } & \multicolumn{2}{|c|}{ II } & \multicolumn{2}{|c|}{ III } \\
\hline & & & & & $\begin{array}{l}\text { I } \\
\text { A }\end{array}$ & I & $\begin{array}{l}\text { II } \\
\text { A }\end{array}$ & $\begin{array}{l}11 \\
\mathrm{~B}\end{array}$ & $\begin{array}{c}\text { III } \\
\text { A }\end{array}$ & $\begin{array}{l}\text { III } \\
\text { B }\end{array}$ & A & I & $\begin{array}{l}11 \\
\mathrm{~A}\end{array}$ & $\begin{array}{l}\text { II } \\
\text { B }\end{array}$ & $\begin{array}{c}\mathrm{III} \\
\mathrm{A}\end{array}$ & $\begin{array}{c}\text { III } \\
\text { B }\end{array}$ \\
\hline 137 & Littorina terebellata Nyst 1844. & . & - & - & & & & $\mathrm{h}$ & $\mathrm{a}$ & & & & $\mathrm{h}$ & $\mathrm{h}$ & $\mathrm{h}$ & \\
\hline 138 & Littorina pendula Wood 1842 . . & . & . & . & & & & s & & & & & $\mathrm{h}$ & $\mathrm{h}$ & $h=s$ & \\
\hline 139 & Capulus ungaricus Linn. 1767 . . & . & . & . & & a & $\mathrm{h}$ & $\mathrm{h}$ & & & & h & $\mathrm{h}$ & $\mathrm{h}$ & $\mathrm{h}$ & S \\
\hline 140 & $\begin{array}{l}\text { Capulus recurvatus Wood } 1842 \\
\text { (Capulus ungaricus var. sinuosus }\end{array}$ & $\dot{\mathrm{Br}}$ & ic) & $\cdot$ & & a & & & & & & $\mathrm{h}$ & $\mathrm{h}$ & s & s & \\
\hline 141 & Calyptraea Chinensis Linn. 1767 . & . & . & . & & a & $\mathrm{h}$ & $\mathrm{h}$ & $a$ & & & $\mathrm{~h}$ & $\mathrm{~h}$ & $\mathrm{~h}$ & $\mathrm{~h}$ & S \\
\hline 142 & $\begin{array}{l}\text { Natica catena da Costa } 1778 \\
\text { (Natica monilifera Lam. 1822). }\end{array}$ & . & - & - & & & $\mathrm{h}$ & $\mathrm{h}$ & $\mathrm{h}$ & & & s & $\mathbf{S}$ & $\mathbf{S}$ & $\mathrm{h}$ & \\
\hline
\end{tabular}


$\mathrm{a}=$ anwesend, jedoch unbekannt ob häufig oder selten.

\begin{tabular}{|c|c|c|c|c|c|c|c|c|c|}
\hline & & & & & & P L E & I S TOC & $\ddot{A} \mathrm{~N}$ & R E C E N T \\
\hline & & BEL & IEN & & & $\underset{(\mathrm{IE})}{\mathrm{FAU}}$ & $\begin{array}{l}\text { NA DES EE } \\
\text { ZTES INTERGLA }\end{array}$ & $\begin{array}{l}\text { IIEN } \\
\text { IAL) }\end{array}$ & VORKOMMEN \\
\hline & & & & & & NIFDER= & NORD= & $D \ddot{A N E}=$ & IN DER \\
\hline $\begin{array}{l}\mathrm{I} \\
\mathrm{A}\end{array}$ & $\begin{array}{l}\text { I } \\
\text { B }\end{array}$ & II & $\begin{array}{l}\text { II } \\
\text { B }\end{array}$ & $\begin{array}{l}\text { III } \\
\text { A }\end{array}$ & $\begin{array}{c}\text { III } \\
\text { B }\end{array}$ & LANDE & $\begin{array}{c}\text { DEUTSCH } \\
\text { LAND }\end{array}$ & MARK & HEUTIGEN FAUNA \\
\hline & & s & $\mathrm{h}$ & & & & & & \\
\hline & $a$ & $\mathrm{~h}$ & $\mathrm{~h}$ & & & & & & $\begin{array}{l}\text { Westliche Küsten Euro= } \\
\text { pas vom Nordkap bis Gi= } \\
\text { braltar, ostliche Küsten } \\
\text { Nordamerikas. Wird im } \\
\text { Süden grösser als im Nor= } \\
\text { den. In Tiefen von } 18 \text { bis } \\
180 \mathrm{M} \text {. }\end{array}$ \\
\hline$a$ & $\mathrm{~h}$ & $\mathrm{~h}$ & $\mathrm{~h}$ & & & & & & $\begin{array}{l}\text { Mittelländisches Meer, } \\
\text { Kanarische Inseln, spani= } \\
\text { sche und französische Küs= } \\
\text { ten, nördlich bis Süd=Eng= } \\
\text { land. In Tiefen von } 0 \text { bis } \\
100 \mathrm{M} \text {. }\end{array}$ \\
\hline & & $\mathrm{h}$ & $\mathrm{h}$ & & & & & & $\begin{array}{l}\text { Westliche Küsten Euro: } \\
\text { pas von Süd=Schweden bis } \\
\text { zum Mittelländischen Meer } \\
\text { In Seichtwasser (bis } 18 \mathrm{M} \text {. } \\
\text { Tiefe). Nicht in arctischen } \\
\text { Regionen. }\end{array}$ \\
\hline
\end{tabular}

BEMERKUNGEN

Häufig in IIb der Ge= gend Grave=Oss, aber im= mer sehr local auftretend. Wird von LoRIÉ auch aus IIIa (Gorkum) erwähnt; von mir in III a (Oude: water, Barendrecht) aufge= funden.

Einige Exemplare aus IIb der Gegend Grave: Oss. Wahrscheinlich eine Varietät der vorhergehen= den Art.

Im mittleren Pliocän nicht selten. Wird von LorIÉ aus Ib (Goes) und aus II a (Utrecht) erwähnt.

Diese Form wird von Lorié aus Ib (Goes) er= wähnt. Wahrscheinlich eine Varietät der Capulus ungaricus. Mir aus unsrem Boden nicht bekannt.

Im mittleren Pliocän der Gegend Grave=Oss sehr häufig. Von Lorié aus Ib (Goes) und III a (Utrecht, Amsterdam) erwähnt. Auch von mir in III a (Oude= water, Barendrecht) auf= gefunden.

Im mittleren Pliocän der Gegend Grave=Oss ziem= lich häufig. Auch häufig in III a (Oudewater, Baren= drecht). 
$\mathrm{h}=$,sehr häufig" bis ,nicht selten".

$\mathrm{S}=$,selten" bis "sehr selten".

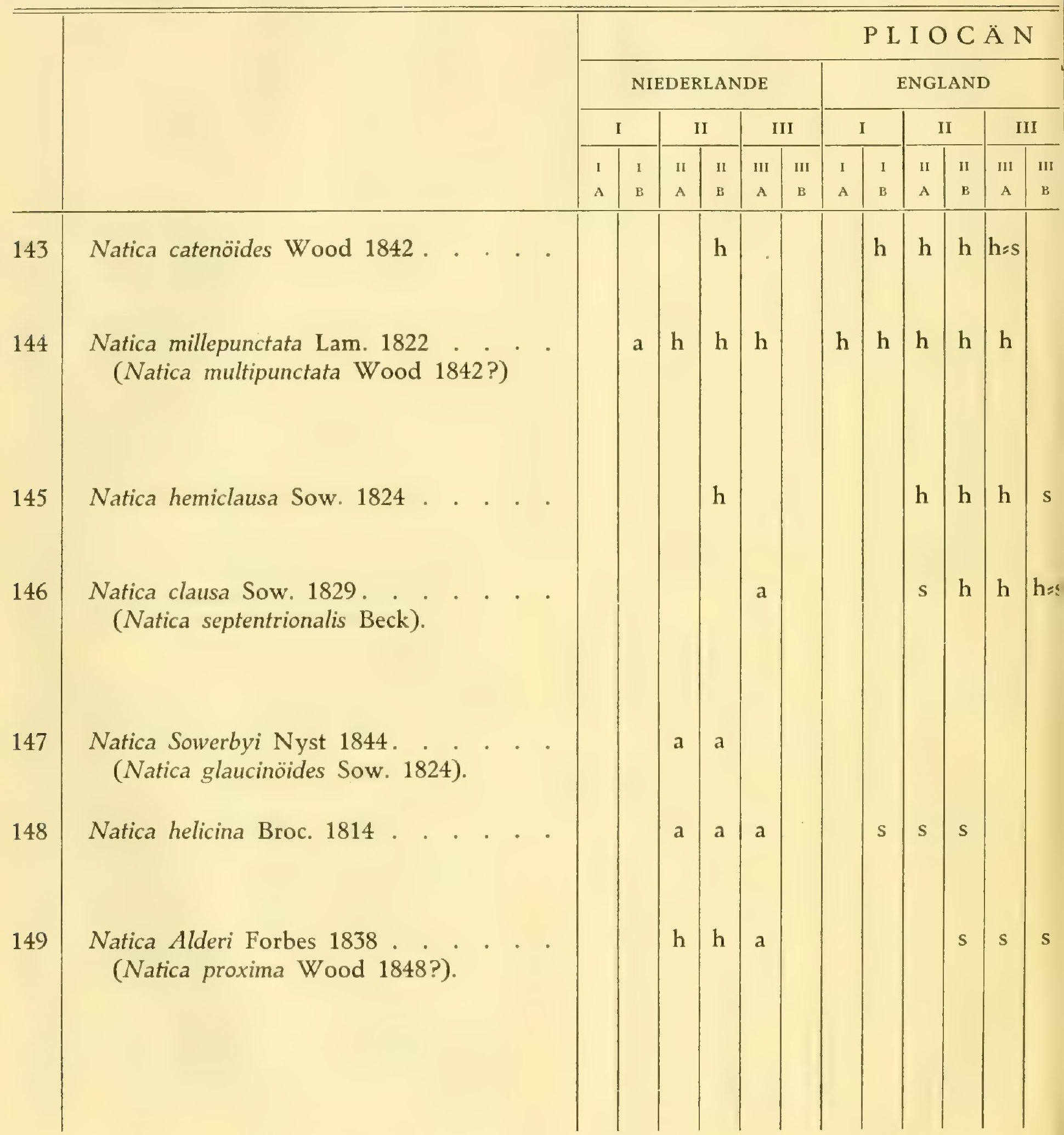




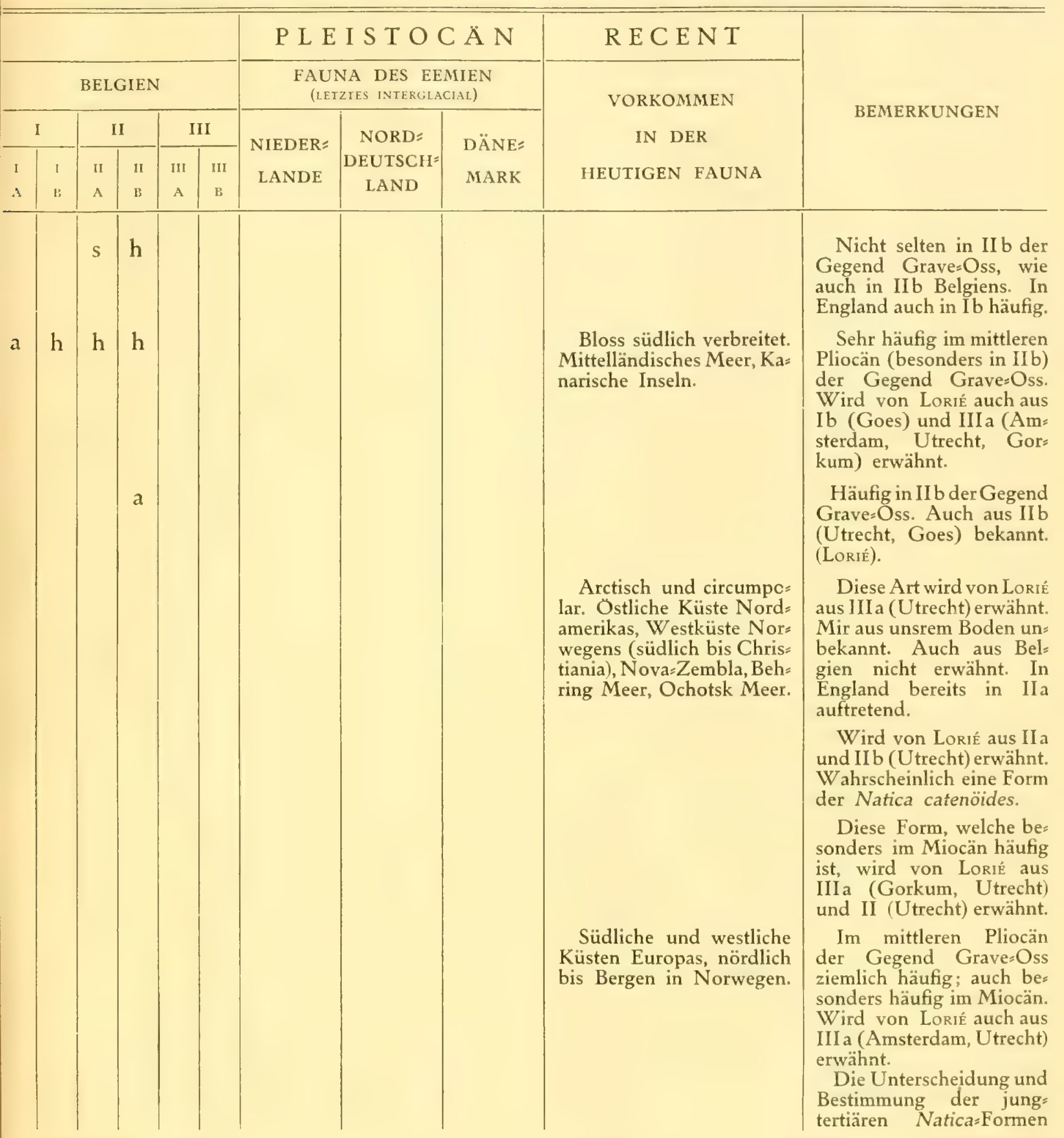


$\mathrm{h}=$ „sehr häufig” bis "nicht selten”.

$\mathrm{S}=$ „selten” bis „sehr selten".

\begin{tabular}{|c|c|c|c|c|c|c|c|c|c|c|c|c|c|c|c|c|}
\hline & & & & & \multicolumn{12}{|c|}{ P L I O C $\ddot{A} N$} \\
\hline & & & & & \multicolumn{6}{|c|}{ NIEDERLANDE } & \multicolumn{6}{|c|}{ ENGLAND } \\
\hline & & & & & & & & & & & & I & & & & II \\
\hline & & & & & $\begin{array}{l}\mathrm{I} \\
\mathrm{A}\end{array}$ & $\begin{array}{l}\text { I } \\
\text { B }\end{array}$ & $\begin{array}{l}\text { II } \\
\text { A }\end{array}$ & $\begin{array}{l}\text { II } \\
\text { B }\end{array}$ & III & $\begin{array}{l}\text { III } \\
\text { B }\end{array}$ & I & $\begin{array}{l}\text { I } \\
\text { B }\end{array}$ & $\begin{array}{l}\text { II } \\
\text { A }\end{array}$ & $\begin{array}{l}\text { II } \\
\text { в }\end{array}$ & III & III \\
\hline 150 & Hydrobia ulvae Penn. 1776. . . & . & . & . & & & & $a$ & a & & & & & & s & $\mathrm{s}$ \\
\hline 151 & Hydrobia similis Drap. 1805 . . & . & . & . & & & & & $\mathrm{a}$ & & & & & & & \\
\hline 152 & Rissoa vitrea Mont. 1803 & . & . & . & & & $\mathrm{h}$ & $\mathrm{h}$ & & & & $\mathrm{h}$ & & & & \\
\hline 153 & Rissoa reticulata Mont. 1803 . . & . & . & . & & & & & a & & & $\mathrm{h}$ & $\mathrm{s}$ & $\mathrm{s}$ & & \\
\hline 154 & Rissoa semicostata Woodw. 1833. & . & . & . & & a & & a & & & & & s & s & $\mathrm{s}$ & $\mathrm{s}$ \\
\hline 155 & Rissoa Zetlandica Mont. 1803 . . & . & . & . & & $\mathrm{a}$ & & & & & & $\mathrm{s}$ & $\mathrm{s}$ & $\mathrm{s}$ & $\mathrm{s}$ & \\
\hline
\end{tabular}


$r=$ auf secundärer Lagerstätte.

$\mathrm{a}=$ anwesend, jedoch unbekannt ob häufig oder selten.

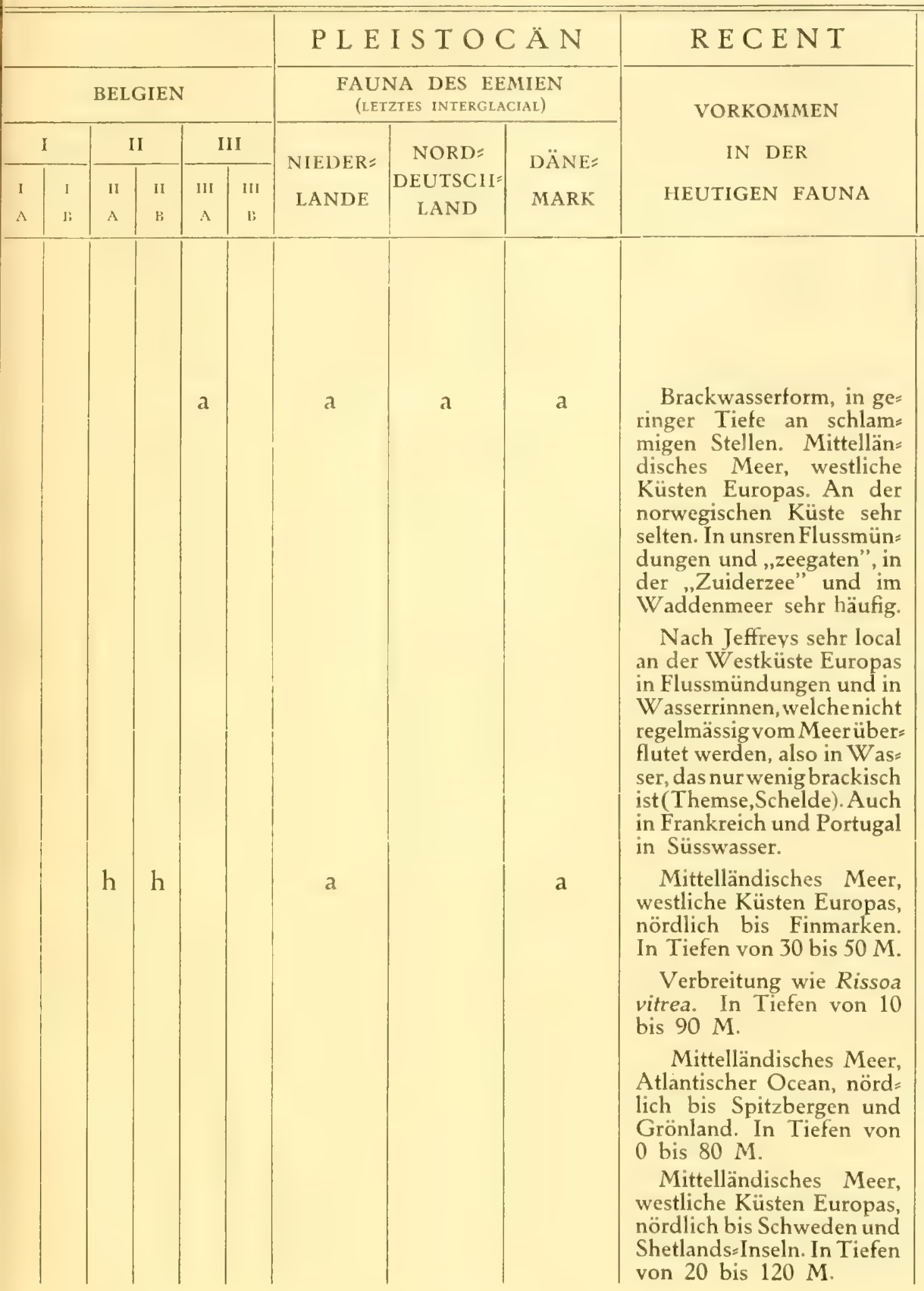

BEMERKUNGEN

scheint mir noch recht mangelhaft: $\mathrm{m}$. E. müssen mehrere "Arten" unter einem Namen vereinigt werden.

Wird von LorIÉ aus II b (Goes) und III a (Amster: dam) erwähnt. Mir aus unsrem Pliocän nicht be kannt.

Wird von LoRIÉ aus III a (Amsterdam) erwähnt. Im englischen und belgischen Pliocän unbekannt.

Ziemlich häufig im mitt= leren Pliocän der Gegend Grave=Oss.

Wird von LORIÉ aus III a (Amsterdam)erwähnt.

Wird von LorIÉ aus Ib (Goes) und IIb (Utrecht) erwähnt.

Wird von LoRIÉ aus Ib (Goes) erwähnt. 
$\mathrm{h}=$ „sehr häufig" bis ,nicht selten".

$\mathrm{s}=$ "selten" bis "sehr selten".

\begin{tabular}{|c|c|c|c|c|c|c|c|c|c|c|c|c|c|c|c|c|c|}
\hline & & & & & & \multicolumn{12}{|c|}{ P L I OC A N } \\
\hline & & & & & & \multicolumn{6}{|c|}{ NIEDERLANDE } & \multicolumn{6}{|c|}{ ENGLAND } \\
\hline & & & & & & \multicolumn{2}{|c|}{ I } & \multicolumn{2}{|c|}{ II } & \multicolumn{2}{|c|}{ III } & \multicolumn{2}{|c|}{ I } & \multicolumn{2}{|c|}{ II } & \multicolumn{2}{|c|}{ III } \\
\hline & & & & & & I & $\begin{array}{l}\text { I } \\
\text { B }\end{array}$ & $\begin{array}{l}\text { II } \\
\text { A }\end{array}$ & $\begin{array}{l}\text { II } \\
\text { B }\end{array}$ & $\begin{array}{l}\text { III } \\
\text { A }\end{array}$ & $\begin{array}{c}\mathrm{MI} \\
\mathrm{B}\end{array}$ & $\begin{array}{l}\text { I } \\
\text { A }\end{array}$ & $\begin{array}{l}\text { I } \\
\text { B }\end{array}$ & $\begin{array}{l}\text { II } \\
\text { A }\end{array}$ & $\begin{array}{l}\text { II } \\
\text { B }\end{array}$ & III & $\begin{array}{c}\text { III } \\
\text { B }\end{array}$ \\
\hline 156 & Scalaria clathratula Turton 1806 & • & & - & . & & & s & & & & s & $\mathrm{h}$ & & & & \\
\hline 157 & Scalaria subulata Sow. 1825. & . & . & - & . & & & h & h & & & & h & $\mathrm{h}$ & $\mathrm{s}$ & s & \\
\hline 158 & Scalaria foliacea Sow. 1825 . . & - & - & - & . & & & h & & & & & $\mathrm{h}$ & s & $s$ & $s$ & \\
\hline 159 & Scalaria frondicula Wood 1842 & - & • & . & . & & & h & $\mathrm{h}$ & & & & $\mathrm{h}$ & 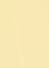 & $\mathrm{s}$ & s & \\
\hline 160 & Scalaria frondosa Sow. 1827 & - & . & . & . & & & s & s & & & & h & . & & & \\
\hline 161 & Scalaria Trevelyana Leach 1820 & - & - & - & . & & & $\mathbf{s}$ & $\mathbf{s}$ & & & & & & $\mathbf{s}$ & $\mathbf{s}$ & s \\
\hline 162 & Scalaria funicula Wood 1874 . & ${ }^{\circ}$ & . & • & . & & & $s$ & & & & & $\mathrm{~h}$ & $s$ & h & $\mathrm{h}$ & \\
\hline
\end{tabular}


h = „sehr häufig" bis ,nicht selten".

$\mathrm{S}=$ „selten” bis „sehr selten".

\begin{tabular}{|c|c|c|c|c|c|c|c|c|c|c|c|c|c|c|c|c|}
\hline & & & & & \multicolumn{12}{|c|}{ P L I OC A N } \\
\hline & & & & & \multicolumn{6}{|c|}{ NIEDERLANDE } & \multicolumn{6}{|c|}{ ENGLAND } \\
\hline & & & & \multicolumn{2}{|c|}{ I } & \multicolumn{2}{|c|}{ II } & \multicolumn{2}{|c|}{ III } & \multicolumn{2}{|c|}{ I } & \multicolumn{2}{|c|}{ II } & \multicolumn{2}{|c|}{ III } \\
\hline & & & & & $\begin{array}{l}\text { I } \\
\text { A }\end{array}$ & $\begin{array}{l}\text { I } \\
\text { B }\end{array}$ & $\begin{array}{l}\text { II } \\
\text { A }\end{array}$ & $\begin{array}{l}\text { II } \\
\text { B }\end{array}$ & $\begin{array}{l}\text { III } \\
\mathrm{A}\end{array}$ & $\begin{array}{c}\text { III } \\
\text { B }\end{array}$ & $\begin{array}{l}\mathrm{I} \\
\mathrm{A}\end{array}$ & $\begin{array}{l}\text { I } \\
\text { B }\end{array}$ & $\begin{array}{l}\text { II } \\
\text { A }\end{array}$ & $\begin{array}{l}\text { II } \\
\text { B }\end{array}$ & $\begin{array}{l}\text { III } \\
\text { A }\end{array}$ & $\begin{array}{c}\text { III } \\
\text { B }\end{array}$ \\
\hline 163 & Turritella incrassata Sow. 1814. & • & - & - & & a & h & h & & & & h & h & $\mathrm{h}$ & $\mathrm{h}$ & h \\
\hline 164 & $\begin{array}{l}\text { Turritella terebra Linn. } 1766 \text {. } \\
\quad \text { (Turritella communis Risso 1826). }\end{array}$ & & • & - & & a? & & $\mathbf{a}$ & h & & & & $\mathbf{S}$ & $\mathbf{s}$ & $\mathrm{s}$ & $\mathrm{h}$ \\
\hline 165 & Turritella subangulata Broc. 1814. & . & . & • & & & $\mathrm{r}$ & & & & & $\mathbf{S}$ & s & s & $\mathbf{s}$ & \\
\hline 166 & Vermetus intortus Lam. 1822 . . & . & . & $\cdot$ & & & s & $\mathbf{a}$ & & & & h & & s & s & \\
\hline 167 & Turbonilla internodula Wood 1847 & . & • & - & & & $\mathbf{s}$ & & & & & $\mathrm{h}$ & h & $\mathrm{h}$ & $\mathrm{h}$ & $S$ \\
\hline 168 & Turbonilla similis Forbes 1838 . . & - & . & · & & & s & s & $\mathrm{a}$ & & & s & $s$ & s & & \\
\hline 169 & Turbonilla filosa Wood 1842 . & • & . & - & & & s & & & & & s & s & s & $s$ & \\
\hline
\end{tabular}




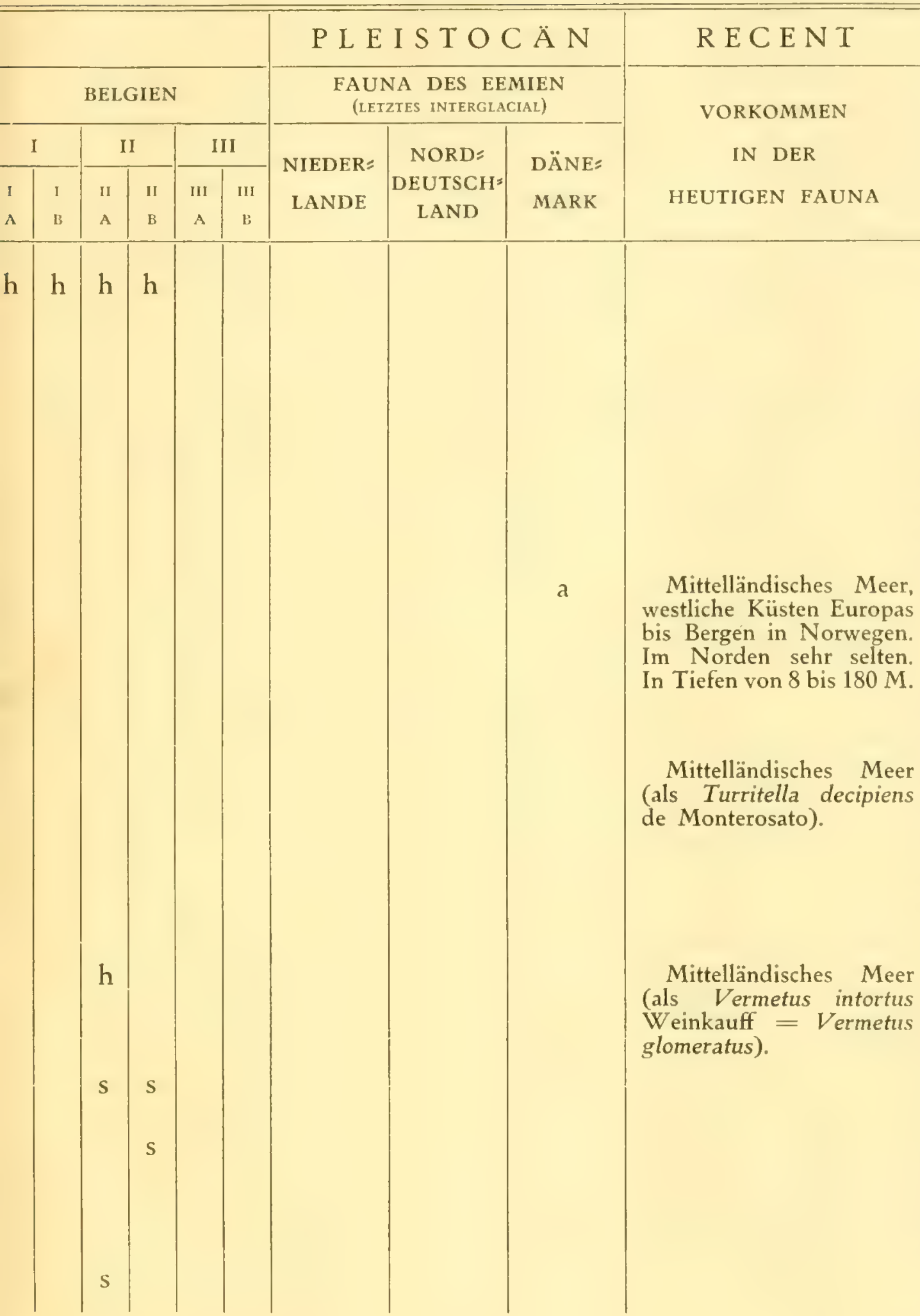

Im mittleren Pliocän überall sehr häufig. Wird von LoRIÉ auch aus Ib (Goes) erwähnt. Diese Art ist sehr variabel und bildet viele Varietäten, welche einerseits sich der recenten Form $T$. terebra nähern, andrerseits auch den miocänen und oligo= cänen Formen sehr ähnlich sind.

Im oberen Pliocän ziem= lich häufig (Amsterdam, Utrecht, Gorkum, Oude= water, Barendrecht, Breda). Auch nach Lorié aus IIb (Utrecht) und Ib (Goes) bekannt.

Diese bekannte miocäne Form findet sich im un= teren II a der Gegend Grave=Oss oft auf secun= därer Lagerstätte. Im eng= lischen Pliocän sehr wahr: scheinlich ebenfalls aus dem Miocän stammend.

In II a der Gegend Grave $=$ Oss ziemlich selten. Wird von LORIÉ auch aus II b (Utrecht) genannt.

Ziemlich selten in II a der Gegend Grave=Oss.

Ziemlich selten im mitt= leren Pliocän der Gegend Grave=Oss. Wird vonLorIÉ auch aus III a (Amsterdam) erwähnt.

In IIa der Gegend Grave=Oss sehr selten. 
h = „sehr häufig" bis ,nicht selten".

$\mathrm{s}=$,selten" bis "sehr selten”.

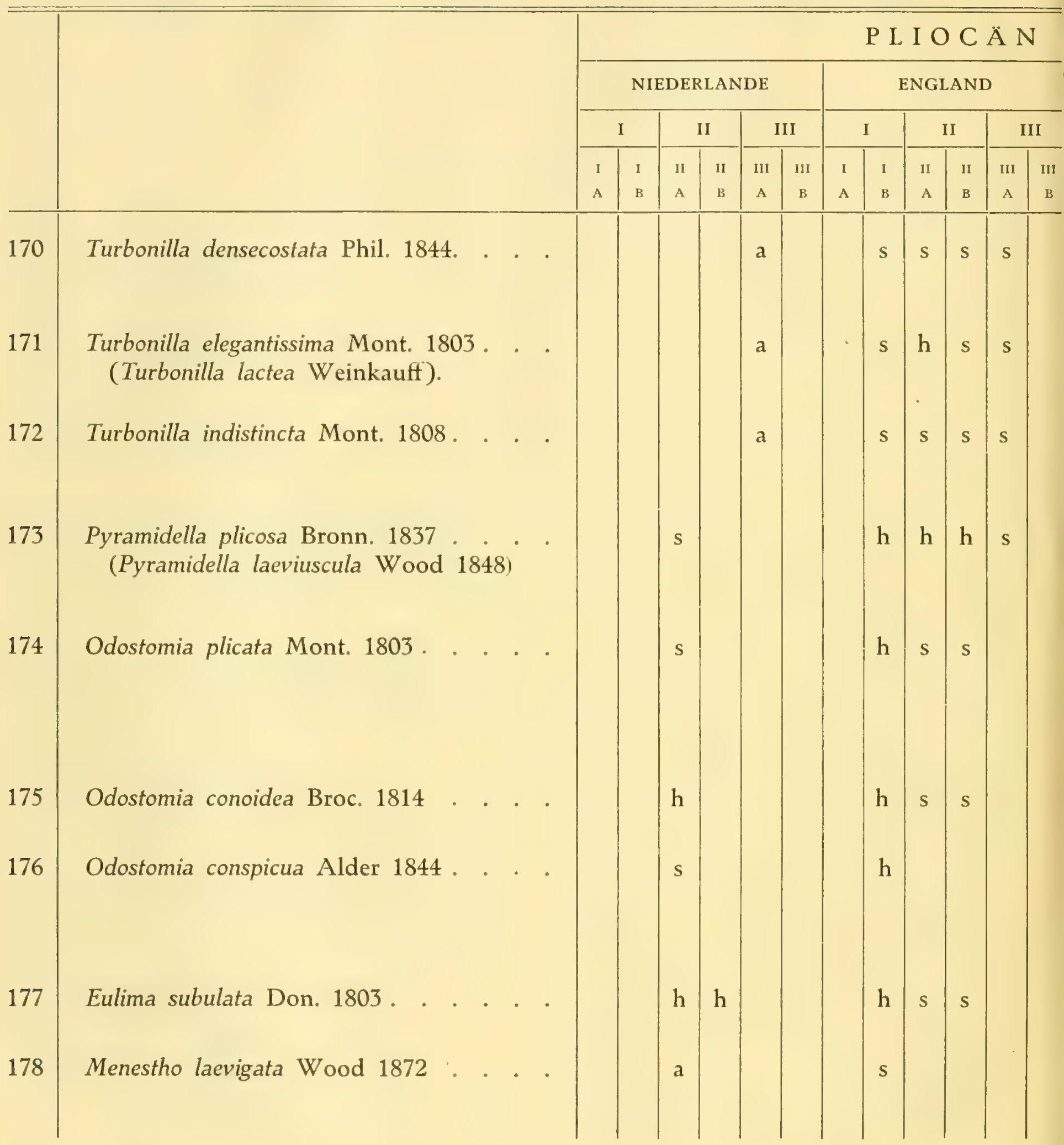


$\mathrm{r}=$ auf secundärer Lagerstätte.

$\mathbf{a}=$ anwesend, jedoch unbekannt ob häufig oder selten.

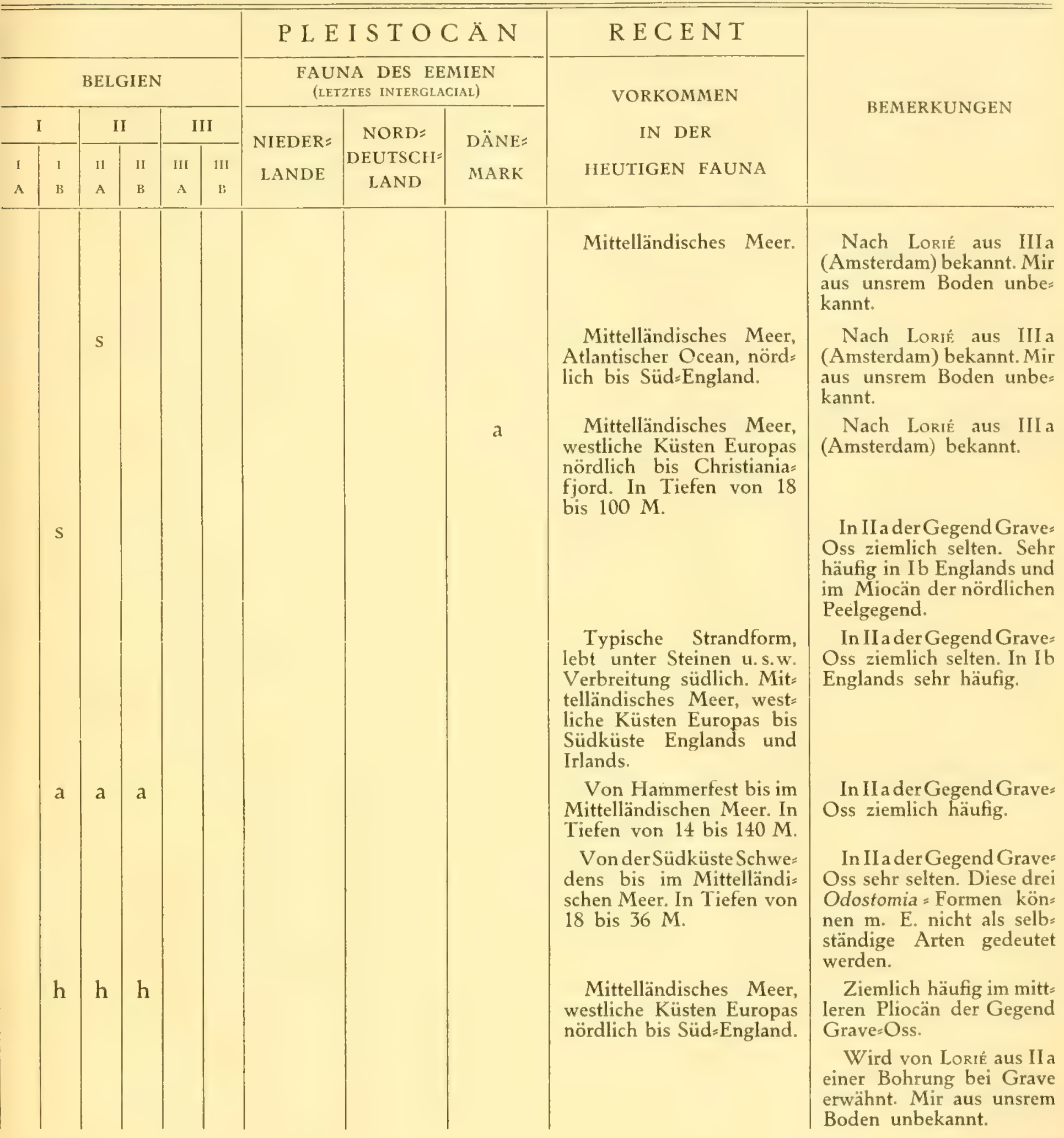


$\mathrm{h}=$ „sehr häufig" bis „,nicht selten”.

$\mathrm{s}=$,selten" bis „sehr selten".

\begin{tabular}{|c|c|c|c|c|c|c|c|c|c|c|c|c|c|c|c|c|}
\hline & & & & & \multicolumn{12}{|c|}{$\mathrm{PLI} O \mathrm{C} \ddot{\mathrm{N}}$} \\
\hline & & & & & \multicolumn{6}{|c|}{ NIEDERLANDE } & \multicolumn{6}{|c|}{ ENGL.AND } \\
\hline & & & & & & & & I & & II & & & & & & II \\
\hline & & & & & ${ }_{\mathrm{A}}^{\mathrm{I}}$ & $\begin{array}{l}\text { I } \\
\text { B }\end{array}$ & $\begin{array}{l}\text { II } \\
\text { A }\end{array}$ & $\begin{array}{l}\text { II } \\
\text { B }\end{array}$ & III & $\begin{array}{c}\text { III } \\
\text { в }\end{array}$ & $\begin{array}{l}\mathrm{I} \\
\mathrm{A}\end{array}$ & $\begin{array}{l}\text { I } \\
\text { B }\end{array}$ & $\begin{array}{l}\text { II } \\
\text { A }\end{array}$ & $\begin{array}{l}\text { } 11 \\
\text { B }\end{array}$ & III & BI \\
\hline 179 & Cerithium tricinctum Broc. 1816 & & . & $\bullet$ & & & & $\mathrm{h}$ & a & & $\mathrm{h}$ & s & h & h & h & \\
\hline 180 & Cerithium punctulum Nyst 1881 & & • & . & & & $s$ & & & & & & & s & & \\
\hline 181 & Cerithium tuberculare Mont. 1803 & & · & . & & a & & & $\mathbf{a}$ & & & $\mathrm{h}$ & s & $s$ & $s$ & \\
\hline 182 & Aporrhais pes=pelecani Linn. 1766 & & . & . & & & h & $\mathrm{h}$ & & & $\mathrm{h}$ & $\mathrm{h}$ & $\mathrm{h}$ & h & $h=s$ & \\
\hline 183 & Cypraea europaea Mont. 1803. & & $\theta^{\prime}$ & . & & & s & & & & s & h & $\mathrm{h}$ & $\mathrm{h}$ & $\mathrm{h}$ & \\
\hline 184 & Cassis saburon Brug. 1792 . . & & & & & & $\mathrm{r}$ & & & & & & & & r & \\
\hline 185 & Cassidaria bicatenata Sow. 1824 & & . & s. & & & & a & $a$ & & & s & s & s & $\mathrm{h}$ & \\
\hline
\end{tabular}


h = „sehr häufig” bis ,nicht selten”.

$\mathrm{s}=$,selten" bis "sehr selten".

\begin{tabular}{|c|c|c|c|c|c|c|c|c|c|c|c|c|c|c|c|c|c|c|}
\hline & & & & & & & \multicolumn{12}{|c|}{$\mathrm{P} L \mathrm{I} O \mathrm{O} \ddot{\mathrm{N}}$} \\
\hline & & & & & & & \multicolumn{6}{|c|}{ NIEDERLANDE } & \multicolumn{6}{|c|}{ ENGLAND } \\
\hline & & & & & & & 1 & & I & & & & & & I & & II & \\
\hline & & & & & & & $\begin{array}{l}1 \\
\mathrm{~A}\end{array}$ & $\begin{array}{l}\mathrm{I} \\
\mathrm{E}\end{array}$ & $\begin{array}{l}\text { II } \\
\text { A }\end{array}$ & $\begin{array}{l}\text { II } \\
\text { E }\end{array}$ & $\begin{array}{c}\text { III } \\
\text { A }\end{array}$ & $\begin{array}{c}\text { III } \\
\mathbf{E}\end{array}$ & $\begin{array}{l}\text { I } \\
\text { A }\end{array}$ & $\begin{array}{l}\text { I } \\
\text { B }\end{array}$ & II & $\begin{array}{l}\mathrm{II} \\
\mathrm{B}\end{array}$ & $\begin{array}{l}\text { III } \\
\text { A }\end{array}$ & $\begin{array}{l}\text { III } \\
\text { E }\end{array}$ \\
\hline 186 & Ficula reticulata Lam. 1835 . . & & & & 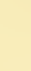 & . & & & r & & & & $\mathrm{h}$ & s & & & $\mathbf{r}$ & \\
\hline 187 & Columbella subulata Broc. 1824 & & & & 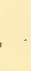 & $\cdot$ & & & $\mathrm{h}$ & & & & & & & 's & & \\
\hline 188 & Columbella sulcata Sow. 1823 . & & & & 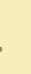 & $\cdot$ & & & s & & & & & $\mathrm{s}$ & $\mathrm{h}$ & h & $h=s$ & \\
\hline 189 & Buccinum undatum Linn. 1766. & & & & . & . & & & $\mathrm{s}$ & $\mathbf{s}$ & $a$ & & & h & $\mathrm{h}$ & $\mathrm{h}$ & h & $\mathrm{h}$ \\
\hline 190 & Buccinopsis Dalei Sow. 1825 & & & & 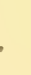 & . & & & s & s & & & $\mathrm{s}$ & $\mathrm{h}$ & $\mathrm{h}$ & $\mathrm{h}$ & $\mathrm{h}$ & s \\
\hline 191 & Nassa reticosa Sow. 1815 & & & & v & . & & & $\mathrm{h}$ & h & a & & & $\mathrm{h}$ & $\mathrm{h}$ & $\mathrm{h}$ & h & s \\
\hline 192 & Nassa serrata Broc. 1814 & & & & • & . & & & & $h^{\circ}$ & & & & & & $s$ & & \\
\hline
\end{tabular}




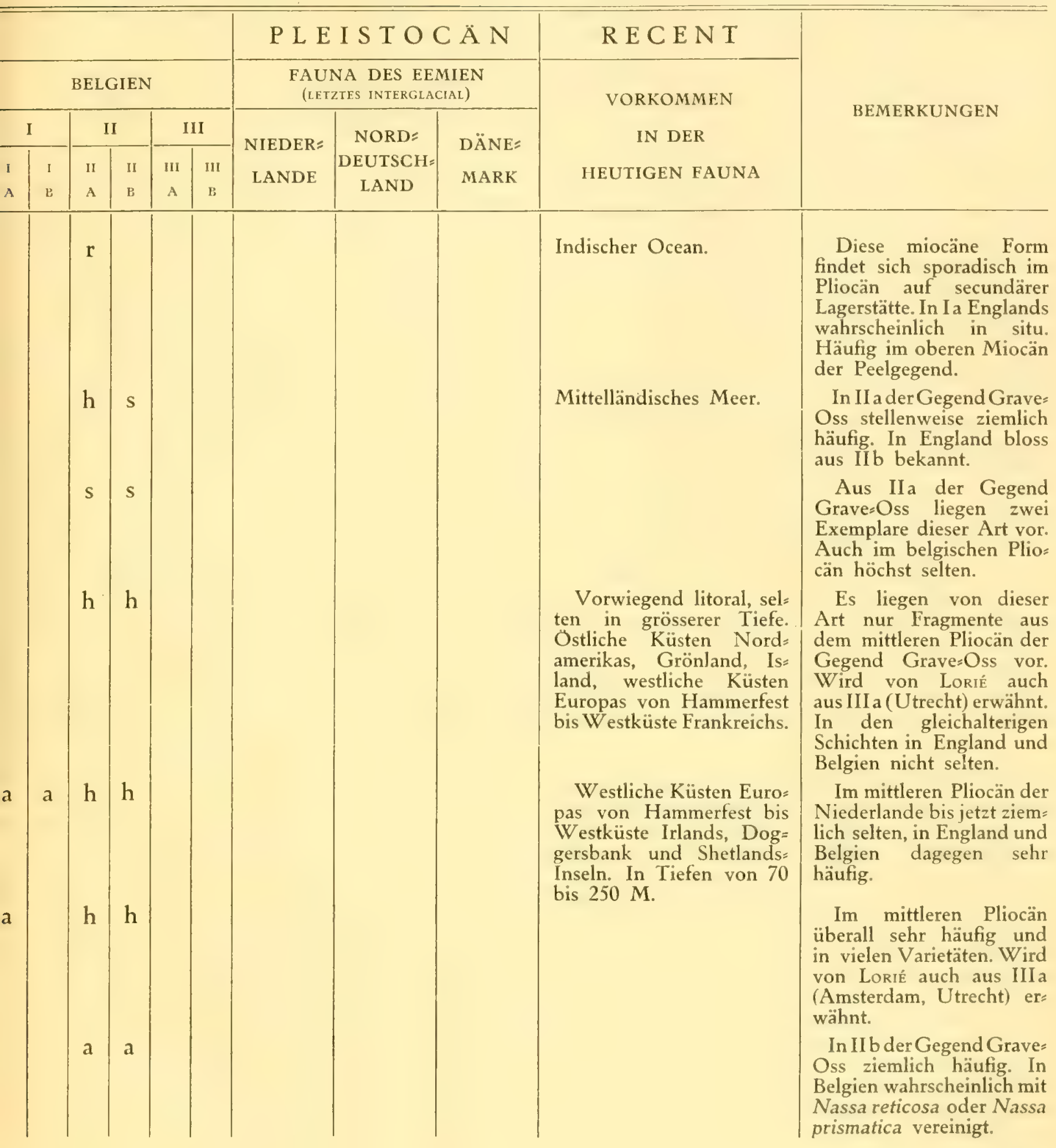


$\mathrm{h}=$ „sehr häufig" bis ,nicht selten".

$\mathrm{s}=$,selten” bis „sehr selten”.

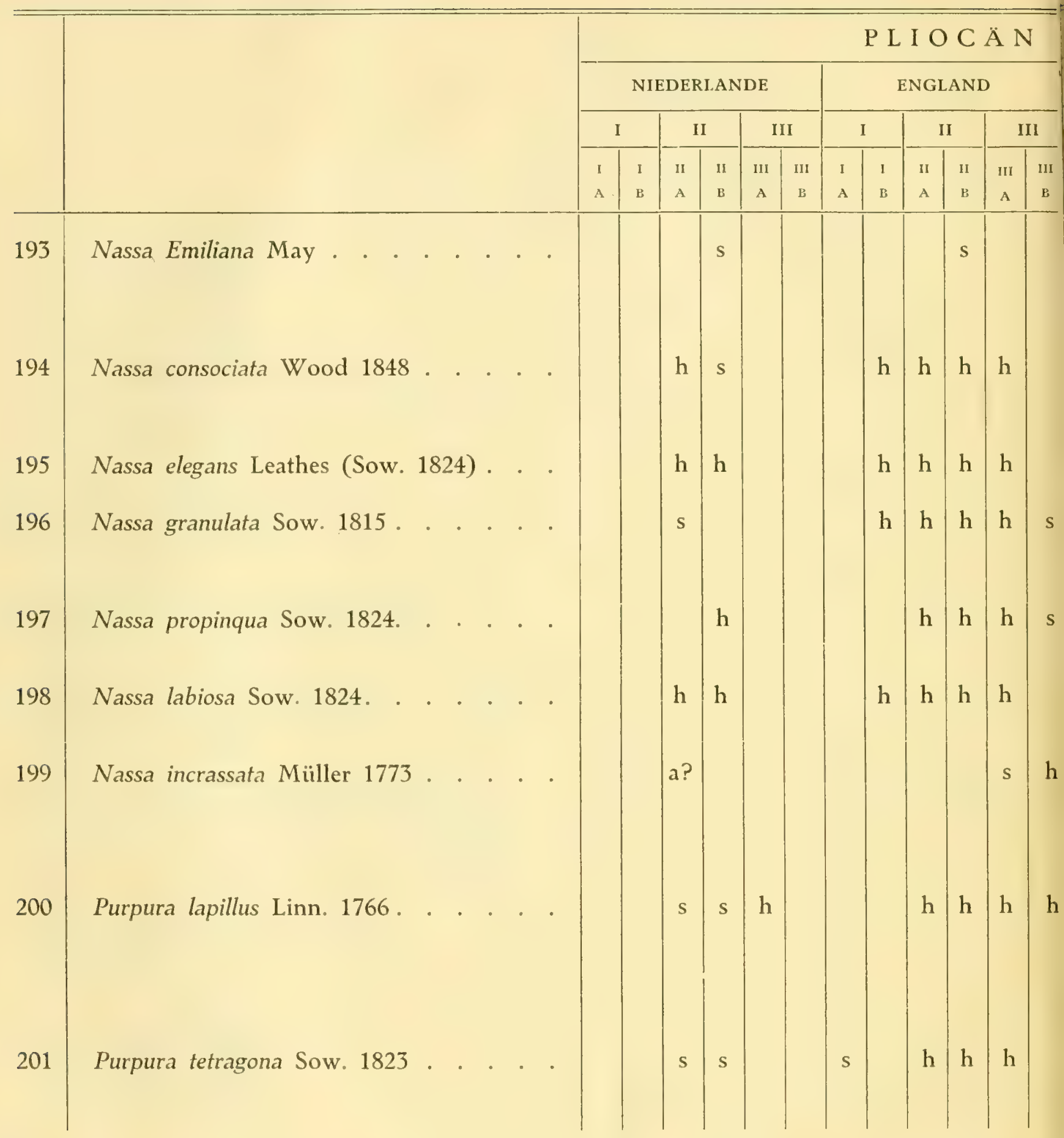


$\mathrm{r}=$ auf secundärer Lagerstätte.

$a=$ anwesend, jedoch unbekannt ob häufig oder selten.

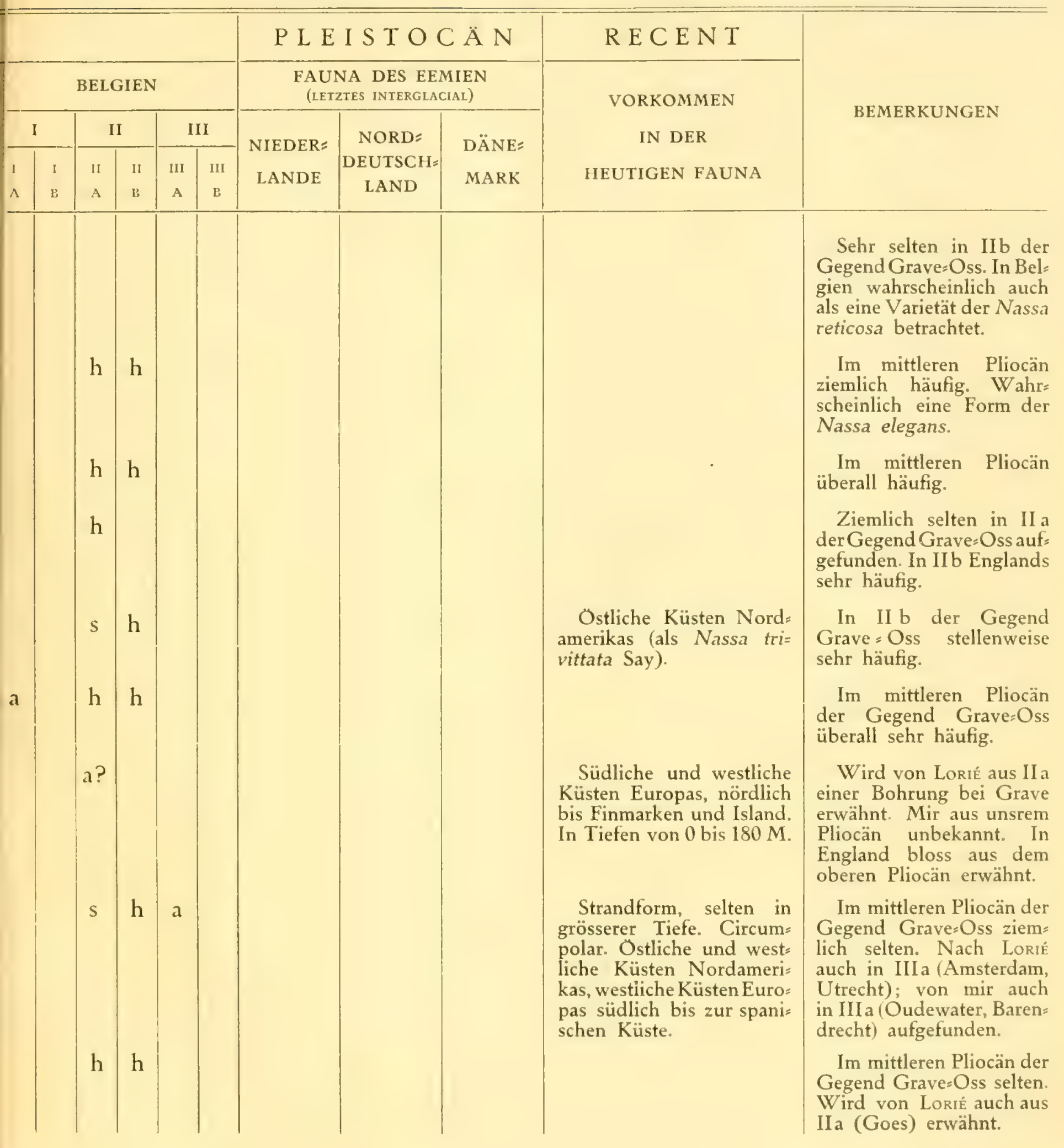


h := "sehr häufig" bis ,nicht selten".

$\mathrm{S}=$,selten" bis "sehr selten”.

\begin{tabular}{|c|c|c|c|c|c|c|c|c|c|c|c|c|c|c|c|c|}
\hline & & & & & \multicolumn{12}{|c|}{ P L I O C $\AA$ N } \\
\hline & & & & & \multicolumn{6}{|c|}{ NIEDERLANDE } & \multicolumn{6}{|c|}{ ENGLAND } \\
\hline & & & & & \multicolumn{2}{|c|}{ I } & \multicolumn{2}{|c|}{ II } & \multicolumn{2}{|c|}{ III } & \multicolumn{2}{|c|}{ I } & \multicolumn{2}{|c|}{ II } & \multicolumn{2}{|c|}{ III } \\
\hline & & & & & 1 & $\begin{array}{l}\text { I } \\
\text { B }\end{array}$ & $\begin{array}{l}\text { и } \\
\text { A }\end{array}$ & $\begin{array}{l}\text { II } \\
\text { B }\end{array}$ & ${ }_{\mathrm{A}}^{\mathrm{III}}$ & ${ }_{\mathrm{B}}^{\mathrm{III}}$ & $\mathrm{I}$ & $\begin{array}{l}\text { I } \\
\text { B }\end{array}$ & $\begin{array}{l}\mathrm{II} \\
\mathrm{A}\end{array}$ & $\begin{array}{l}\text { II } \\
\text { B }\end{array}$ & $\begin{array}{l}\text { III } \\
\text { A }\end{array}$ & $\begin{array}{l}\text { III } \\
\text { E }\end{array}$ \\
\hline 202 & $\begin{array}{l}\text { Trophon alveolatum Sow. } 1826 . \\
\text { (Murex alveolatus Nyst } 1881 \\
\text { Fusus alveolatus Sow. 1826) }\end{array}$ & $\cdot$ & . & . & & a & s & s & & & & $\mathrm{h}$ & $s$ & s & $s$ & \\
\hline 203 & Trophon consociale Wood 1848 . & . & . & . & & & & & a & & & $\mathrm{h}$ & s & s & $s$ & \\
\hline 204 & $\begin{array}{l}\text { Trophon muricatum Mont. } 1803 \\
\quad \text { (Murex muricatus Mont. 1803) }\end{array}$ & . & . & . & & & & h & & & s & h & $\mathrm{h}$ & $\mathrm{h}$ & $\mathrm{h}$ & \\
\hline 205 & Trophon costiferum Wood 1848 . & . & ${ }^{\circ}$ & . & & & & & a? & & & $\mathrm{h}$ & h & $\mathrm{h}$ & $\mathrm{h}$ & \\
\hline 206 & $\begin{array}{l}\text { Trophon scalariforme Gould } 1841 \\
\text { (Tritonium clathratum Lovén } 1846\end{array}$ & & . & . & & & & & a & & & & $s$ & $\mathrm{~h}$ & $\mathrm{~h}$ & s \\
\hline 207 & Typhis fistulosus Broc. 1814 & • & • & . & & & $\mathrm{r}$ & & & & & & & & & \\
\hline
\end{tabular}


$r=$ auf secundärer Lagerstätte.

$a=$ anwesend, jedoch unbekannt ob häufig oder selten.

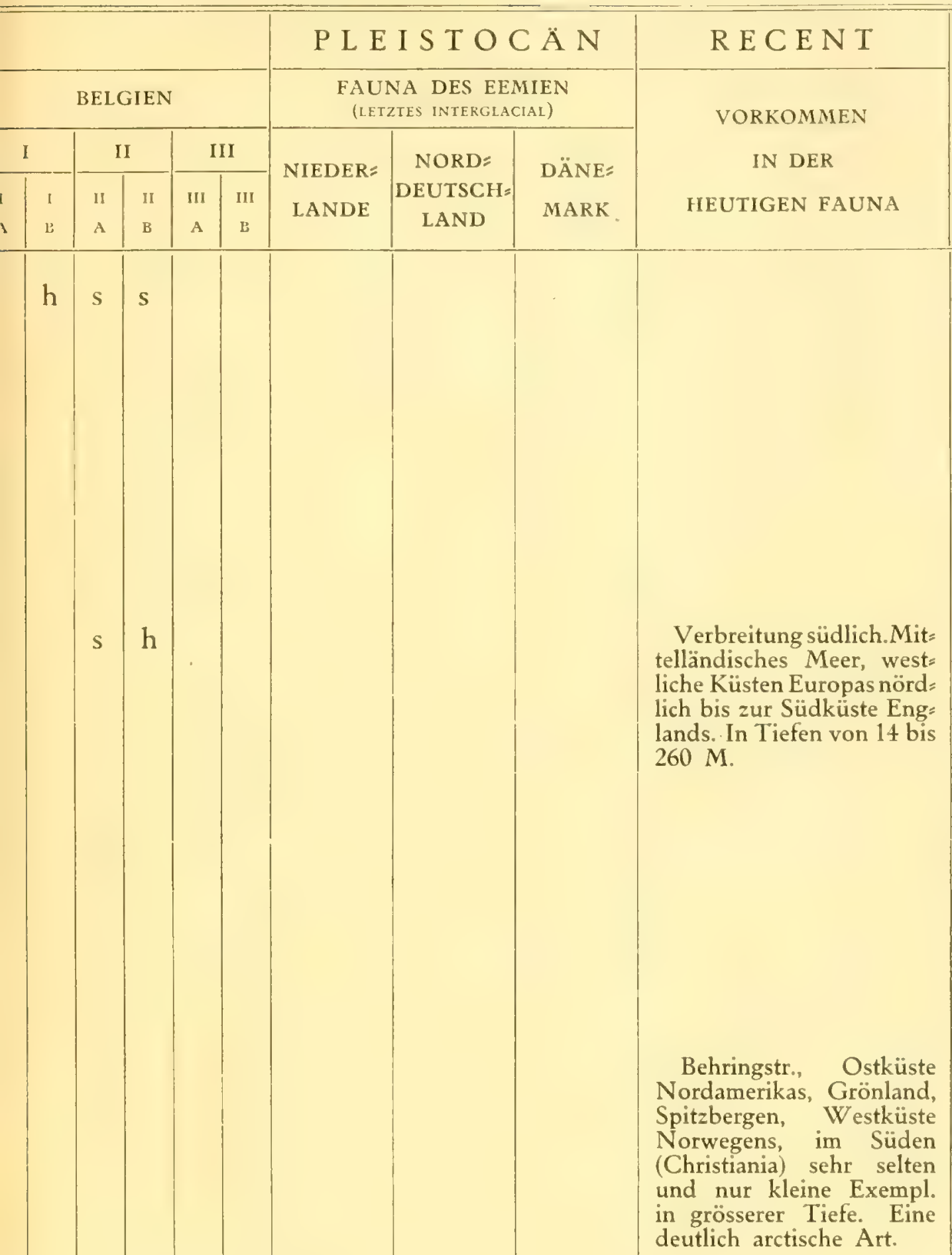

Ein Exemplar, welches vielleicht $z u$ dieser Art gehört, wird von LORIÉ aus III a (Amsterdam) er= wähnt. In England beson= ders im mittleren Pliocän sehr häufig; fehlt dagegen gänzlich in den gleichalte= rigen Schichten der Nieder= lande und Belgiens.

Wird von Lorié aus III a (Amsterdam) erwähnt. Wie die vorhergehende Art im belgischen Pliocän gänzlich unbekannt.

Diese miocäne Art fin= det sich sporadisch in IIa der Gegend Grave=Oss auf secundärer Lagerstätte. 
h:= „sehr häufig” bis ,nicht selten".

$\mathrm{s}=$,selten" bis "sehr selten".

\begin{tabular}{|c|c|c|c|c|c|c|c|c|c|c|c|c|c|}
\hline & & \multicolumn{12}{|c|}{ P L I OC $\ddot{A} \mathrm{~N}$} \\
\hline & & \multicolumn{6}{|c|}{ NIEDERLANDE } & \multicolumn{6}{|c|}{ ENGLAND } \\
\hline & & \multicolumn{2}{|c|}{ I } & \multicolumn{2}{|c|}{ II } & \multicolumn{2}{|c|}{ III } & \multicolumn{2}{|c|}{ I } & \multicolumn{2}{|c|}{ II } & \multicolumn{2}{|c|}{ III } \\
\hline & & $\begin{array}{l}\mathrm{I} \\
\mathrm{A}\end{array}$ & $\begin{array}{l}\text { I } \\
\text { B }\end{array}$ & $\begin{array}{l}\text { II } \\
\text { A }\end{array}$ & $\begin{array}{l}\text { II } \\
\text { B }\end{array}$ & $\begin{array}{c}111 \\
\mathrm{~A}\end{array}$ & $\begin{array}{c}\mathrm{II} \\
\mathrm{B}\end{array}$ & $\begin{array}{l}\mathrm{I} \\
\mathrm{A}\end{array}$ & $\begin{array}{l}\text { I } \\
\text { B }\end{array}$ & $\begin{array}{l}\text { II } \\
\text { A }\end{array}$ & $\begin{array}{l}\text { II } \\
\text { B }\end{array}$ & $\begin{array}{c}\text { III } \\
\text { A }\end{array}$ & $\begin{array}{l}\text { III } \\
\text { B }\end{array}$ \\
\hline 208 & $\begin{array}{l}\text { Fusus gracilis da Costa 1778 . . . . . } \\
\quad \text { (Sipho curta Jeffreys 1847). }\end{array}$ & & & & $\mathrm{h}$ & a & & & & & h & $\mathrm{h}$ & \\
\hline 209 & Fusus elegans Charlesw. 1837 . . . . . & & & & & a & & & s & & $s$ & s & \\
\hline 210 & $\begin{array}{l}\text { Neptunea contraria Linn. } 1766 \text { (Müller 1773) } \\
\text { (Chrysodomus contrarius Linn. } \\
\text { Fusus contrarius Linn. } \\
\text { Trophon contrarium Linn.) }\end{array}$ & & & $\mathrm{h}$ & $\mathrm{h}$ & & & & & $\mathrm{h}$ & h & h & $s$ \\
\hline 211 & $\begin{array}{l}\text { Neptunea antiqua Linn. } 1766 \text { (Müller 1777) } \\
\text { (Chrysodomus antiquus Linn. } \\
\text { Fusus antiquus Linn. } \\
\text { Trophon antiquum Linn.) }\end{array}$ & & & & $a$ & & & & & & s & h & $h=$ \\
\hline 212 & Voluta Lamberti Sow. 1816. . . . . . & & & $\mathrm{h}$ & $\mathrm{h}$ & & & & $\mathrm{h}$ & $\mathrm{h}$ & $\mathrm{h}$ & $h=s$ & $\mathbf{s}$ \\
\hline
\end{tabular}


$r=$ auf secundärer Lagerstätte.

$\mathrm{a}=$ anwesend, jedoch unbekannt ob häufig oder selten.

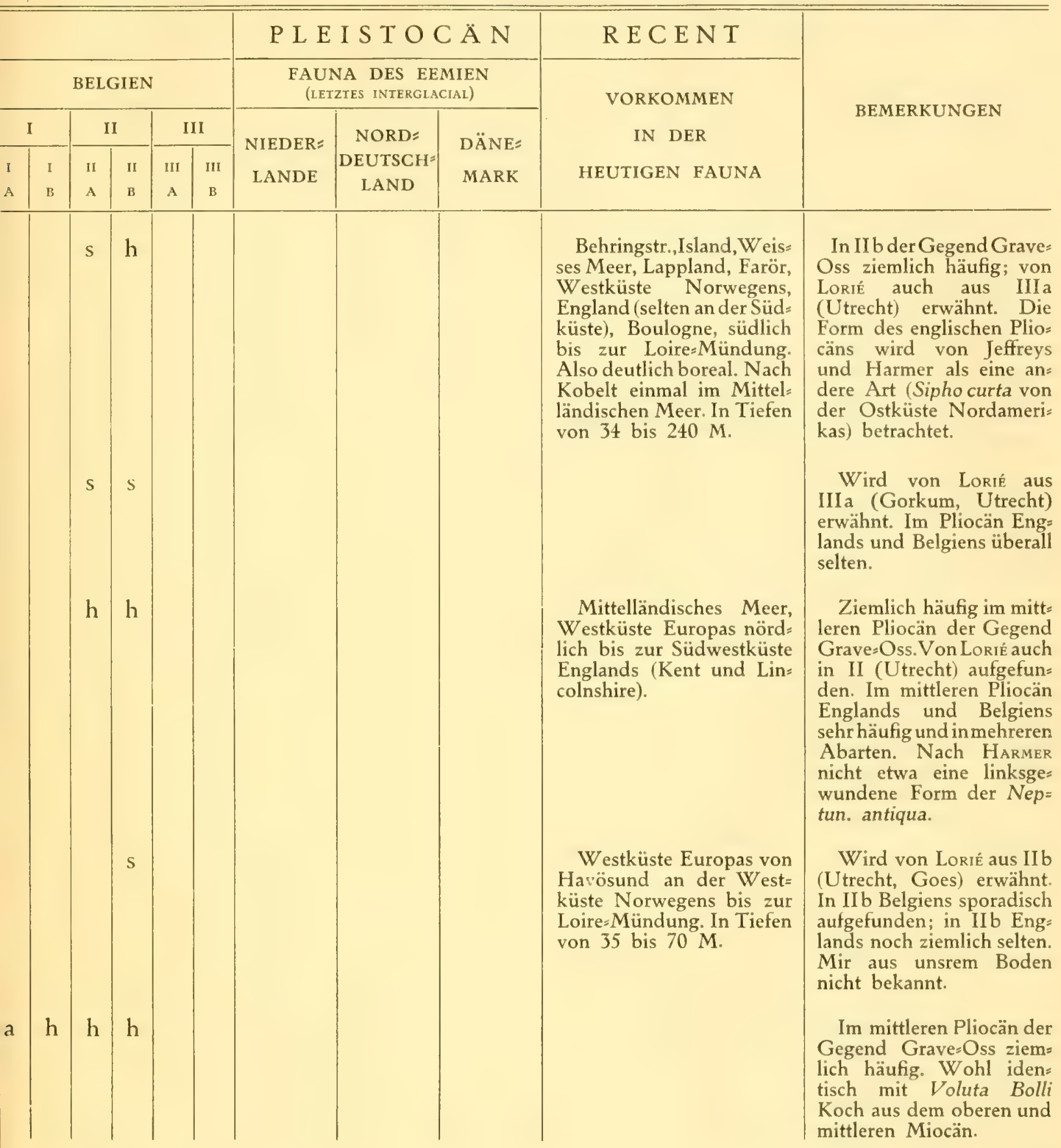


$\mathrm{h}=$ „sehr häufig" bis ,nicht selten".

s = „selten" bis "sehr selten".

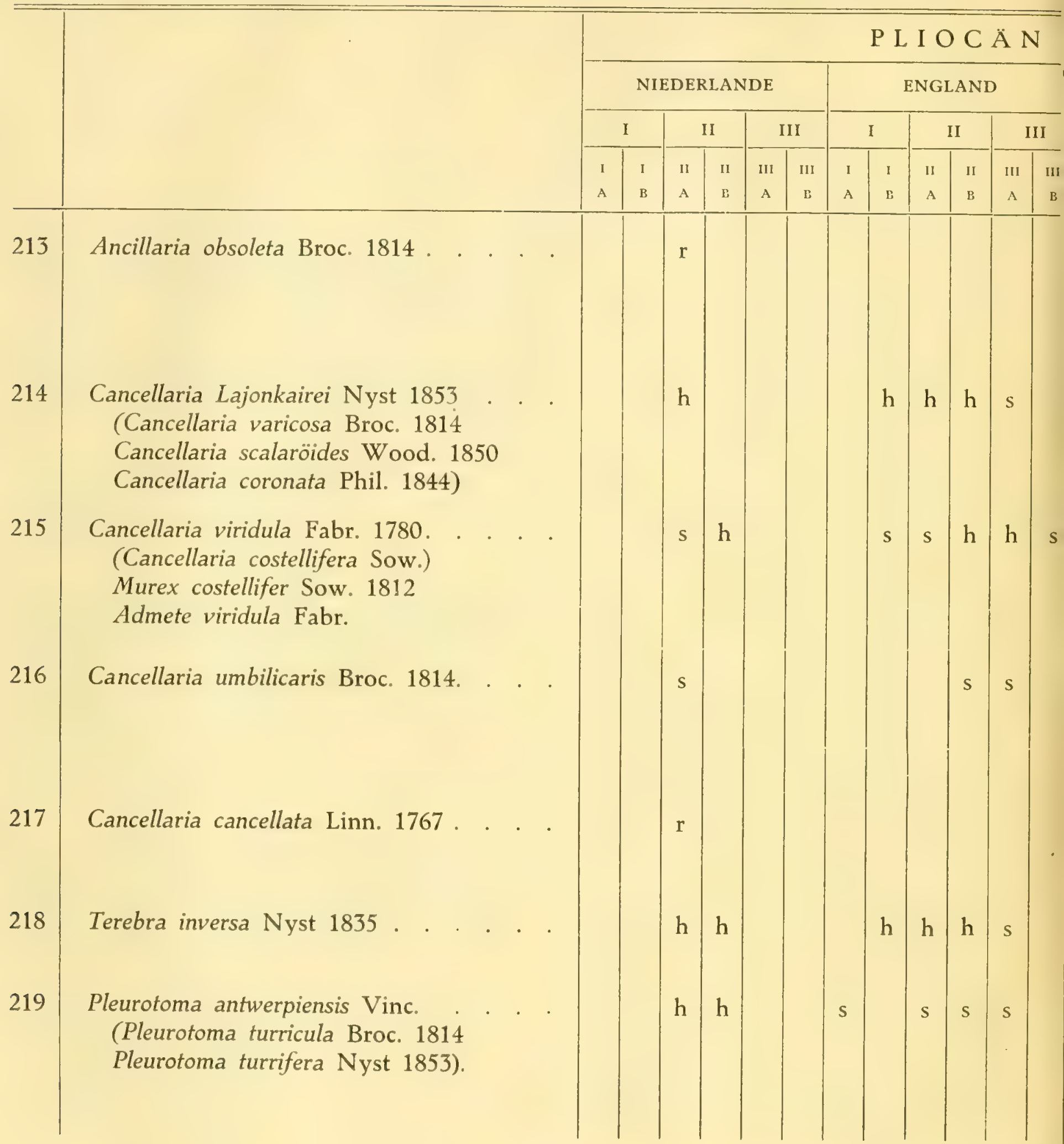


$\mathrm{h}=$ „sehr häufig" bis "nicht selten".

$\mathrm{S}=$,selten” bis „sehr selten”.

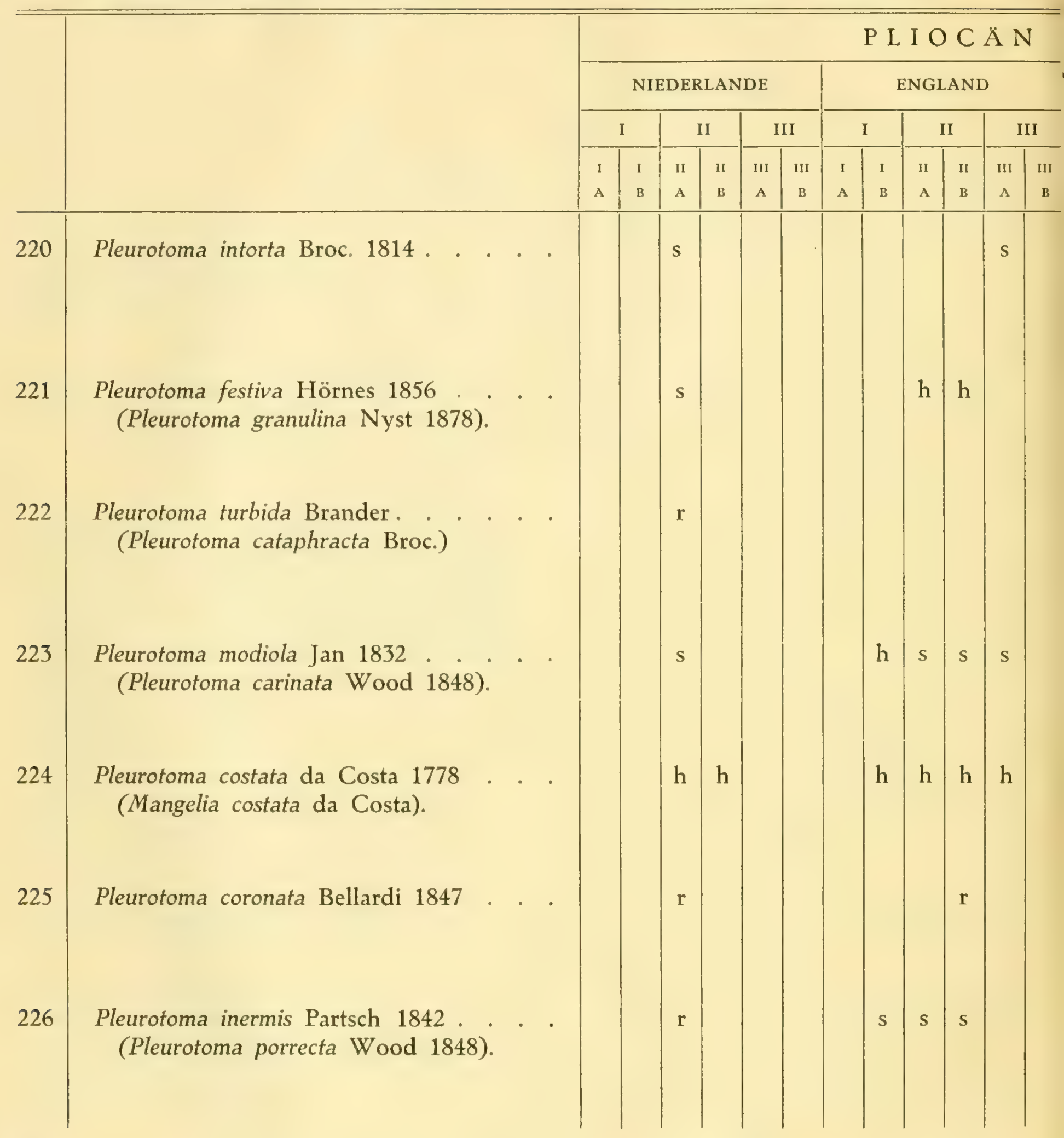


h= „sehr häufig" bis ,nicht selten".

$\mathrm{S}=$ "selten" bis "sehr selten".

\begin{tabular}{|c|c|c|c|c|c|c|c|c|c|c|c|c|c|c|c|}
\hline & & & & \multicolumn{12}{|c|}{ P L I OC $\ddot{A} \mathrm{~N}$} \\
\hline & & & & \multicolumn{6}{|c|}{ NIEDERLANDE } & \multicolumn{6}{|c|}{ ENGLAND } \\
\hline & & & & \multicolumn{2}{|c|}{ I } & \multicolumn{2}{|c|}{ II } & \multicolumn{2}{|c|}{ III } & \multicolumn{2}{|c|}{ I } & \multicolumn{2}{|c|}{ II } & \multicolumn{2}{|c|}{ III } \\
\hline & & & & $\begin{array}{l}\text { I } \\
\text { A }\end{array}$ & $\begin{array}{l}\text { I } \\
\text { B }\end{array}$ & $\begin{array}{l}\text { II } \\
\text { A }\end{array}$ & $\begin{array}{l}\text { II } \\
\text { B }\end{array}$ & $\begin{array}{l}\text { III } \\
\text { A }\end{array}$ & $\begin{array}{l}\text { III } \\
\text { B }\end{array}$ & $\begin{array}{l}\text { I } \\
\text { A }\end{array}$ & $\begin{array}{l}\text { I } \\
\text { B }\end{array}$ & $\begin{array}{l}\text { II } \\
\text { A }\end{array}$ & $\begin{array}{l}\text { II } \\
\text { B }\end{array}$ & $\begin{array}{l}\text { III } \\
\text { A }\end{array}$ & $\begin{array}{c}\text { III } \\
\text { B }\end{array}$ \\
\hline 227 & Pleurotoma perpulchra Wood 1848 . & , & & & & $\mathrm{h}$ & & & & & h & s & & & \\
\hline 228 & $\begin{array}{l}\text { Pleurotoma gracilis Mont. } 1808 \\
\quad \text { (Pleurotoma emarginata Don. } 1803\end{array}$ & & & & & $\mathrm{~s}$ & & & & & & & & & \\
\hline 229 & Pleurotoma brachystoma Phil. 1844 . & • & • & & & s & & a & & & h & h & s & $s$ & \\
\hline 230 & Pleurotoma similis Nyst 1878 . . & • & . & & & h & & & & & & & $\mathbf{s}$ & & \\
\hline 231 & $\begin{array}{l}\text { Pleurotoma Leufroyi Mich. } 1827 \\
\text { (Defrancia Leufroyi Michaud) }\end{array}$ & - : & $\cdot$ & & & & $\mathbf{S}$ & & & & & s & s & s & \\
\hline 232 & Pleurotoma hystrix de Christ et Jan & 18 & 32. & & & s & & & & & s & s & s & & \\
\hline 233 & $\begin{array}{l}\text { Pleurotoma attenuata Weinkauff } \\
\text { var. volvula Bell } \\
\text { (Pleurotoma gracilis Scacchi). }\end{array}$ & . & • & & & S & & & & & $\mathbf{s}$ & & S & & \\
\hline 234 & $\begin{array}{l}\text { Pleurotoma turricula Mont. } 1803 \text {. } \\
\text { (Bela turricula Mont.) }\end{array}$ & $\cdot$ & . & & & & & a & & & & & & h & $h=s$ \\
\hline
\end{tabular}


$r=$ auf secundärer Lagerstätte.

$a=$ anwesend, jedoch unbekannt ob häufig oder selten.

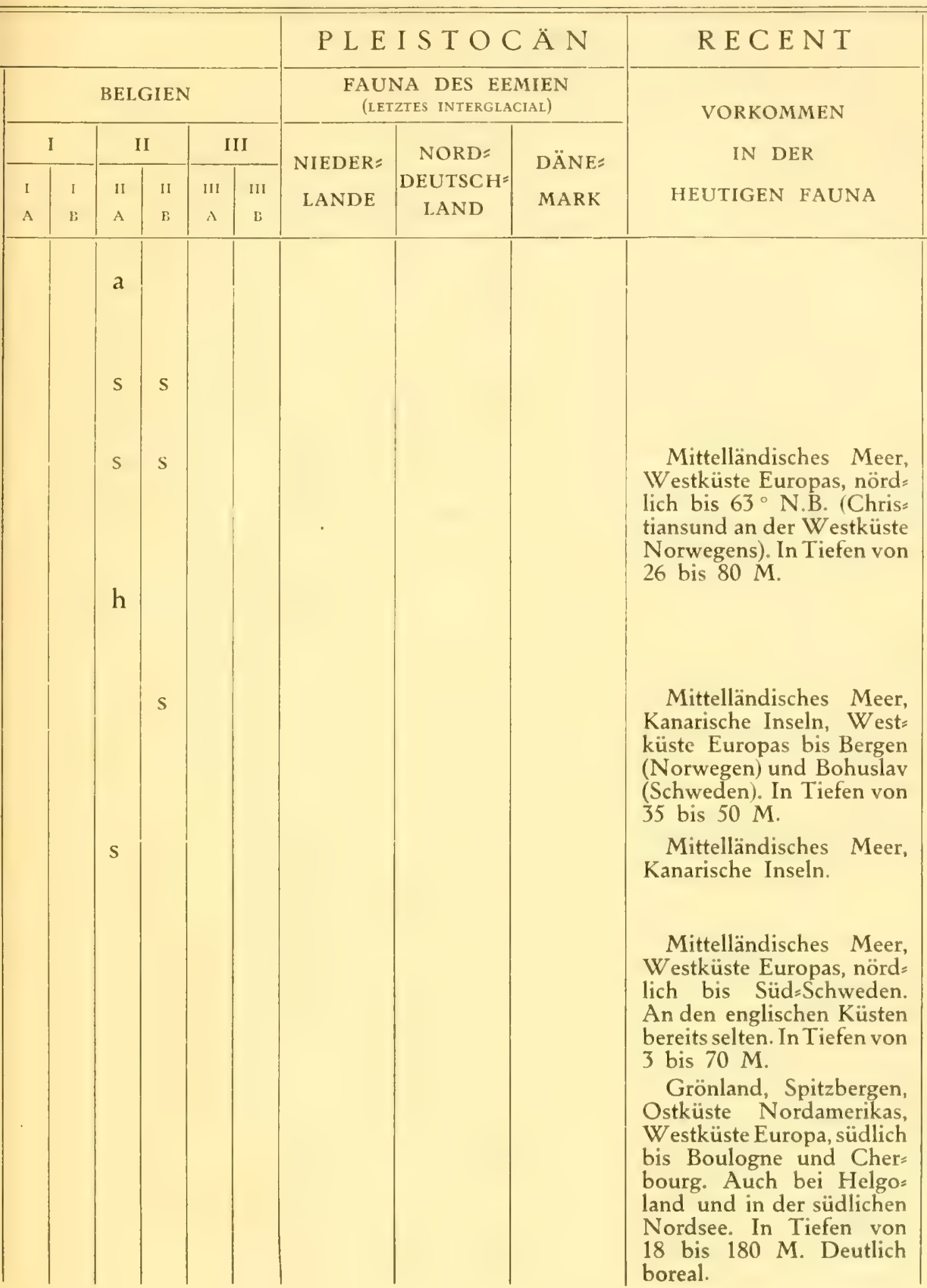

BEMERKUNGEN

Ziemlich häufig in IIa der Gegend Grave=Oss. In II a Belgiens nur spo= radisch aufgefunden.

Sehr selten in II a der Gegend Grave=Oss. In England unbekannt.

In II a der Gegend Grave= Oss selten. Nach Lorié auch aus IIIa (Gorkum) bekannt.

Ziemlich häufig in II a der Gegend Grave=Oss, in II a Belgiens nicht selten.

Sehr selten in II b der Gegend Grave=Oss. Auch in England und Belgien überall selten.

Sehr selten in II a der Gegend Grave=Oss. Auch in England und Belgien überall sehr selten.

Selten in II a der Gegend Grave=Oss. Im Pliocän Belgiens unbekannt.

Nach Lorié aus III a (Amsterdam, Utrecht) be= kannt. Von mir auch in III a (Oudewater, Baren. drecht) aufgefunden. 
$\mathrm{h}=$,sehr häufig" bis ,nicht selten".

$\mathrm{S}=$ "selten" bis "sehr selten".

\begin{tabular}{|c|c|c|c|c|c|c|c|c|c|c|c|c|c|}
\hline & & \multicolumn{12}{|c|}{ P L I OC $\ddot{A} \mathrm{~N}$} \\
\hline & & \multicolumn{6}{|c|}{ NIEDERLANDE } & \multicolumn{6}{|c|}{ ENGLAND } \\
\hline & & \multicolumn{2}{|c|}{ I } & \multicolumn{2}{|c|}{ II } & \multicolumn{2}{|c|}{ III } & \multicolumn{2}{|c|}{ I } & \multicolumn{2}{|c|}{ II } & \multicolumn{2}{|c|}{ III } \\
\hline & & $\begin{array}{l}\mathrm{I} \\
\mathrm{A}\end{array}$ & $\begin{array}{l}1 \\
\text { B }\end{array}$ & $\begin{array}{l}\text { II } \\
\text { A }\end{array}$ & $\begin{array}{l}\text { II } \\
\text { B }\end{array}$ & $\begin{array}{c}\text { III } \\
\text { A }\end{array}$ & $\begin{array}{l}\text { III } \\
\text { B }\end{array}$ & $\begin{array}{l}\mathrm{I} \\
\mathrm{A}\end{array}$ & $\begin{array}{l}1 \\
\text { B }\end{array}$ & $\begin{array}{l}\text { Il } \\
\text { A }\end{array}$ & $\begin{array}{l}\text { II } \\
\text { B }\end{array}$ & $\begin{array}{c}\text { III } \\
\text { A }\end{array}$ & $\begin{array}{l}\text { III } \\
\text { B }\end{array}$ \\
\hline 235 & $\begin{array}{l}\text { Pleurotoma cancellata Sow. } 1827 . \text {. . } \\
\text { (Murex cancellatus Sow. } \\
\text { Defrancia reticulata Renier). }\end{array}$ & & a & & $\mathrm{a}$ & & & & s & s & s & s & \\
\hline 236 & Pleurotoma clathrata M. de Serres . . & & & & & a & & & & & & & \\
\hline 237 & $\begin{array}{l}\text { Drillia crassa Bell } 1871 \text {. . . . . } \\
\quad \text { (Pleurotoma incrassata Dujard. 1837). }\end{array}$ & & & .5 & & & & & s & s & s & $s$ & \\
\hline 238 & Hadropleura Delheidi Vinc. 1890 . . . & & & & s & & & & & & & & \\
\hline 239 & Conus Dujardini Desh. 1830 & & & $\mathrm{r}$ & & & & & & & & & \\
\hline 240 & $\begin{array}{l}\text { Ringicula buccinea Broc. } 1814 \text { (Desh. 1830) } \\
\text { (Ringicula auriculata Philippi 1844). } \\
\text { (Marginella auriculata Ménard). }\end{array}$ & & a & $\mathrm{s}$ & $s$ & s & & s & h & h & $\mathrm{h}$ & $h=s$ & \\
\hline 241 & Ringicula ventricosa Sow. 1825 . . . . & & & $\mathrm{h}$ & $\mathrm{h}$ & $\mathrm{h}$ & & $\mathrm{h}$ & $\mathrm{h}$ & $\mathrm{s}$ & & $\mathrm{h}$ & h \\
\hline
\end{tabular}


$r=$ auf secundärer Lagerstätte.

$\mathrm{a}=$ anwesend, jedoch unbekannt ob häufig oder selten.

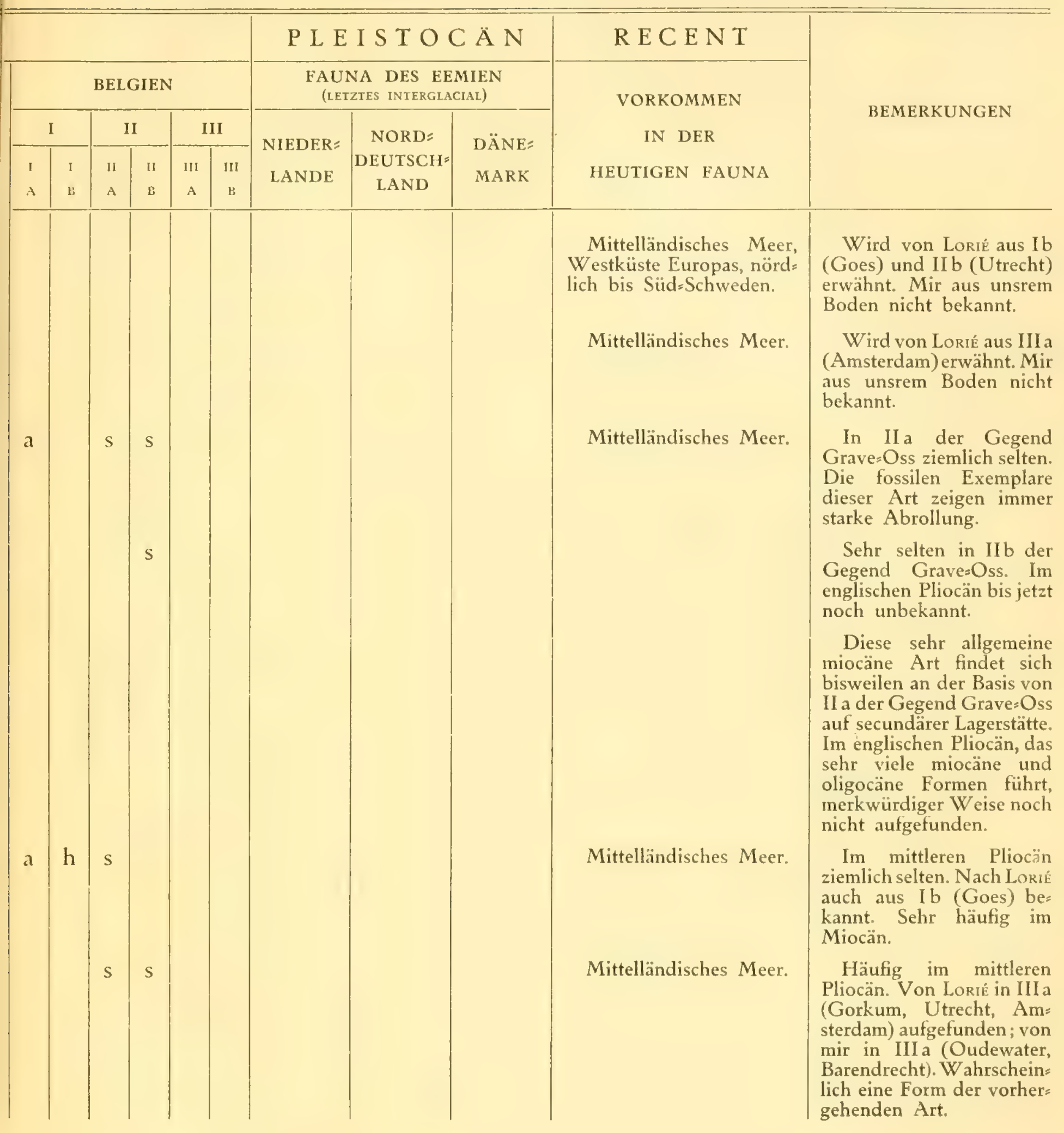


$\mathrm{h}=$ „sehr häufig" bis ,nicht selten".

$\mathrm{s}=$ „selten" bis „sehr selten".

Tornatella tornatilis Linn. 1766. (Actaeon tornatilis Linn.)

Cylichna cilindracea Penn. 1776 .

(Bulla convoluta Broc. 1814 (Sow. 1824)

Cylichna umbilicata Mont. 1808

(Bulla conulus Wood 1848).

(Bulla conuloidea Wood 1874).

(Tornatina umbilicata Mont.)

(Bulla truncatula Philippi 1844).

Bulla acuminata Brug. 1789.

(Cylichna acuminata Lovén 1846).

(Volvula acuminata Brug.)

Bulla utricula Broc. 1814

(Atys utricula Broc.)

Scaphander lignarius Linn. 1766

Conovulus pyramidalis Sow. 1822 (Melampus pyramidalis Sow).

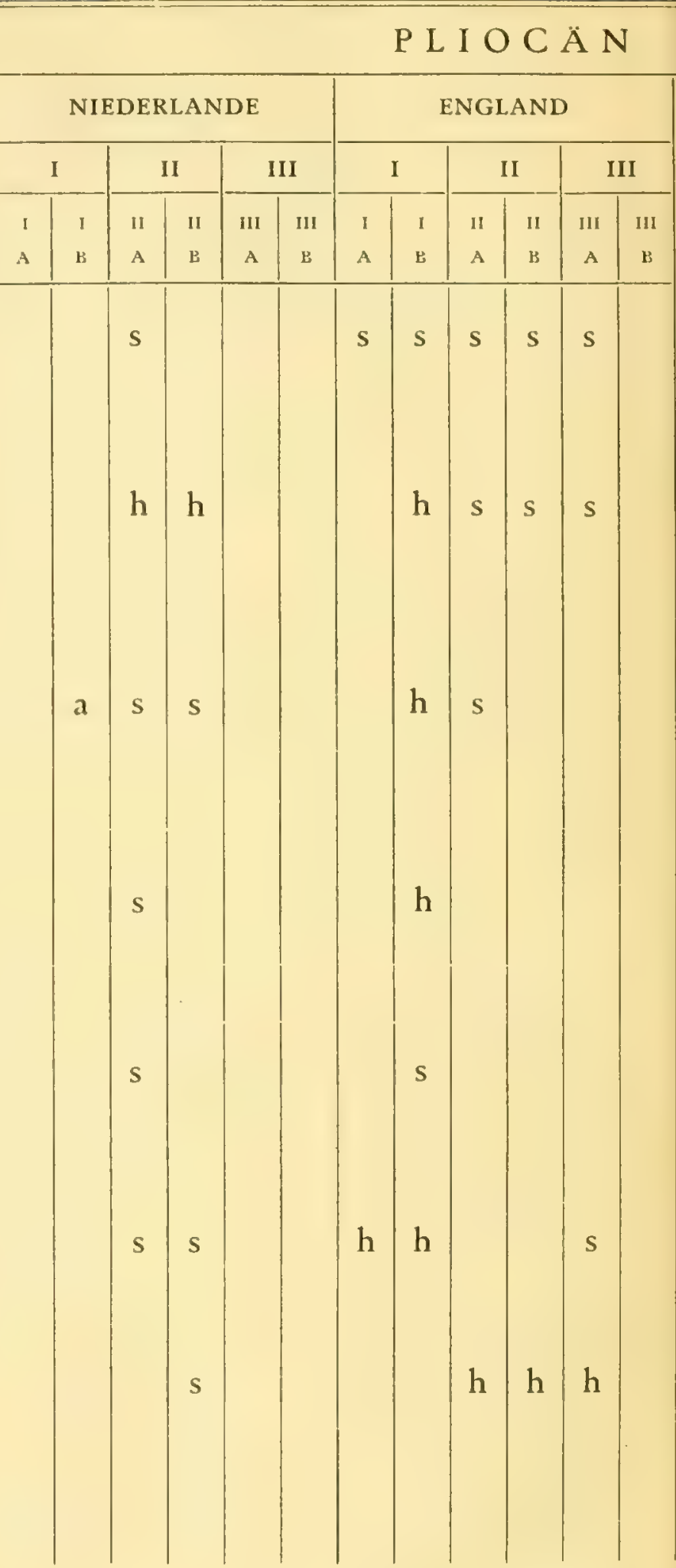


$\mathrm{r}=$ auf secundärer Lagerstätte.

$a=$ anwesend, jedoch unbekannt ob häufig oder selten.

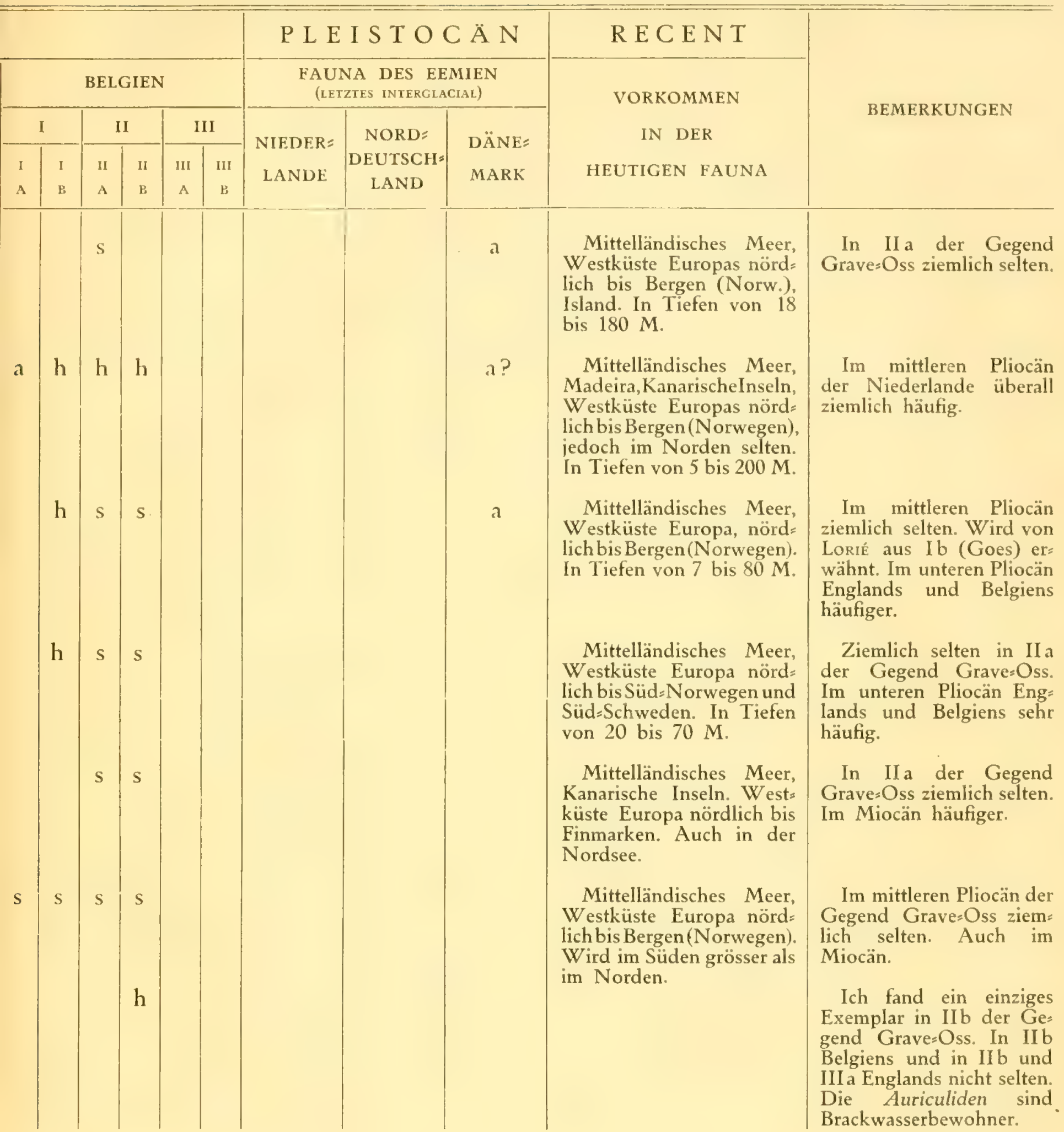




\section{LITERATUR ÜBER DAS MARINE PLIOCÄN IM NIEDERLÄN= DISCHEN BODEN(IN CHRONOLOGISCHER ANORDNUNG).}

1. P. HARTING.

De Bodem onder Gorinchem, onderzocht en beschreven.

Verhandelingen der Commissie voor de geologische beschrijving en kaart van. Nederland, Deel I, Haarlem, 1853.

2. W.C. H.STARING. De Bodem van Nederland. Tweede deel, Haarlem, 1860.

3. F. SEELHEIM. De grondboringen in Zeeland.

Verhandelingen der Kon. Akademie van Wetenschappen, Deel XIX, Amsterdam, 1879.

4. J. LORIÉ.

Résultats géologiques et paléontologiques des forages de puits à Utrecht, Goes et Gorkum (Contributions à la géologie des Pays=Bas I). Archives du Musée Teyler, Série II, vol. II, Haarlem, 1885.

5. J. LORIÉ. Les deux derniers forages d'Amsterdam (Contributions à la géologie des Pays:Bas IV).

Bulletin de la Société belge de géologie, tome III, Bruxelles, 1889.

6. F. W. HARMER.

On the pliocene deposits of Holland and their relation to the english and belgian crags, with a suggestion for the establishment of a new zone "Amstelien" and some remarks on the geographical conditions of the pliocene epoch in nothern Europe.

Quart. Journal of the geological Soc. of London, vol. LII, London, 1896.

7. F. W. HARMER.

Les dépôts tertiaires supérieures du bassin anglo:belge.

Bulletin de la Soc. belge de géologie, tome X, Bruxelles, 1896.

8. J. LORIÉ Sondages en Zélande et en Brabant (Contributions à la géologie des Pays: Bas X).

Bulletin de la Société belge de géologie, tome XVII, Bruxelles, 1903.

9. J. LORIÉ.

De geologische bouw der Geldersche Vallei, benevens beschrijving van eenige nieuwe grondboringen VII.

Mededeelingen omtrent de geologie van Nederland, verzameld door de commissie voor het geologisch onderzoek, No. 35.

Verhandelingen der Kon. Academie van Wetenschappen (Tweede Sectie), Deel XIII, No. 1, Amsterdam, 1906.

10. P. TESCH

Over pleistoceen en plioceen in den Nederlandschen bodem. I.

Tijdschrift van het Kon. Ned. Aardrijkskundig Genootschap, Tweede Serie, Deel XXVII, aflevering 6, Leiden, 1910.

11. P. TESCH. Over een voorkomen van middenspliocene lagen aan de oppervlakte in Zeeuwsch=Vlaanderen.

Tijdschrift van het Kon. Ned. Aardrijkskundig Genootschap, Tweede Serie, deel XXVIII, aflevering I, Leiden, 1911.

12. P. TESCH.

Over pleistoceen en plioceen in den Nederlandschen Bodem. II.

Tijdschrift van het Kon. Ned. Aardrijkskundig Genootschap, Tweede Serie, deel XXVIII, aflevering 4, Leiden, 1911. 



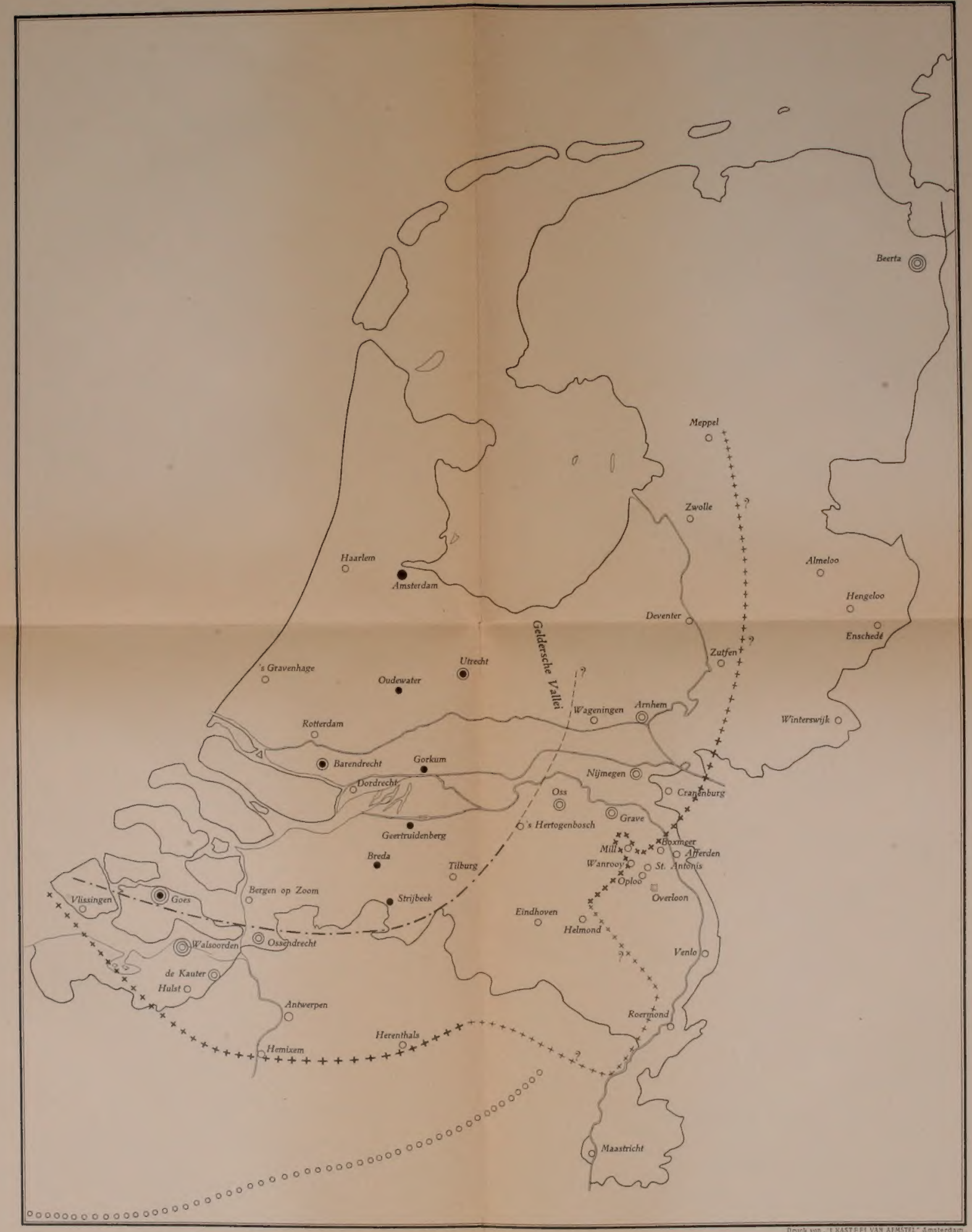

-... - . - Südliche und östliche Grenze des marinen oberen Pliocäns.

Sưdliche und östliche Grenze des marinen mittleren Pliocäns.
00000000

Südliche Grenze des maninen unteren Pliocïn in Belgien (nach HАлмеи und Rutor).
- Stellen wo das marine obere Pliocän erbohrt wurde.

(C) " . " . " mittlere " . "

(C) . " . untere . . . 


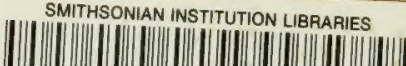

39088007545296

的

DRUCK VON

'T KASTEEL VAN

AEMSTEL

AMSTERDAM 